\title{
Conjugation of Nucleosides and Oligonucleotides by [3+2] Cycloaddition
}

Anup M. Jawalekar, Nico Meeuwenoord, J. (Sjef) G. O. Cremers, Herman S. Overkleeft, Gijs A. van der Marel, Floris P. J. T. Rutjes, Floris L. van Delft*

\section{Supporting information}

\section{Contents}

General methods and materials

HPLC and HRMS-data of oligonucleotides 5, 6, 7 S18-S20

${ }^{1} \mathrm{H}$ and ${ }^{13} \mathrm{C}$ NMR spectra of triazole products of Table $1+2$ S21-66

\section{General methods and materials}

Pyridine was dried over molecular sieves $4 \AA$. Commercially available reagents were used as received. Dichloromethane and DMF were freshly distilled from calcium hydride. Air and moisture sensitive reactions were carried out under an inert atmosphere of dry argon. Column chromatography was performed using across silica gel $(0.035-0.070 \mathrm{~mm})$, TLC was performed with precoated silica gel plates. ${ }^{1} \mathrm{H}$ NMR and ${ }^{13} \mathrm{C}$ NMR spectra are reported in units of ppm on the $\delta$ scale. Oligonucleotides were synthesised on universal support with commercially available phosphoramidites and the prepared phosphoamidites $\mathbf{4 a}$ and $\mathbf{4 b}$. MALDI-TOF mass spectrometry was executed in the linear positive mode with a matrix of HPA (3-hydroxypicolinic acid) and ammonium citrate. Melting points are uncorrected. 5-Chloro-1-pentyne, benzyl azide, propargyl alcohol, 1,7-octadiyne and 2',3'-O-isopropylideneadenosine were used as received. 1-Chloro-3-azidopropane, ${ }^{1}$ 2,3,4,6-tetra- $O$-acetyl- $\beta$-D-glucopyranosyl azide, ${ }^{2}, 5^{\prime}$ azido-5'-deoxyadenosine ${ }^{3}, 2^{\prime}$-azido-2'-deoxyuridine ${ }^{4}$, 4-azido-2,2,6,6-tetramethylpiperidine 1-oxyl ${ }^{5}, 7-(1$ propyn)oxycoumarin $^{6},(E)-4-\left[4-\left(\left(\right.\right.\right.$ dimethylamino)phenyl)-diazenyl]- $N$-(prop-2-ynyl)benzamide ${ }^{7}$ and $2-O$ methyladenosine ${ }^{8}$ were prepared according to known procedures. 


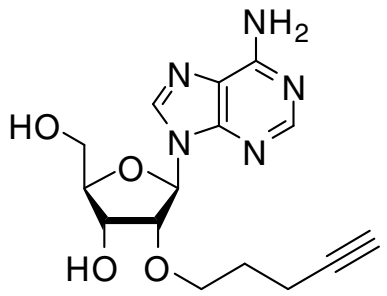

$2 a$

Adenosine (5 g, $18.72 \mathrm{mmol}$ ) was dissolved in hot anhydrous DMF (250 mL) under an inert atmosphere. The solution was cooled to $5{ }^{\circ} \mathrm{C}$, and $\mathrm{NaH}(1 \mathrm{~g}, 25 \mathrm{mmol}, 60 \%$ dispersion in mineral oil) was subsequently added, followed by the addition of TBAI (1.5 g, $4.06 \mathrm{mmol})$ and 5-chloro-1-pentyne (2.5 $\mathrm{mL}, 23.84 \mathrm{mmol})$. The reaction mixture was allowed to stir for 3 days at $55^{\circ} \mathrm{C}$. Evaporation of the solvent under reduced pressure resulted in a suspension, which was absorbed on silica gel. Flash-chromatography $\left(\mathrm{CH}_{2} \mathrm{Cl}_{2} / \mathrm{MeOH}, 95: 5\right)$, gave a mixture of 2'-O and 3'-O-alkynylated products. Selective crystallization from anhydrous ethanol provided the title compound $\mathbf{2 a}(2.5 \mathrm{~g}, 40 \%)$ as colorless needles. ${ }^{1} \mathrm{H}$ NMR (300 MHz, DMSO) $\delta 8.36(\mathrm{~s}, 1 \mathrm{H}), 8.12(\mathrm{~s}, 1 \mathrm{H}), 7.33$ (br s, 2H), $6.00(\mathrm{~d}, J=6 \mathrm{~Hz}, 1 \mathrm{H}), 5.39(\mathrm{~m}, 1 \mathrm{H}), 5.17(\mathrm{~d}, J$ $=5.4 \mathrm{~Hz}, 1 \mathrm{H}), 4.46(\mathrm{~m}, 1 \mathrm{H}), 4.29(\mathrm{~m}, 1 \mathrm{H}), 3.97(\mathrm{~m}, 1 \mathrm{H}), 3.64-3.32(\mathrm{~m}, 4 \mathrm{H}), 2.64(\mathrm{~m}, 1 \mathrm{H}), 2.08(\mathrm{~m}, 2 \mathrm{H})$, 1.58 (m, 2H). ${ }^{13} \mathrm{C}$ NMR (75 MHz, DMSO) $\delta$ 156.1, 152.5, 149.0, 139.7, 119.3, 86.3, 86.0, 83.9, 81.0, 71.1, 69.0, 68.3, 61.4, 28.1, 14.3.HRMS calcd for $\mathrm{C}_{15} \mathrm{H}_{19} \mathrm{~N}_{5} \mathrm{O}_{4}\left(\mathrm{M}+\mathrm{Na}^{+}\right)$356.1335, found 356.1308.

\section{2'-O-(1-Pentyn-5-yl)-6- $N$-benzoyladenosine (3a)}

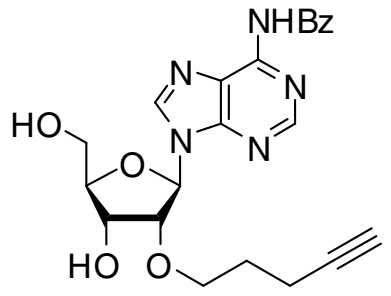

$3 a$

2'-O-(1-Pentyn-5-yl)adenosine (2a) (200 mg, $0.6 \mathrm{mmol})$ was coevaporated twice with anhydrous pyridine and dissolved in pyridine $(4 \mathrm{~mL})$ followed by the addition of trimethylsilyl chloride $(0.4 \mathrm{~mL}, 3.2 \mathrm{mmol})$. After $30 \mathrm{~min}$, benzoyl chloride $(0.1 \mathrm{~mL}, 0.8 \mathrm{mmol})$ was added and stirring was continued for $3 \mathrm{~h}$. The reaction mixture was cooled to $0{ }^{\circ} \mathrm{C}$ and diluted with $\mathrm{H}_{2} \mathrm{O}(1.5 \mathrm{~mL})$. Subsequently, the reaction mixture was treated with an aqueous concentrated $\mathrm{NH}_{3}$-solution $(2 \mathrm{~mL})$ and stirred for $30 \mathrm{~min}$ at room temperature. The mixture was evaporated to dryness and purified chromatographically with $\left(\mathrm{CH}_{2} \mathrm{Cl}_{2}\right.$ /Acetone, 95:5) followed by $\mathrm{CH}_{2} \mathrm{Cl}_{2} / \mathrm{MeOH}$ (98:2) yielding compound 3a (240 $\left.\mathrm{mg}, 92 \%\right)$ as a colorless foam. ${ }^{1} \mathrm{H}$ NMR (300 MHz, DMSO) $\delta 11.18(\mathrm{~s}, 1 \mathrm{H}), 8.74(\mathrm{~s}, 1 \mathrm{H}), 8.73(\mathrm{~s}, 1 \mathrm{H}), 8.04-7.51$ (m, $5 \mathrm{H}), 6.15(\mathrm{~d}, J=5.1 \mathrm{~Hz}, 1 \mathrm{H}), 5.23(\mathrm{~m}, 1 \mathrm{H}), 5.13(\mathrm{~m}, 1 \mathrm{H}), 4.49(\mathrm{~m}, 1 \mathrm{H}), 4.33(\mathrm{~m}, 1 \mathrm{H}), 3.99(\mathrm{~m}, 1 \mathrm{H}), 3.66-$ 
$3.50(\mathrm{~m}, 4 \mathrm{H}), 2.65(\mathrm{~m}, 1 \mathrm{H}), 2.12(\mathrm{~m}, 2 \mathrm{H}), 1.65(\mathrm{~m}, 2 \mathrm{H}) .{ }^{13} \mathrm{C}$ NMR (75 MHz, DMSO) $\delta$ 165.6, 151.7, 150.4, 142.9, 133.3, 132.4, 128.4, 128.2, 127.4, 125.8, 86.0, 85.8, 83.8, 81.1, 71.2, 68.8, 68.4, 61.0, 28.1, 14.3. HRMS calcd for $\mathrm{C}_{22} \mathrm{H}_{23} \mathrm{~N}_{5} \mathrm{O}_{5}\left(\mathrm{M}+\mathrm{Na}^{+}\right) 460.1597$ found 460.1571 .

$5^{\prime}-O$-(4,4'-Dimethoxytriphenylmethyl)-2'-O-(1-pentyn-5-yl)-6- $N$-benzoyladenosine

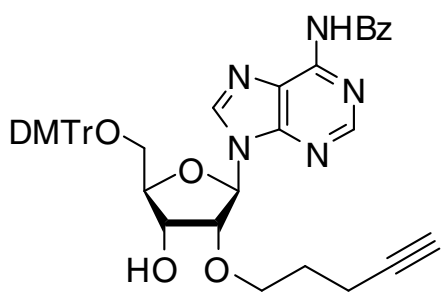

2'-O-(1-Pentyn-5-yl)-6- $N$-benzoyladenosine (3a) $(180 \mathrm{mg}, 0.41 \mathrm{mmol})$ was coevaporated twice with anhydrous pyridine and dissolved in pyridine $(4 \mathrm{~mL})$, followed by the addition of DMTrCl (200 mg, 0.59 $\mathrm{mmol}$ ). The mixture was stirred for $3 \mathrm{~h}$ at room temperature and quenched by the addition of a 5\% aqueous $\mathrm{NaHCO}_{3}$ solution $(10 \mathrm{~mL})$. The aqueous layer was extracted with $\mathrm{CH}_{2} \mathrm{Cl}_{2}(3 \mathrm{x} 15 \mathrm{~mL})$. The combined organic layers were dried over $\mathrm{Na}_{2} \mathrm{SO}_{4}$ and concentrated in vacuo. The residue was subjected to column chromatography (acetone/ $\left.\mathrm{CH}_{2} \mathrm{Cl}_{2}, 5: 95\right)$ and furnished the title compound $(270 \mathrm{mg}, 89 \%)$ as a colorless foam. ${ }^{1} \mathrm{H}$ NMR (300 MHz, DMSO) $\delta 11.20$ (s, 1H), 8.67 (s, 1H), 8.59 (s, 1H), 8.04-6.81 (m, $18 \mathrm{H}), 6.17(\mathrm{~d}, J=4.8 \mathrm{~Hz}, 1 \mathrm{H}), 5.27(\mathrm{~d}, J=6.3,1 \mathrm{H}), 4.67(\mathrm{~m}, 1 \mathrm{H}), 4.44(\mathrm{~m}, 1 \mathrm{H}), 4.12(\mathrm{~m}, 1 \mathrm{H}), 3.70(\mathrm{~s}$, $6 \mathrm{H}), 3.68-3.55(\mathrm{~m}, 2 \mathrm{H}), 3.25$ (bs, 2H), $2.67(\mathrm{~d}, 1 \mathrm{H}), 2.15(\mathrm{~m}, 2 \mathrm{H}), 1.66(\mathrm{~m}, 2 \mathrm{H}) .{ }^{13} \mathrm{C}$ NMR $(75 \mathrm{MHz}$, DMSO) $\delta 165.6,158.0,152.0,151.7,150.5,144.8,143.2,135.6,135.4,133.3,132.4,129.7,128.5,128.4$, $127.8,127.7,126.6,125.9,113.1,86.3,85.5,83.9,83.6,80.2,71.2,69.2,68.6,63.5,55.0,28.1,14.4$. HRMS calcd for $\mathrm{C}_{43} \mathrm{H}_{42} \mathrm{~N}_{5} \mathrm{O}_{7}\left(\mathrm{M}+\mathrm{H}^{+}\right) 740.3084$ found 740.3062 .

5'-O-(4,4'-Dimethoxytriphenylmethyl)-2' - $O$-(1-pentyn-5-yl)-6- $N$-benzoyladenosine-3'- $O$ (2-cyanoethyl $N, N$-diisopropylphosphoramidite) (4a)

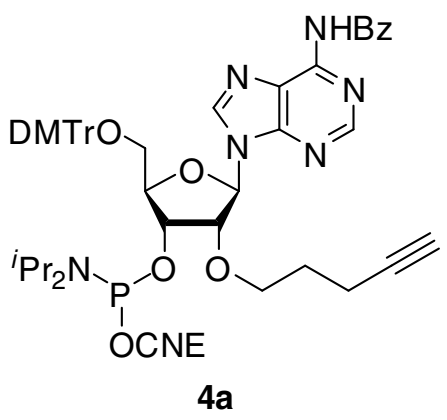

To a solution of 5'-O-(4,4'-dimethoxytriphenylmethyl)-2'-O-(1-pentyn-5-yl)-6- $N$-benzoyladenosine (90 $\mathrm{mg}, 0.12 \mathrm{mmol})$ in dry $\mathrm{CH}_{2} \mathrm{Cl}_{2}(2 \mathrm{~mL})$ was subsequently added, DIPEA $(35 \mu \mathrm{L}, 0.134 \mu \mathrm{mol})$ and 2-cyanoethyl $N, N$-diisopropylphosphoramidochloridite $(30 \mu \mathrm{L}, 0.134 \mu \mathrm{mol})$. After stirring for $80 \mathrm{~min}$ 
under an inert atmosphere, the reaction mixture was diluted with $\mathrm{CH}_{2} \mathrm{Cl}_{2}(15 \mathrm{~mL})$ and a $5 \%$ aqueous $\mathrm{NaHCO}_{3}$ solution $(5 \mathrm{~mL})$. The mixture was extracted with $\mathrm{CH}_{2} \mathrm{Cl}_{2}(2 \times 10 \mathrm{~mL})$, the combined organic layers were dried over $\mathrm{Na}_{2} \mathrm{SO}_{4}$, filtered and concentrated in vacuo. The residue purified by flash chromatography (acetone/ $\left.\mathrm{CH}_{2} \mathrm{Cl}_{2}, 5: 95\right)$ to give $\mathbf{4 a}(110 \mathrm{mg}, 92 \%)$ as a colorless foam. ${ }^{31} \mathrm{P}$ NMR (121 $\mathrm{MHz} \mathrm{CDCl}_{3} 150.1,149.6 .{ }^{1} \mathrm{H} \mathrm{NMR}(300 \mathrm{MHz}, \mathrm{DMSO}) \delta 8.65(\mathrm{~s}, 1 \mathrm{H}), 8.64(\mathrm{~s}, 1 \mathrm{H}), 8.05$ (d, J=7.2 Hz, 2H), $7.64(\mathrm{t}, J=7.2 \mathrm{~Hz}, 1 \mathrm{H}), 7.55(\mathrm{t}, J=7.6 \mathrm{~Hz}, 2 \mathrm{H}), 7.38-7.35(\mathrm{~m}, 2 \mathrm{H}), 7.25-7.21(\mathrm{~m}, 7 \mathrm{H}), 6.85-6.81(\mathrm{~m}$, $4 \mathrm{H}), 6.18(\mathrm{dd}, J=14.0 \mathrm{~Hz}, J=5.2 \mathrm{~Hz}, 1 \mathrm{H}), 4.92(\mathrm{dd}, J=14.8 \mathrm{~Hz}, J=5.2 \mathrm{~Hz}, 1 \mathrm{H}), 4.72-4.67$ (m, 1H), 4.30$4.22(\mathrm{~m}, 1 \mathrm{H}), 3.89-3.78(\mathrm{~m}, 1 \mathrm{H}), 3.74-3.53(\mathrm{~m}, 10 \mathrm{H}), 3.40-3.24(\mathrm{~m}, 3 \mathrm{H}), 2.79(\mathrm{t}, J=6.4 \mathrm{~Hz}, 1.2 \mathrm{H}), 2.66$ $(\mathrm{dd}, J=7.2 \mathrm{~Hz}, J=2.8 \mathrm{~Hz}, 1 \mathrm{H}), 2.6(\mathrm{t}, J=6.4 \mathrm{~Hz}, 0.78 \mathrm{H}), 2.15-2.10(\mathrm{~m}, 2 \mathrm{H}), 1.15(\mathrm{t}, J=6.4 \mathrm{~Hz}, 8 \mathrm{H}), 1.04$ $(\mathrm{d}, J=6.8 \mathrm{~Hz}, 4 \mathrm{H}) .{ }^{13} \mathrm{C} \mathrm{NMR}\left(75 \mathrm{MHz}, \mathrm{CD}_{2} \mathrm{Cl}_{2}\right) \delta 163.9,158.2,151.8,151.1,144.2,141.6,141.5,135.2$, 135.1, 133.6, 132.1, 129.6, 128.3, 127.7, 127.4, 127.3, 126.4, 112.6, 87.0, 86.9, 86.1, 86.0, 83.2, 83.0, 80.0, 70.9, 70.7, 70.6, 70.4, 68.9, 68.7, 67.9, 62.6, 62.3, 58.3, 58.0, 57.7, 54.7, 42.9, 42.8, 42.6, 28.0, 23.9,20.0, 19.9, 19.7, 14.4, 14.3. HRMS calcd for $\mathrm{C}_{52} \mathrm{H}_{59} \mathrm{~N}_{7} \mathrm{O}_{8} \mathrm{P}\left(\mathrm{M}+\mathrm{H}^{+}\right)$940.4163 found 940.4127.

\section{2'-O-(3-Azidoprop-1-yl)adenosine (2b)}

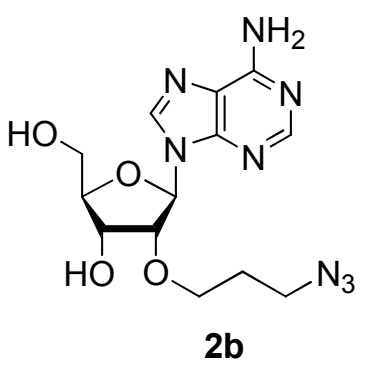

Adenosine (7.5 g, $28.06 \mathrm{mmol})$ was dissolved in hot anhydrous DMF (300 mL) under an inert atmosphere and cooled to $5{ }^{\circ} \mathrm{C}$. Subsequently $\mathrm{NaH}(1.5 \mathrm{~g}, 37.5 \mathrm{mmol}, 60 \%$ dispersion in mineral oil $)$ was added. The reaction mixture was stirred at ambient temperature for $5 \mathrm{~min}$ followed by the addition of TBAI ( $2.25 \mathrm{~g}$, $6.1 \mathrm{mmol})$ and 1-chloro-3-azidopropane $(3.5 \mathrm{~g}, 29.28 \mathrm{mmol})$. The mixture was stirred for 3 days at $50{ }^{\circ} \mathrm{C}$ Evaporation of the solvent under reduced pressure resulted in a suspension, which was absorbed on silica gel. Flash chromatography $\left(\mathrm{CH}_{2} \mathrm{Cl}_{2} / \mathrm{MeOH}, 95: 5\right)$, gave a mixture of 2'-O and 3'-O-alkynylated products. Selective crystallization from anhydrous ethanol provided the title compound $\mathbf{2 b}(3.0 \mathrm{~g}, 31 \%)$ as colorless needles. ${ }^{1} \mathrm{H}$ NMR (300 MHz, DMSO) $\delta 8.36(\mathrm{~s}, 1 \mathrm{H}), 8.12(\mathrm{~s}, 1 \mathrm{H}), 7.32(\mathrm{bs}, 2 \mathrm{H}), 6.00(\mathrm{~d}, J=6 \mathrm{~Hz}, 1 \mathrm{H})$, $5.38(\mathrm{~m}, 1 \mathrm{H}), 5.20(\mathrm{~d}, J=5.4 \mathrm{~Hz}, 1 \mathrm{H}), 4.46(\mathrm{~m}, 1 \mathrm{H}), 4.29(\mathrm{~m}, 1 \mathrm{H}), 3.97(\mathrm{~m}, 1 \mathrm{H}), 3.64-3.43(\mathrm{~m}, 4 \mathrm{H}), 3.27$ (m, 2H), 1.67 (m, 2H). ${ }^{13} \mathrm{C}$ NMR (75 MHz, DMSO) $\delta 156.1,152.5,149.0,139.6,119.3,86.3,86.0,81.0$, 68.0, 66.7, 61.4, 47.6, 28.5. HRMS calcd for $\mathrm{C}_{13} \mathrm{H}_{18} \mathrm{~N}_{8} \mathrm{O}_{4}\left(\mathrm{M}+\mathrm{Na}^{+}\right) 351.1524$ found 351.1525 .

\section{2'-O-(3-Azidoprop-1-yl)-6- $N$-benzoyladenosine (3b)}




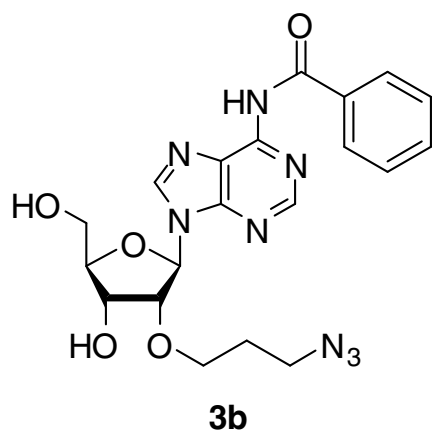

2'-O-(3-Azidoprop-1-yl)adenosine (2b) $(200 \mathrm{mg}, 0.44 \mathrm{mmol})$ was coevaporated twice with anhydrous pyridine and dissolved in pyridine $(4 \mathrm{~mL})$, followed by the addition of trimethylsilyl chloride $(0.3 \mathrm{~mL}$, $2.34 \mathrm{mmol})$. After $30 \mathrm{~min}$, benzoyl chloride $(74 \mu \mathrm{L}, 0.58 \mathrm{mmol})$ was added and stirring was continued for $3 \mathrm{~h}$. The reaction mixture was cooled to $0{ }^{\circ} \mathrm{C}$ and diluted with $\mathrm{H}_{2} \mathrm{O}(1.5 \mathrm{~mL})$. Subsequently, the reaction mixture was treated with an aqueous concentrated $\mathrm{NH}_{3}$-solution $(2 \mathrm{~mL})$ and stirred for 30 min at room temperature. The mixture was evaporated to dryness and purified chromatographically with $\left(\mathrm{CH}_{2} \mathrm{Cl}_{2}\right.$ /acetone, 95:5) followed by $\mathrm{CH}_{2} \mathrm{Cl}_{2} / \mathrm{MeOH}$ (98:2) yielding compound $\mathbf{3 b}(176 \mathrm{mg}, 92 \%)$ as a colorless foam. ${ }^{1} \mathrm{H}$ NMR (300 MHz, DMSO) $\delta 11.18$ (s, 1H), $8.74(\mathrm{~s}, 1 \mathrm{H}), 8.73(\mathrm{~s}, 1 \mathrm{H})$, 8.04-7.51 (m, $5 \mathrm{H}), 6.15(\mathrm{~d}, J=5.1 \mathrm{~Hz}, 1 \mathrm{H}), 5.23(\mathrm{~m}, 1 \mathrm{H}), 5.13(\mathrm{~m}, 1 \mathrm{H}), 4.49(\mathrm{~m}, 1 \mathrm{H}), 4.33(\mathrm{~m}, 1 \mathrm{H}), 3.99(\mathrm{~m}, 1 \mathrm{H}), 3.66-$ $3.50(\mathrm{~m}, 4 \mathrm{H}), 2.65(\mathrm{~m}, 1 \mathrm{H}), 2.12(\mathrm{~m}, 2 \mathrm{H}), 1.65(\mathrm{~m}, 2 \mathrm{H}) .{ }^{13} \mathrm{C}$ NMR $(75 \mathrm{MHz}, \mathrm{DMSO}) \delta$ 165.6, 151.7, 150.4, 142.9, 133.3, 132.4, 128.4, 128.2, 127.4, 125.8, 86.0, 85.8, 83.8, 81.1, 71.2, 68.8, 68.4, 61.0, 28.1, 14.3. HRMS calcd for $\mathrm{C}_{22} \mathrm{H}_{23} \mathrm{~N}_{5} \mathrm{O}_{6}\left(\mathrm{M}+\mathrm{H}^{+}\right) 455.1791$ found 455.1807 .

\section{5 '-O-(4,4'-Dimethoxytriphenylmethyl)-2'-O-(3-azidoprop-1-yl)-6- $N$-benzoyladenosine}

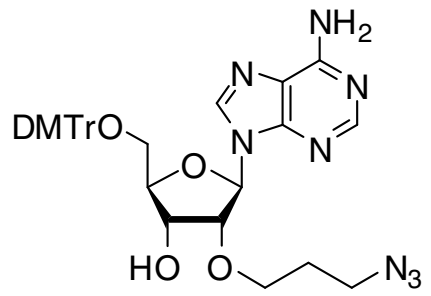

2'-O-(3-Azidoprop-1-yl)-6- $N$-benzoyladenosine (3b) $(720 \mathrm{mg}, 1.58 \mathrm{mmol})$ was coevaporated twice with anhydrous pyridine and dissolved in pyridine $(16 \mathrm{~mL})$, followed by addition of DMTrCl $(720 \mathrm{mg}, 2.36$ mmol). The mixture was stirred for $3 \mathrm{~h}$ at room temperature and quenched by the addition of a $5 \%$ aqueous $\mathrm{NaHCO}_{3}$-solution $(40 \mathrm{~mL})$. The aqueous layer was extracted with $\mathrm{CH}_{2} \mathrm{Cl}_{2}(3 \mathrm{x} 60 \mathrm{~mL})$. The combined organic layers were dried over $\mathrm{Na}_{2} \mathrm{SO}_{4}$ and concentrated in vacuo. The residue was subjected to column chromatography (Acetone $\left./ \mathrm{CH}_{2} \mathrm{Cl}_{2}, 5: 95\right)$ and furnished the title compound $(1.1 \mathrm{~g}, 92 \%)$ as a colorless foam. ${ }^{1} \mathrm{H}$ NMR (300 MHz, DMSO) $\delta 11.21(\mathrm{~s}, 1 \mathrm{H}), 8.66(\mathrm{~s}, 1 \mathrm{H}), 8.60(\mathrm{~s}, 1 \mathrm{H}), 8.02(\mathrm{~d}, J=6.9$ $\mathrm{Hz}, 2 \mathrm{H}), 7.65-7.52(\mathrm{~m}, 3 \mathrm{H}), 7.32-7.20(\mathrm{~m}, 9 \mathrm{H}), 6.83-6.80(\mathrm{~m}, 4 \mathrm{H}), 6.16$ (d, J=4.8 Hz), 5.27 (d, J=6.0 Hz, $1 \mathrm{H}), 4.63(\mathrm{t}, J=4.9 \mathrm{~Hz}, 1 \mathrm{H}), 4.44-4.42(\mathrm{~m}, 1 \mathrm{H}), 4.11-4.10(\mathrm{~m}, 1 \mathrm{H}), 3.69-3.54(\mathrm{~m}, 8 \mathrm{H}), 3.36-3.25(\mathrm{~m}, 2 \mathrm{H})$, $1.74(\mathrm{~m}, 2 \mathrm{H}) .{ }^{13} \mathrm{C}$ NMR (75 MHz, DMSO) $\delta 158.0,151.9,151.6,150.5,144.8,143.2,135.6,135.4,133.4$, 
$132.4,129.7,128.5,128.4,127.7,127.6,125.9,113.3,86.3,85.5,83.6,80.2,69.1,67.0,55.0,47.6,28.5$. HRMS calcd for $\mathrm{C}_{41} \mathrm{H}_{42} \mathrm{~N}_{8} \mathrm{O}_{7}\left(\mathrm{M}+\mathrm{H}^{+}\right) 757.3098$ found 757.3093 .

5'-O-(4,4'-Dimethoxytriphenylmethyl)-2'-O-(3-azidoprop-1-yl)-6- $N$-benzoyladenosine-3' $-O$ (2-cyanoethyl $N, N$-diisopropylphosphoramidite) (4b)

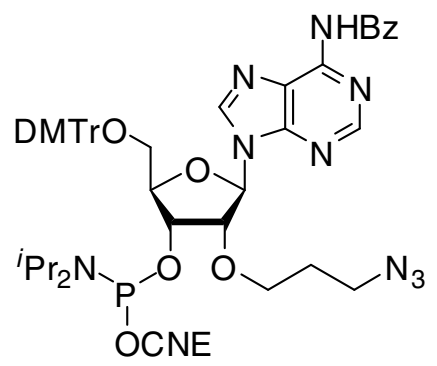

$4 b$

To a solution of 5'-O-(4,4'-dimethoxytriphenylmethyl)-2'-O-(3-azidoprop-1-yl)-6- $N$-benzoyladenosine (300 mg, $0.40 \mathrm{mmol}$ ) in dry $\mathrm{CH}_{2} \mathrm{Cl}_{2}$ ( $4 \mathrm{~mL}$ ) was subsequently added, DIPEA (140 $\left.\mu \mathrm{L}, 0.536 \mu \mathrm{mol}\right)$ and 2-cyanoethyl $N, N$-diisopropylphosphoramidochloridite $(120 \mu \mathrm{L}, 0.536 \mu \mathrm{mol})$. After stirring for $60 \mathrm{~min}$ under an inert atmosphere, the reaction mixture was diluted with $\mathrm{CH}_{2} \mathrm{Cl}_{2}(13 \mathrm{~mL})$ and a $5 \%$ aqueous $\mathrm{NaHCO}_{3}$ solution $(3 \mathrm{~mL})$. The mixture was rapidly extracted with $\mathrm{CH}_{2} \mathrm{Cl}_{2}(2 \times 10 \mathrm{~mL})$, the combined organic layers were dried over $\mathrm{Na}_{2} \mathrm{SO}_{4}$, filtered and concentrated in vacuo. The residue purified by flash chromatography (acetone $\left./ \mathrm{CH}_{2} \mathrm{Cl}_{2}, 5: 95\right)$ to give $\mathbf{4 b}(90 \mathrm{mg}, 19 \%)$ as a colorless foam. ${ }^{31} \mathrm{P}$ NMR (121 MHz, $\left.\mathrm{CDCl}_{3}\right)$ 153.9 153.3. 


\section{4-Chloromethyl-7-hydroxycoumarine}<smiles>O=c1cc(CCl)c2ccc(O)cc2o1</smiles>

According to literature procedure ${ }^{9}$. Quantities used: $60 \% \mathrm{H}_{2} \mathrm{SO}_{4}(50 \mathrm{~mL})$, resorcinol $(2.20 \mathrm{~g}, 20 \mathrm{mmol})$, cloro-ethyl-acetoacetate $(2.72 \mathrm{~mL}, 20 \mathrm{mmol})$.Yield $4.0 \mathrm{~g}(95 \%)$ as a light pink solid. The analytical data are in agreement with literature values. ${ }^{1} \mathrm{H}$ NMR (400 MHz, DMSO-d.6) $\delta$ ppm 10.64 (br s, 1H), 7.67 (d, $J$ $=8.8 \mathrm{~Hz}, 1 \mathrm{H}), 6.83(\mathrm{dd}, J=2.4,8.8 \mathrm{~Hz}, 1 \mathrm{H}), 6.74(\mathrm{~d}, J=2.3 \mathrm{~Hz}, 1 \mathrm{H}), 6.41(\mathrm{~s}, 1 \mathrm{H}), 4.94(\mathrm{~d}, J=0.7 \mathrm{~Hz}$, 2H). HRMS calcd for $\mathrm{C}_{10} \mathrm{H}_{7} \mathrm{ClO}_{3}[\mathrm{M}+\mathrm{H}]^{+} 211.0162$, found 211.0152 .

\section{4-Chloromethyl-7-methoxycoumarine}

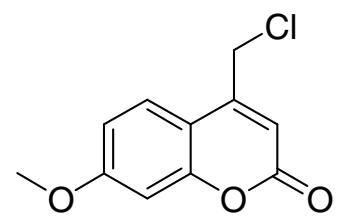

According to literature procedure. ${ }^{10}$ To a suspension of hydroxyl-chloro-coumarin $(1.43 \mathrm{~g}, 6.79 \mathrm{mmol})$ and $\mathrm{K}_{2} \mathrm{CO}_{3}(3.75 \mathrm{~g}, 27.2 \mathrm{mmol})$ in dry acetone $(70 \mathrm{~mL})$ was added $\mathrm{Me}_{2} \mathrm{SO}_{4}(0.68 \mathrm{~mL}, 7.13 \mathrm{mmol})$. The reaction was stirred at room temperature for 5 hours and subsequently quenched with $\mathrm{H}_{2} \mathrm{O}(50 \mathrm{~mL})$. The product was extracted with EtOAc $(3 \times 75 \mathrm{~mL})$, dried over $\mathrm{Na}_{2} \mathrm{SO}_{4}$ and concentrated in vacuo. Yield: 1.35 $\mathrm{g}(88 \%)$ as a light yellow solid. The product was sufficiently pure according to ${ }^{1} \mathrm{H}-\mathrm{NMR}$ and was used without further purification. ${ }^{1} \mathrm{H}$ NMR (400 MHz, DMSO-d 6 ) $\delta \mathrm{ppm} 7.76(\mathrm{~d}, J=8.7 \mathrm{~Hz}, 1 \mathrm{H}), 7.02(\mathrm{~m}$, $2 \mathrm{H}$ ), 6.49 (s, 1H), 4.99 (s, 2H), 3.87 (s, 3H). ${ }^{13} \mathrm{H}$ NMR (75 MHz, $\mathrm{CDCl}_{3}$ ) $\delta$ ppm 163.0, 160.7, 155.8, 149.6, 125.1, 112.7, 112.6, 110.8, 101.2, 55.8, 41.3. HRMS calcd for $\mathrm{C}_{11} \mathrm{H}_{9} \mathrm{ClO}_{3}[\mathrm{M}]^{+\bullet} 224.0240$, found 224.0236 .

\section{4-Azidomethyl-7-methoxycoumarine}

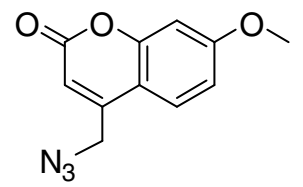

To a suspension of $\mathrm{NaN}_{3}(1.95 \mathrm{~g}, 30 \mathrm{mmol})$ in acetone $(100 \mathrm{~mL})$ and acetonitril $(100 \mathrm{~mL})$, 4chloromethyl-7-methoxycoumarine $(1.35 \mathrm{~g}, 6 \mathrm{mmol})$ was added. The mixture was stirred for 16 hours at room temperature after which a fraction of the crude reaction mixture was checked by ${ }^{1} \mathrm{H}$ NMR, 
displaying a conversion of roughly $60 \%$. An additional $1.95 \mathrm{~g}(30 \mathrm{mmol})$ of $\mathrm{NaN}_{3}$ was added to the reaction mixture which was stirred for an additional 48 hours. The volume of the reaction was reduced (approximately 50\%) by evaporation of the solvents in vacuo. The crude mixture was then suspended in EtOAc $(50 \mathrm{~mL})$ and the precipitated salts were removed by filtration. The resulting organic layer was dried over $\mathrm{Na}_{2} \mathrm{SO}_{4}$ and solvents were removed under reduced pressure to afford a light brown powder. Crude yield: $1.23 \mathrm{~g}$ (95\%). Gradient column chromatography (100\% Heptane $\rightarrow$ 65:35 EtOAc/Heptane) provided the pure title compound $(454 \mathrm{mg}, 34 \%)$ as a light yellow solid. ${ }^{1} \mathrm{H}$ NMR $\left(400 \mathrm{MHz}, \mathrm{CD}_{3} \mathrm{OD}\right) . . \delta$ ppm $7.62(\mathrm{~d}, J=8.7 \mathrm{~Hz}, 1 \mathrm{H}), 6.94(\mathrm{~m}, 2 \mathrm{H}), 6.34$ (s, 1H), 4.69 (s, 2H), 3.88 (s, 3H). ${ }^{13} \mathrm{H}$ NMR (75 MHz, $\left.\mathrm{CDCl}_{3}\right) \delta$ ppm 162.9, 160.6, 155.6, 148.6, 124.7, 112.7, 111.4, 110.7, 101.1, 55.8, 50.7. HRMS calcd for $\mathrm{C}_{11} \mathrm{H}_{9} \mathrm{~N}_{3} \mathrm{O}_{3}[\mathrm{M}]^{+\bullet}$ 231.0644, found 231.0635

\section{7-(1-Propyn)oxycoumarin}

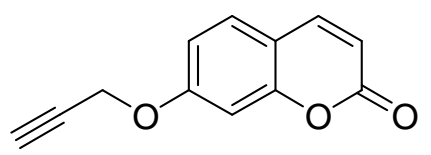

According to literature procedure. ${ }^{6}$ Quantities used: 7-hydroxycoumarin $(810 \mathrm{mg}, 5 \mathrm{mmol})$, propargyl

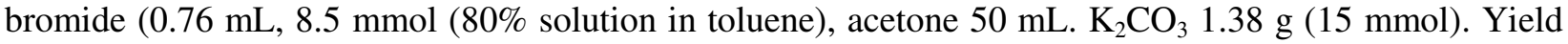
$870 \mathrm{mg}(87 \%)$ as a white solid. The analytical data are in agreement with literature values. ${ }^{1} \mathrm{H}$ NMR (400 MHz, DMSO-d $\left.{ }_{6}\right) \delta$ ppm $8.00(\mathrm{~d}, J=9.5 \mathrm{~Hz}, 1 \mathrm{H}), 7.66(\mathrm{~d}, J=8.6 \mathrm{~Hz}, 1 \mathrm{H}), 7.05(\mathrm{~d}, J=2.4 \mathrm{~Hz}, 1 \mathrm{H}), 6.99$ $(\mathrm{ddd}, J=8.6,2.5,0.7 \mathrm{~Hz}, 1 \mathrm{H}), 6.32(\mathrm{~d}, J=9.5 \mathrm{~Hz}, 1 \mathrm{H}), 4.93(\mathrm{~d}, J=2.4 \mathrm{~Hz}, 2 \mathrm{H}), 3.65(\mathrm{~m}, 1 \mathrm{H}){ }^{13} \mathrm{H} \mathrm{NMR}$ $\left(75 \mathrm{MHz}, \mathrm{CDCl}_{3}\right) \delta \mathrm{ppm} 160.9,160.4,155.5,143.2,128.8,113.5,112.9,102.0,77.3,76.5,56.1$. HRMS calcd for $\mathrm{C}_{12} \mathrm{H}_{9} \mathrm{O}_{3}[\mathrm{M}+\mathrm{H}]^{+}$201.0552, found 201.0546.

\section{5'-O-Tosyl-2'- $O$-methyl-6- $\mathrm{N}$-benzoyladenosine}

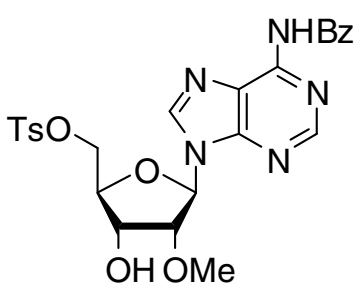

2'-O-methyl-6- $N$-benzoyladenosine ${ }^{11}(250 \mathrm{mg}, 0.65 \mathrm{mmol})$ was coevaporated 3 times with anhydrous pyridine and dissolved in pyridine $(4 \mathrm{~mL})$. To this solution $p \mathrm{TsCl}(120 \mathrm{mg}, 0.63 \mathrm{mmol})$ was added. The mixture was allowed to stir for 20 hours at $0{ }^{\circ} \mathrm{C}$. The reaction mixture was diluted with $\mathrm{CH}_{2} \mathrm{Cl}_{2}(20 \mathrm{~mL})$ and washed twice with a $5 \%$ aqueous $\mathrm{NaHCO}_{3}$ solution $(10 \mathrm{~mL})$. The organic layer was dried over $\mathrm{Na}_{2} \mathrm{SO}_{4}$. After concentration in vacuo and purification by column chromatography (acetone $/ \mathrm{CH}_{2} \mathrm{Cl}_{2}, 5: 95$ followed by acetone/ $\mathrm{CH}_{2} \mathrm{Cl}_{2} / \mathrm{MeOH}, 5: 93: 2$ and acetone/ $\left.\mathrm{CH}_{2} \mathrm{Cl}_{2} / \mathrm{MeOH}, 5: 91.5: 3.5\right)$ the title compound 
(160 mg, 30\%) was obtained as a white solid. ${ }^{1} \mathrm{H}$ NMR (300 MHz, DMSO) $\delta 11.22$ (s, 1H), 8.67 (s, 1H), 8.55 (s, 1H), 8.03 (d, J= 7.5 Hz, 1H). 7.69-7.54 (m, 5H), 7.32 (d, J= 8.1 Hz), 6.09 (d, J=4.8 Hz, 1H), 5.53 $(\mathrm{d}, J=5.4 \mathrm{~Hz}, 1 \mathrm{H}), 4.44-4.08(\mathrm{~m}, 5 \mathrm{H}), 3.03$ (s, 3H), 2.32 (s, 3H). ${ }^{13} \mathrm{C}$ NMR $(75 \mathrm{MHz}, \mathrm{DMSO}) \delta 165.6$, $151.8,151.7,150.5,145.0,143.13,133.3,132.5,131.9,129.9,128.5,127.5,125.8,86.0,81.9,81.4,69.9$, $68.6,57.3,21.0$.

\section{5'-Azido-5'-deoxy-2'-O-methyl-6- $N$-benzoyladenosine}

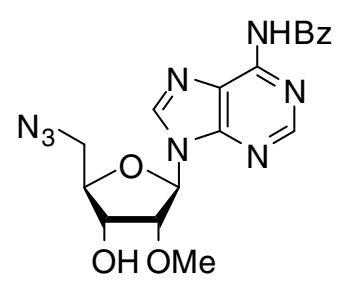

5'-O-Tosyl-2'-O-methyl-6- $N$-benzoyladenosine $(1.0 \mathrm{~g}, 1.85 \mathrm{mmol})$ was dissolved in $20 \mathrm{~mL} \mathrm{DMF}$ and $\mathrm{NaN}_{3}(750 \mathrm{mg}, 11.53 \mathrm{mmol})$ was added. The mixture was subsequently stirred at $88^{\circ} \mathrm{C}$ for $2 \mathrm{~h}$. After cooling to room temperature, the reaction mixture was diluted with $50 \mathrm{~mL}$ of $\mathrm{CH}_{2} \mathrm{Cl}_{2}$. The organic layer was washed with water $\left(3 \times 15 \mathrm{~mL}\right.$ ), dried with $\mathrm{Na}_{2} \mathrm{SO}_{4}$ and concentrated in vacuo. Crystallization from ethanol gave the title compound ( $690 \mathrm{mg}, 91 \%$ ) as a with solid. ${ }^{1} \mathrm{H}$ NMR (300 MHz, DMSO) $\delta 11.20$ (s, $1 \mathrm{H}), 8.76(\mathrm{~s}, 1 \mathrm{H}), 8.71(\mathrm{~s}, 1 \mathrm{H}), 8.03(\mathrm{~d}, J=7.2 \mathrm{~Hz}, 1 \mathrm{H}), 6.17$ (d, J=5.7 Hz, 1H), 5.50 (d, J=5.7 Hz, 1H), $4.58(\mathrm{t}, J=5.3 \mathrm{~Hz}, 1 \mathrm{H}), 4.56-4.41(\mathrm{~m}, 1 \mathrm{H}), 4.11-4.08(\mathrm{~m}, 1 \mathrm{H}), 3.69-3.62(\mathrm{~m}, 2 \mathrm{H}), 3.61(\mathrm{~s}, 3 \mathrm{H}) .{ }^{13} \mathrm{C} \mathrm{NMR}$ (75 MHz, DMSO) $\delta 165.6,152.0,151.8,150.5,143.3,133.3,132.3,128.5,125.8,85.9,83.6,81.5,69.4$, 57.7, 51.5. HRMS calcd for $\mathrm{C}_{18} \mathrm{H}_{18} \mathrm{~N}_{8} \mathrm{O}_{4}\left(\mathrm{M}+\mathrm{Na}^{+}\right) 433.1349$ found 433.1329 .

\section{2',3'-O,O-Isopropylidene-6- $N$-benzoyladenosine}

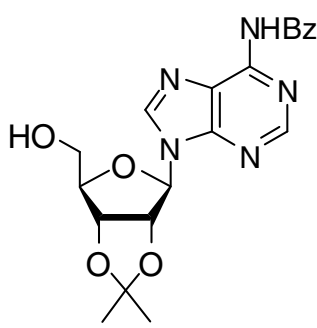

Commercial 2',3'-isopropylidene protected adenosine (307 $\mathrm{mg}, 1.0 \mathrm{mmol}$ ) was dried by coevaporation with pyridine and subsequently dissolved in pyridine $(5 \mathrm{~mL})$ and treated with TMSCl ( $0.64 \mathrm{~mL}, 5 \mathrm{mmol})$. The mixture was stirred at room temperature for 30 minutes followed by the addition of benzoyl chloride $(150 \mu \mathrm{L}, 1.3 \mathrm{mmol})$. After stirring at room temperature for 3 hours, the mixture was cooled to $0{ }^{\circ} \mathrm{C}$ and diluted with water $(1 \mathrm{~mL})$ and after 10 minutes a $\mathrm{NH}_{3}$-solution $(2 \mathrm{~mL})$ was added. The mixture was allowed to stir for 30 minutes at ambient temperature and evaporated to dryness. The residue was further purified by column chromatography $\left(\mathrm{CHCl}_{3} / \mathrm{MeOH}, 95: 5\right)$ and furnished $\mathbf{6}$ ( $380 \mathrm{mg}, 92 \%$ ) as a colorless foam. ${ }^{1} \mathrm{H}$ NMR (300 MHz, DMSO) $\delta 11.20$ (s, 1H), 8.74, (s, 1H), 8.65 (s, 1H), 7.93 (d, J= 8.1 Hz, 2H), 
7.86-7.44 (m, $3 \mathrm{H}), 6.25(\mathrm{~s}, 1 \mathrm{H}), 5.43-5.41(\mathrm{~m}, 1 \mathrm{H}), 5.12-5.11(\mathrm{~m}, 1 \mathrm{H}), 5.09-4.96(\mathrm{~m}, 1 \mathrm{H}), 4.25(\mathrm{bs}, 1 \mathrm{H})$, 3.54 (bs, $2 \mathrm{H}), 1.55$ (s, 3H), 1.33 (s, 3H). ${ }^{13} \mathrm{C}$ NMR (75 MHz, DMSO) $\delta 165.6,151.7,150.4,143.0,133.3$, $132.4,128.4,89.6,84.8,89.6,84.8,83.4,81.5,79.7,69.2,57.8,54.9,27.0,25.1$. HRMS calcd for $\mathrm{C}_{20} \mathrm{H}_{21} \mathrm{~N}_{5} \mathrm{O}_{5}\left(\mathrm{M}+\mathrm{Na}^{+}\right) 434.14404$ found 434.1432 .

\section{5'-O-Propargyl-2',3'-O,O-isopropylidene-6- $N$-benzoyladenosine}

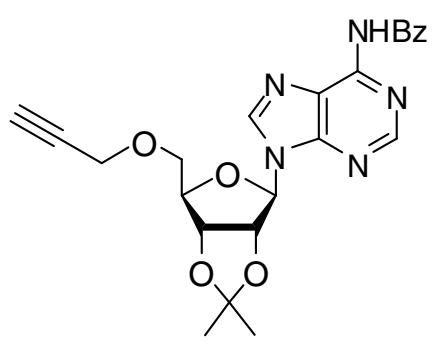

2',3'-O,O-Isopropylidene-6- $N$-benzoyladenosine (6) $(400 \mathrm{mg}, 0973 \mathrm{mmol}$ ) was dissolved in dry THF $(10 \mathrm{~mL})$, cooled to $0{ }^{\circ} \mathrm{C}$ before addition of $\mathrm{NaH}(70 \mathrm{mg}, 1.75 \mathrm{mmol}, 60 \%$ dispersion in mineral oil). This mixture was subsequently stirred for 40 minutes after which TBAI (120 mg, $0.325 \mathrm{mmol})$ and propargyl bromide $(150 \mu \mathrm{L}, 1.75 \mathrm{mmol})$. After $20 \mathrm{~h}$ of stirring at room temperature, the reaction mixture was evaporated to dryness and adsorbed on silica. Purification by column chromatography $\left(\mathrm{CH}_{2} \mathrm{Cl}_{2} / \mathrm{MeOH}\right.$, 98:2) yielded the title compound 7 (196.8 $\mathrm{mg}, 45 \%$ ) as a white solid. ${ }^{1} \mathrm{H}$ NMR (300 MHz, DMSO) $\delta 11.18$ (s, 1H), $8.75(\mathrm{~s}, 1 \mathrm{H}), 8.60(\mathrm{~s}, 1 \mathrm{H}), 8.03$ (d, J=7.2 Hz, 2H), 7.66-7.50 (m, 3H), 6.29 (d, J=2.1 Hz, 1H), 5.48-5.45 (m, 1H), 5.02-4.99 (m, 2H) 4.39-4.35 (m, 1H), $4.11(\mathrm{~d}, J=2.4 \mathrm{~Hz}), 3.67-3.55(\mathrm{~m}, 2 \mathrm{H}), 3.40(\mathrm{~s}$, $1 \mathrm{H}), 1.54 \mathrm{c}(\mathrm{s}, 2 \mathrm{H}), 1.33$ (s, 1H). ${ }^{13} \mathrm{C}$ NMR (75 MHz, DMSO) $\delta 165.6,151.7,150.4,143.0,133.3,132.3$, 128.4, 89.6, 84.8, 83.4, 81.5, 79.7, 69.2, 57.8, 54.9, 27.0, 25.1. HRMS calcd for $\mathrm{C}_{23} \mathrm{H}_{23} \mathrm{~N}_{5} \mathrm{O}_{5}\left(\mathrm{M}+\mathrm{Na}^{+}\right)$ 472.1597 found 472.1578 . 
General procedure for the copper(I)-catalyzed azido-alkyne cycloaddition (CuAAC).

The acetylene (2a) or azido (2b)-functionalized adenosine $(0.2 \mathrm{mmole})$ and 1 equivalent of an azido or acetylene reaction partner, respectively, were dissolved in a mixture of acetonitrile $(2 \mathrm{~mL})$ and water $(0.2$ $\mathrm{mL}$ ). The reaction mixture stirred at $35^{\circ} \mathrm{C}$ in the presence of a Cu-wired stirring bar. After $1 \mathrm{~h}$, the stirring bar was removed and stirring was prolonged until all starting material was consumed. Concentration in vacuo and purification by flash chromatography $\left(\mathrm{CH}_{2} \mathrm{Cl}_{2} / \mathrm{MeOH}, 95: 5\right)$ furnished the triazolefunctionalized nucleosides. 
Entry 1. CuAAC-adduct of 2a and benzyl azide

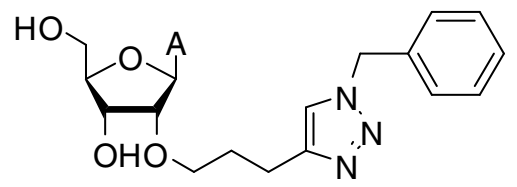

${ }^{1} \mathrm{H}$ NMR (300 MHz, DMSO) $\delta 8.37$ (s, 1H), 8.11 (s, 1H), 7.74 (s, 1H), 7.34-7.25 (m, 7H), 5.99 (d, $J=6.3$ $\mathrm{Hz}, 1 \mathrm{H}), 5.49$ (s, 2H), 5.39 (m, 1H), 5.29 (d, J = 4.8 Hz, 1H), 4.45 (m, 1H), 4.30 (m, 4H), 3.98 (m, 1H), 3.68-3.53 (m, 2H), 3.38-3.30 (m, 2H), 2.55 (m, 2H), 1.76 (m, 2H). ${ }^{13} \mathrm{C}$ NMR (75 MHz, DMSO) . 156.1, 152.5, 149.0, 146.6, 139.7, 136.2, 128.7, 128.0, 127.8, 122.0, 119.3, 86.3, 86.0, 80.9, 68.9, 68.7, 61.4, 52.6, 28.9, 21.3. HRMS calcd for $\mathrm{C}_{22} \mathrm{H}_{27} \mathrm{~N}_{8} \mathrm{O}_{4}\left(\mathrm{M}+\mathrm{H}^{+}\right)$467.2153 found 467.2166.

Entry 2. CuAAC-adduct of 2 a and 2,3,4,6-tetra- $O$-acetyl- $\beta$-D-glucopyranosyl azide

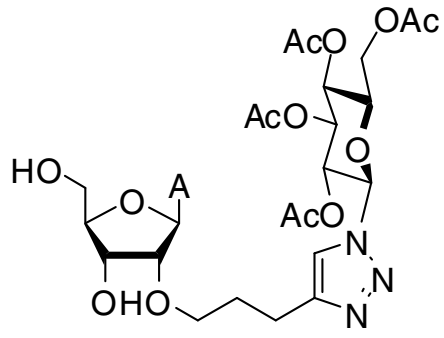

${ }^{1} \mathrm{H}$ NMR (300 MHz, DMSO) $\delta 8.36$ (s, 1H), 8.11 (s, 1H), 8.05 (s, 1H), 7.3 (s, 2H), 6.23 (d, J= $8.7 \mathrm{~Hz}$ ), $5.99(\mathrm{~d}, J=6.3 \mathrm{~Hz}), 5.40(\mathrm{~m}, 2 \mathrm{H}), 5.30(\mathrm{~m}, 3 \mathrm{H}), 5.11$ (d, $J=5.4 \mathrm{~Hz}), 4.60(\mathrm{~m}, 1 \mathrm{H}), 4.77-4.56(\mathrm{~m}, 1 \mathrm{H}), 4.31-$ 4.304 .06 (dd, $J=36.0 \mathrm{~Hz}, J=14.8 \mathrm{~Hz}, 1 \mathrm{H}), 3.98$ (d, $J=3 \mathrm{~Hz}, 1 \mathrm{H}), 5.43-5.10$ (m, 6H), 3.69-3.61 (m, 5H), 3.46-3.36 (m, 3H), 2.58-2.48 (m, 2H), $1.74(\mathrm{~m}, 2 \mathrm{H}) .{ }^{13} \mathrm{C}$ NMR (75 MHz, DMSO) $\delta 170.0,169.5,169.3$, 168.4, 156.1, 152.5, 149.0, 147.0,120.9, 86.3, 86.1, 83.7, 80.9, 73.2, 72.1, 70.1, 69.0, 68.7, 67.6, 61.8, 61.4, 28.8, 21.3, 20.5, 20.4, 20.2, 19.8. HRMS calcd for $\mathrm{C}_{29} \mathrm{H}_{38} \mathrm{~N}_{8} \mathrm{O}_{13}\left(\mathrm{M}+\mathrm{Na}^{+}\right)$729.2451 found 729.2480.

\section{Entry 3. CuAAC-adduct of 2a and 4-azidomethyl-7-methoxycoumarine}

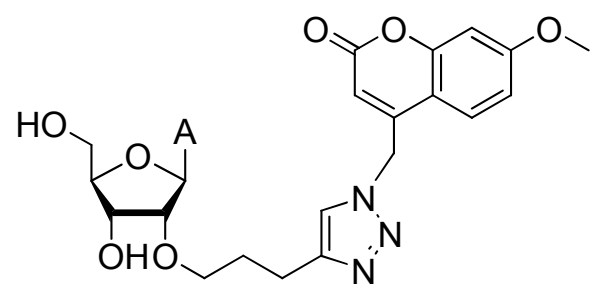


${ }^{1} \mathrm{H}$ NMR (300 MHz, DMSO) $\delta 8.36$ (s, 1H), 8.10 (s, 1H), 7.88 (s, 1H), 7.72 (d, J= 8.7 Hz), 7.32 (s, 2H), 7.02-6.95 (m, 2H), 5.98 (d, J=6.0 Hz), 5.83 (s, 2H), 5.61 (s. 1H), 5.39 (bs, 1H), 5.26(bs, 1H), 4.45 (m, $1 \mathrm{H}), 4.29(\mathrm{~m}, 1 \mathrm{H}), 3.97(\mathrm{~m}, 1 \mathrm{H}), 3.84(\mathrm{~s}, 3 \mathrm{H}), 3.68-3.56(\mathrm{~m}, 3 \mathrm{H}), 3.48-3.41(\mathrm{~m}, 1 \mathrm{H}), 2.59$ (d, J=2.4 Hz), 1.73 (t, J=4.9 Hz. ${ }^{13} \mathrm{C}$ NMR (75 MHz DMSO) $\delta 162.7,159.8,156.1,155.0,152.5,149.0,146.9,139.7$, 125.8, 123.0, 119.3, 112.4, 110.5, 110.3, 110.1, 56.3, 86.0, 80.9, 68.9, 68.7, 61.4, 55.9, 49.0, 28.9, 21.3 . HRMS calcd for $\mathrm{C}_{22} \mathrm{H}_{28} \mathrm{~N}_{8} \mathrm{O}_{7}\left(\mathrm{M}+\mathrm{Na}^{+}\right) 587.1973$ found 587.1948.

\section{Entry 4. CuAAC-adduct of 2a and 5'-azido-5'-deoxy-2'- $O$-methyl-6- $N$-benzoyladenosine}

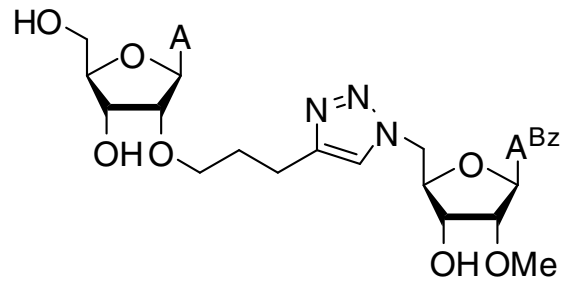

${ }^{1} \mathrm{H}$ NMR (300 MHz DMSO) $\delta 8.72(\mathrm{~s}, 1 \mathrm{H}), 8.59$ (s, 1H) 8.35 (s, 1H), 8.09 (s,1H), 8.01 (d,J=5.6 Hz, 2H), 7.64-7.59 (m, 2H), 7.54-7.50 (m, 2H), 7.31 (s, 2H),6.13 (d, J=3.5 Hz), 5.96 (d, J=4.5 Hz), 5.72-5.29 (bm, $3 \mathrm{H}, 4.70-4.66(\mathrm{~m}, 2 \mathrm{H}), 4.45-4.42(\mathrm{bm}, 2 \mathrm{H}), 4.27$ (as, 1H), $3.64(\mathrm{~m}, 2 \mathrm{H})) 3.61-3.51(\mathrm{~m}, 2 \mathrm{H}), 3.34(\mathrm{~s}, 3 \mathrm{H}) 1.06$ (m, 2H). ${ }^{13} \mathrm{C}$ NMR ( $75 \mathrm{MHz}$ DMSO) $\delta 165.8,156.1,152.5,151.8,150.6,149.0 .146 .3,143.2,140.2,139.7$, $133.4,132.4,128.5,128.4,125.8,122.6,122.6,119.3,86.3,86.0,82.9,81.5,80.9,69.5,68.3,68.7,61.5$, 57.4, 51.0, 30.7, 28.8, 21.2. HRMS calcd for $\mathrm{C}_{33} \mathrm{H}_{37} \mathrm{~N}_{13} \mathrm{O}_{8}\left(\mathrm{M}+\mathrm{Na}^{+}\right), 766.2786$ found 766.2781 .

\section{Entry 5 CuAAC-adduct of 2a and 5'-azido-5'-deoxyadenosine}

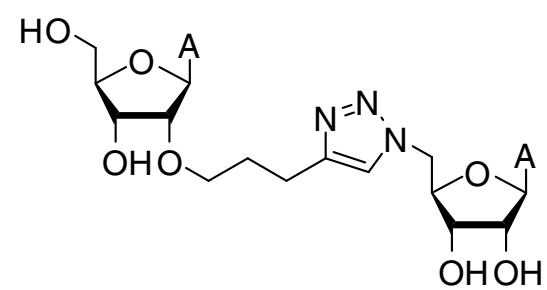

${ }^{1} \mathrm{H}$ NMR (300 MHz DMSO) $\delta$ 8.37, 8.17, 8.13.(s,1H), $8.11(\mathrm{~s}, 1 \mathrm{H}), 7.60$ (s, 1H), 7.32 (s, 2H) 5.98 (d, $J=$ $6.0 \mathrm{~Hz}, 2 \mathrm{H}), 5.97(\mathrm{~d} J=5.1 \mathrm{~Hz}), 5.79-5.29(\mathrm{bs}, 4 \mathrm{H}) 4.66-4.65(\mathrm{~m}, 3 \mathrm{H}) 4.46(\mathrm{t}, J=7.2,1 \mathrm{H}), 4.31(\mathrm{bs}, 1 \mathrm{H})$, 4.21 (bs, 2H), 3.97 (d, $J=3 \mathrm{~Hz}) 3.67-3.64(\mathrm{~m}, 3 \mathrm{H}), 1.72-1.61(\mathrm{~m}, 2 \mathrm{H}) .{ }^{13} \mathrm{C}$ NMR ( $\left.75 \mathrm{MHz} \mathrm{DMSO}\right) \delta$ 156.1, 156.0, 152.7, 152.5, 149.2, 149.0, 146.2, 139.8, 122.6, 119.3, 119.2, 87.7, 86.3, 86.0, 82.3, 80.8, 72.6, 70.9, 68.9, 68.7, 61.5, 60.6, 51.2, 28.9, 21,23. HRMS calcd for $\mathrm{C}_{25} \mathrm{H}_{31} \mathrm{~N}_{13} \mathrm{O}_{7}\left(\mathrm{M}+\mathrm{Na}^{+}\right), 648.2367$ found 648.2326 . 
Entry 6 CuAAC-adduct of 2a and 2'-azido-2'-deoxyuridine

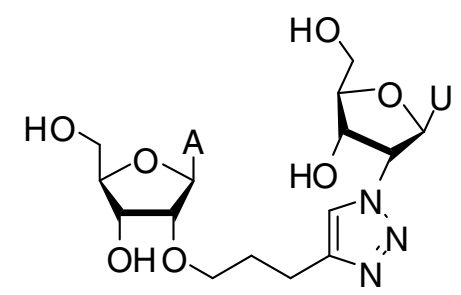

${ }^{1} \mathrm{H}$ NMR (300 MHz DMSO) $\delta 8.37$ (s, 1H), 8.12 (s, 1H), 7.89 (d, J=8.41Hz, 1H), 7.80 (s, 1H), 7.31 (s, 2H), 6.42 (d, J=6.3 Hz), 6.0 (d, J=6.3 Hz), 5.83 (bs, 1H), 5.69 (d, J=8.1 Hz, 1H), 5.41 (bs, 1H), 5.30-5.28 (m, 2H), 4.49-4.39 (m, 1H), 4.32 (bs, 1H), 4.08 (bs, 1H), 4.07 (d, J=3.6 Hz, 1H), 3.99 (d, J=3Hz, 1H), 3.69-3.38 (m, 4H), $3.31(\mathrm{~m}, 1 \mathrm{H}), 2.58-2.54(\mathrm{~m}, 2 \mathrm{H}), 1.76-1.73(\mathrm{~m}, 1 \mathrm{H}) .{ }^{13} \mathrm{C}$ NMR $(75 \mathrm{MHz}$ DMSO) $\delta$ 162.9, 156.3, 152.5, 150.3, 149.0, 145.9, 140.1, 139.7, 122.9, 119.0, 102.4, 86.4, 86.0, 85.9, 85.6, 80.9. 69.4, 69.0, 68.3, 64.7, 60.5, 29.0, 21.3. HRMS calcd for $\mathrm{C}_{24} \mathrm{H}_{30} \mathrm{~N}_{10} \mathrm{O}_{9}\left(\mathrm{M}+\mathrm{Na}^{+}\right)$625.2094 found 625.2055.

\section{Entry 7 CuAAC-adduct of 2a and 4-azido-2,2,6,6-tetramethylpiperidine 1-oxyl (TEMPO)}

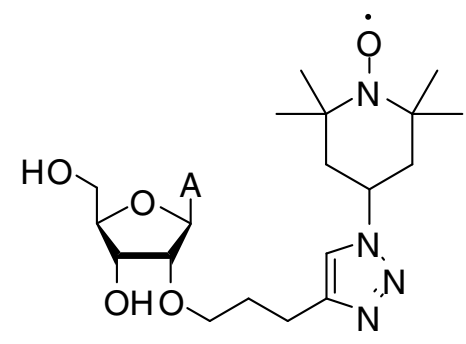

IR $\vee$ 3334, 3152, 2919, 1644, 1584, 1087. HRMS calcd for $\mathrm{C}_{24} \mathrm{H}_{36} \mathrm{~N}_{9} \mathrm{O}_{5}\left(\mathrm{M}+\mathrm{Na}^{+}\right) 553.2737$ found 553.2754. Mp $79.2{ }^{\circ} \mathrm{C}$.

Spectroscopic data of the CuAAC-adducts of $2 \mathrm{~b}$ with acetylene derivatives (Table 2, entries 1-5)

Entry 1. CuAAC-adduct of $2 \mathrm{~b}$ and propargyl alcohol

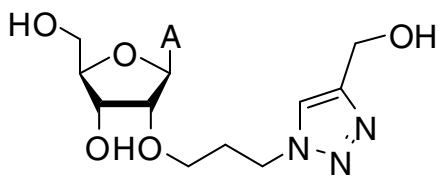


${ }^{1} \mathrm{H}$ NMR (300 MHz DMSO) $\delta 8.37$ (s, 1H), 8.12 (s, 1H), 7.85 (s, 1H), 7.32, (s ,2 H), 6.0 (d, J=6.0 Hz, 1H), 5.37 (bt, 1H), 5.24 (d, J=4.8 Hz), 4.47-4.43 (m, 3H), 4.34-4.30 (m, 3H), 3.98 (d, J=3 Hz, 1H), 3.63$3.53(\mathrm{~m}, 2 \mathrm{H}), 3.51-3.36(\mathrm{~m}, 2 \mathrm{H}), 1.96,(\mathrm{~m}, 2 \mathrm{H}) .{ }^{13} \mathrm{C}$ NMR ( $75 \mathrm{MHz}$ DMSO) $\delta 156.14,152.1,149.0$, 147.8, 139.6, 122.7, 119.3, 86.2, 86.0, 68.9, 66.5, 61.3, 55.0, 46.2, 30.0. HRMS calcd for $\mathrm{C}_{16} \mathrm{H}_{22} \mathrm{~N}_{8} \mathrm{O}_{5}(\mathrm{M}$ $+\mathrm{Na}^{+}$) 429.1611 found 429.1599 . 


\section{Entry 2. CuAAC-adduct of $2 \mathrm{~b}$ and 1,7-octadiyne}

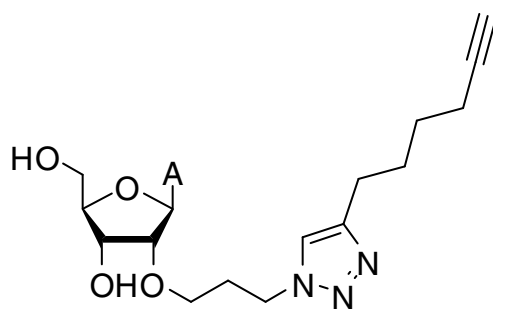

${ }^{1} \mathrm{H}$ NMR (300 MHz DMSO) $\delta 8.44(\mathrm{~s}, 1 \mathrm{H}), 8.23(\mathrm{~s}, 1 \mathrm{H}), 8.20(\mathrm{~s}, 1 \mathrm{H}), 8.05(\mathrm{~d}, J=7.0 \mathrm{~Hz}, 1 \mathrm{H}), 7.70(\mathrm{~d}, J=$ $6.45 \mathrm{~Hz}, 1 \mathrm{H}), 7.41(\mathrm{~s}, 2 \mathrm{H}), 7.21(\mathrm{~d}, J=1.45 \mathrm{~Hz}, 1 \mathrm{H}), 7.08$ (dd, J=6.45 Hz, 1.7 Hz, 1H), 6.36 (d, J= 7.0 Hz, $1 \mathrm{H}), 6.08(\mathrm{~d}, J=4.4 \mathrm{~Hz}), 5.46(\mathrm{bs}, 1 \mathrm{H}), 5.32(\mathrm{~d}, J=3.8 \mathrm{~Hz}, 1 \mathrm{H}), 4.52(\mathrm{~m}, 1 \mathrm{H}), 4.45(\mathrm{~m}, 1 \mathrm{H}), 4.39(\mathrm{~m}, 2 \mathrm{H})$, $4.06(\mathrm{~d}, J=2.5 \mathrm{~Hz}), 3.73-3.48(\mathrm{~m}, 2 \mathrm{H}), 3.47-3.39(\mathrm{~m}, 2 \mathrm{H}) 2.08(\mathrm{t}, J=5.0 \mathrm{~Hz}) .{ }^{13} \mathrm{C}$ NMR $(75 \mathrm{MHz} \mathrm{DMSO})$ $\delta 161.1,160.2,156.1,155.3,152.5149 .0,144.3,141.8,139.6,129.5,124.8,119.3,112.8,112.7,112.6$, 101.5, 86.1, 86.1, 811, 68.9, 66.5, 61.7, 61.3, 54.9, 46.5, 29.9. HRMS calcd for $\mathrm{C}_{25} \mathrm{H}_{26} \mathrm{~N}_{8} \mathrm{O}_{7}\left(\mathrm{M}+\mathrm{Na}^{+}\right)$ 573.1822 found 573.1793 .

Entry 3. CuAAC-adduct of 2b and 7-(1-propyn)oxycoumarin

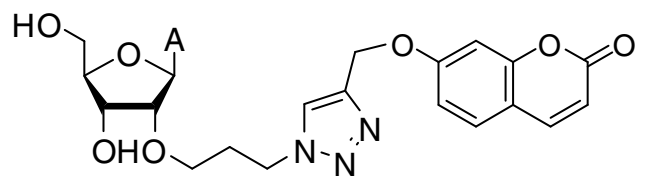

${ }^{1} \mathrm{H}$ NMR (300 MHz DMSO) $\delta 8.48(\mathrm{~s}, 1 \mathrm{H}), 8.23(\mathrm{~s}, 1 \mathrm{H}), 7.97$ (s, 2H), $7.6(\mathrm{~s}, 1 \mathrm{H}), 6.01(\mathrm{~d}, J=4.3 \mathrm{~Hz}, 1 \mathrm{H})$, $4.38(\mathrm{t}, J=7.2 \mathrm{~Hz}, 1 \mathrm{H}), 4.30-4.27$ (m, 3H), 3.98 (d, $J=2.6 \mathrm{~Hz}, 1 \mathrm{H}), 3.64-3.52(\mathrm{~m}, 3 \mathrm{H}), 3.35-3.32(\mathrm{~m}, 1 \mathrm{H})$, $2.69(\mathrm{~s}, 1 \mathrm{H}), 2.53(\mathrm{t}, J=5.7 \mathrm{~Hz}, 2 \mathrm{H}), 2.14(\mathrm{~m}, 2 \mathrm{H}), 1.94(\mathrm{~m}, 2 \mathrm{H}), 1.44(\mathrm{~m}, 2 \mathrm{H}), 1.19(\mathrm{~m}, 2 \mathrm{H}) .{ }^{13} \mathrm{C} \mathrm{NMR}($ $75 \mathrm{MHz}$ DMSO) $\delta$ 154.2, 150.1, 148.7, 146.5, 140.4, 121.8, 119.1, 86.2, 86.1, 84.4, 81.3, 71.2, 68.8, 66.4, 61.2, 46.08, 29.9, 28.0, 27.4, 24.4, 17.4. HRMS calcd for $\mathrm{C}_{21} \mathrm{H}_{28} \mathrm{~N}_{8} \mathrm{O}_{4}\left(\mathrm{M}+\mathrm{Na}^{+}\right)$479.2131 found 479.2115.

\section{Entry 4. CuAAC-adduct of $2 \mathrm{~b}$ and $5^{\prime}-O$-propargyl-2',3'-O,O-isopropylidene-6- $N$-benzoyladenosine}

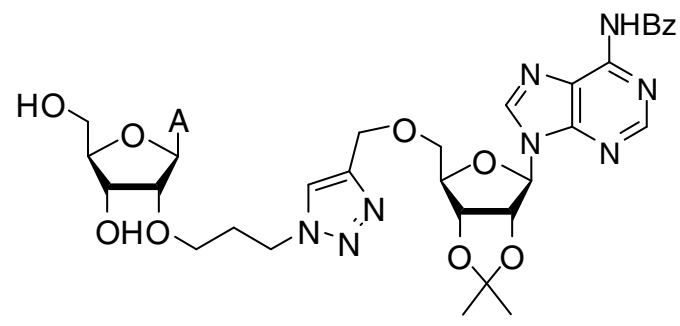


${ }^{1} \mathrm{H}$ NMR (300 MHz DMSO) $\delta 11.20$ (s, 1H), 8.72 (s, 1H), 8.55 (s, 1H), 8.35 (s, 1H), 8.10 (s, 1H), 8.03 (d, $J=7.2 \mathrm{~Hz}, 2 \mathrm{H}), 7.89$ (s, 1H), 7.61-751 (m, 3H), 7.32 (s, 2H), 6.25 (d, J=2.4 Hz, 1H), 5.98 (d, J= 5.7 Hz, 1H), $5.40(\mathrm{~m}, 2 \mathrm{H}), 5.21(\mathrm{~d}, J=5.1 \mathrm{~Hz}, 1 \mathrm{H}), 4.97(\mathrm{~d}, J=2.4 \mathrm{~Hz}, 1 \mathrm{H}), 4.45-4.30(\mathrm{~m}, 8 \mathrm{H}), 3.97$ (d, J=3.0 Hz, $1 \mathrm{H}), 3.62(\mathrm{~m}, 6 \mathrm{H}), 1.96(\mathrm{~m}, 2 \mathrm{H}), 1.52(\mathrm{~s}, 3 \mathrm{H}), 1.31(\mathrm{~s}, 3 \mathrm{H}) .{ }^{13} \mathrm{C}$ NMR ( $75 \mathrm{MHz}$ DMSO) $\delta$ 156.1, 152.5, $151.7,150.3,148.9,143.2,142.9,139.6,133.3,132.4,128.5,128.4,125.6,124.0,119.3,119.3,113.1$, $89.8,86.2,86.1,85.0,83.6,81.5,81.0,69.7,68.9,66.5,61.3,46.3,29.9,26.9,25.1$. HRMS calcd for $\mathrm{C}_{36} \mathrm{H}_{41} \mathrm{~N}_{13} \mathrm{O}_{9}\left(\mathrm{M}+\mathrm{H}^{+}\right) 800.3223$ found 800.3259 .

\section{Entry 5. CuAAC-adduct of $2 \mathrm{~b}$ and $(E)-4-[4-(($ dimethylamino)phenyl)-diazenyl]- $N$-(prop-2- ynyl)benzamide}

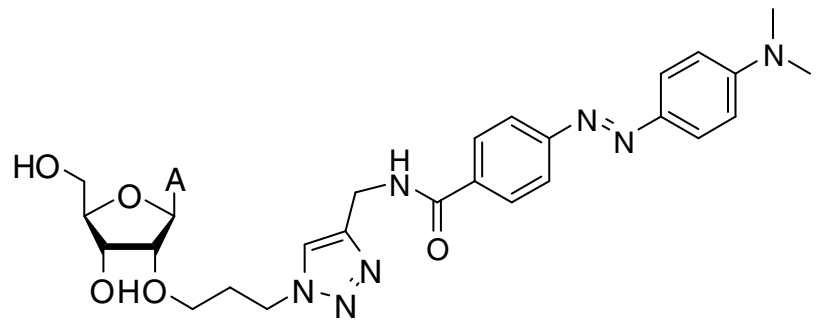

IR v 3299, 2954, 2915, 2850, 1631, 1601, 1515, 1359, 1134, 1082. ${ }^{1} \mathrm{H}$ NMR $(300 \mathrm{MHz} \mathrm{CDCl}) \delta 8.32(\mathrm{~s}$, 1H), 8.29 (s, 1H), 7.89-7.86 (m, 8H), 7.67 (s, 1H), 6.75 (d, J=8.6 Hz, 1H), 6.68-6.4 (m, 1H), 5.95-5.86 (m, 2H), 5.08-4.97 (m ,1H), 4.81-4.84 (m, 1H), $4.72(\mathrm{~m}, 2 \mathrm{H}), 4.56-4.52(\mathrm{~m}, 2 \mathrm{H}), 4.37(\mathrm{~m}, 2 \mathrm{H}), 3.95(\mathrm{~d}, J=13.0$ $\mathrm{Hz}, 2 \mathrm{H}), 3.77-3.69(\mathrm{~m}, 2 \mathrm{H}), 3.13(\mathrm{~m}, 2 \mathrm{H}) 3.10(\mathrm{~s}, 6 \mathrm{H}), 1.96(\mathrm{~m}, 2 \mathrm{H}) .{ }^{13} \mathrm{C}$ NMR ( $75 \mathrm{MHz}$ DMSO) $\delta 167$, 156.0, 155.3, 152.8, 152.5, 148.5, 143.6, 141.1, 140.9, 133.7, 132.9, 127.9, 125.4, 123.2, 122.3, 121.7, 119.0, 111.4, 89.7, 89.4, 88.5, 88.1, 81.2, 80.3, 72.2, 71.2, 70.3, 65.6, 63.3, 46.4, 40.3, 35.4, 30.2, 29.7.HRMS calcd for $\mathrm{C}_{31} \mathrm{H}_{36} \mathrm{~N}_{12} \mathrm{O}_{5}\left(\mathrm{M}+\mathrm{Na}^{+}\right) 679.2829$ found 679.2798 . 
HPLC-profile of oligonucleotide 5.
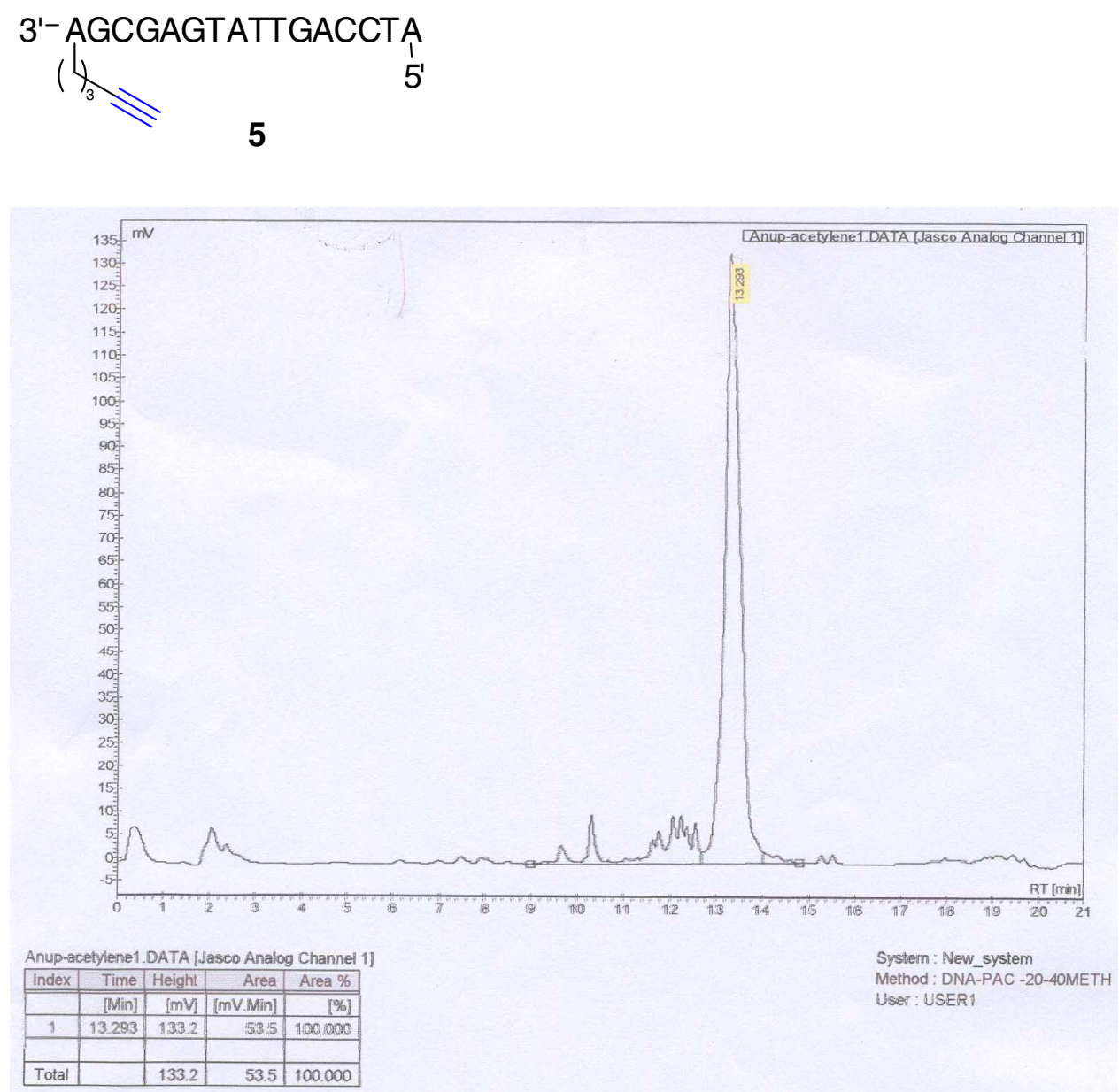

HPLC-profile of oligonucleotide 6.

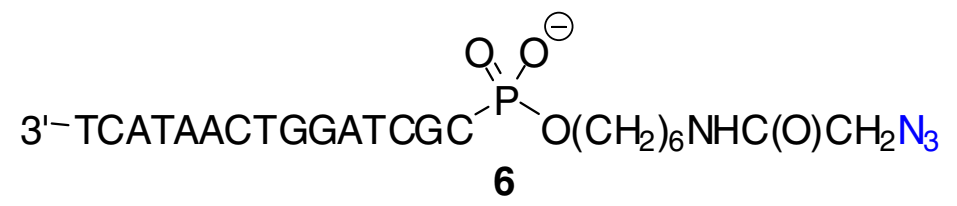




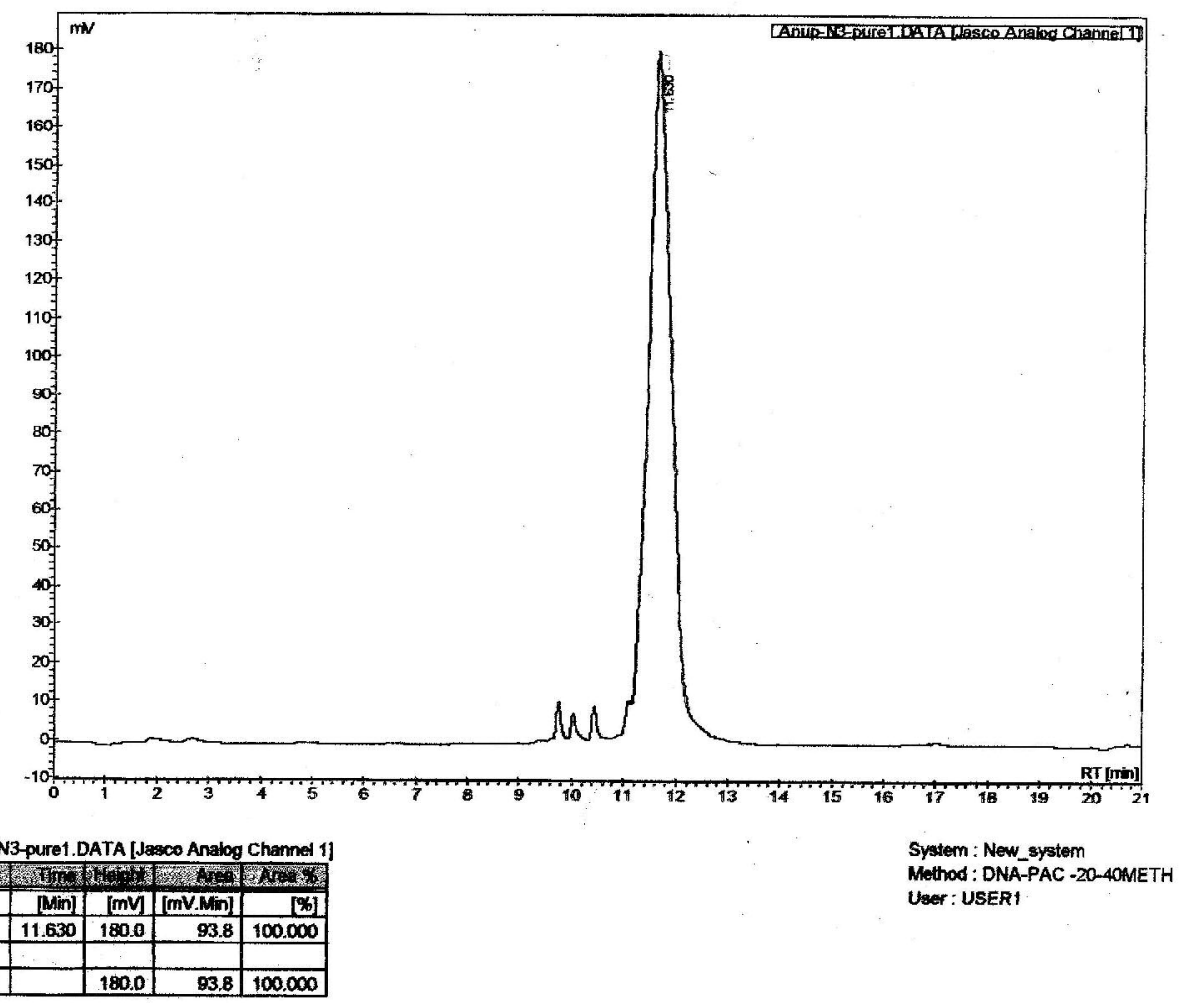

HPLC-profile of CuAAC reaction of 5 and 6, leading to triazole-linked ligonucleotide 7.

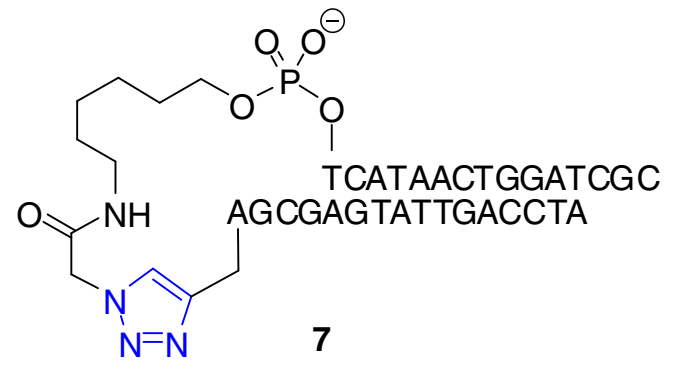

$\mathrm{T}=2$ hours: 


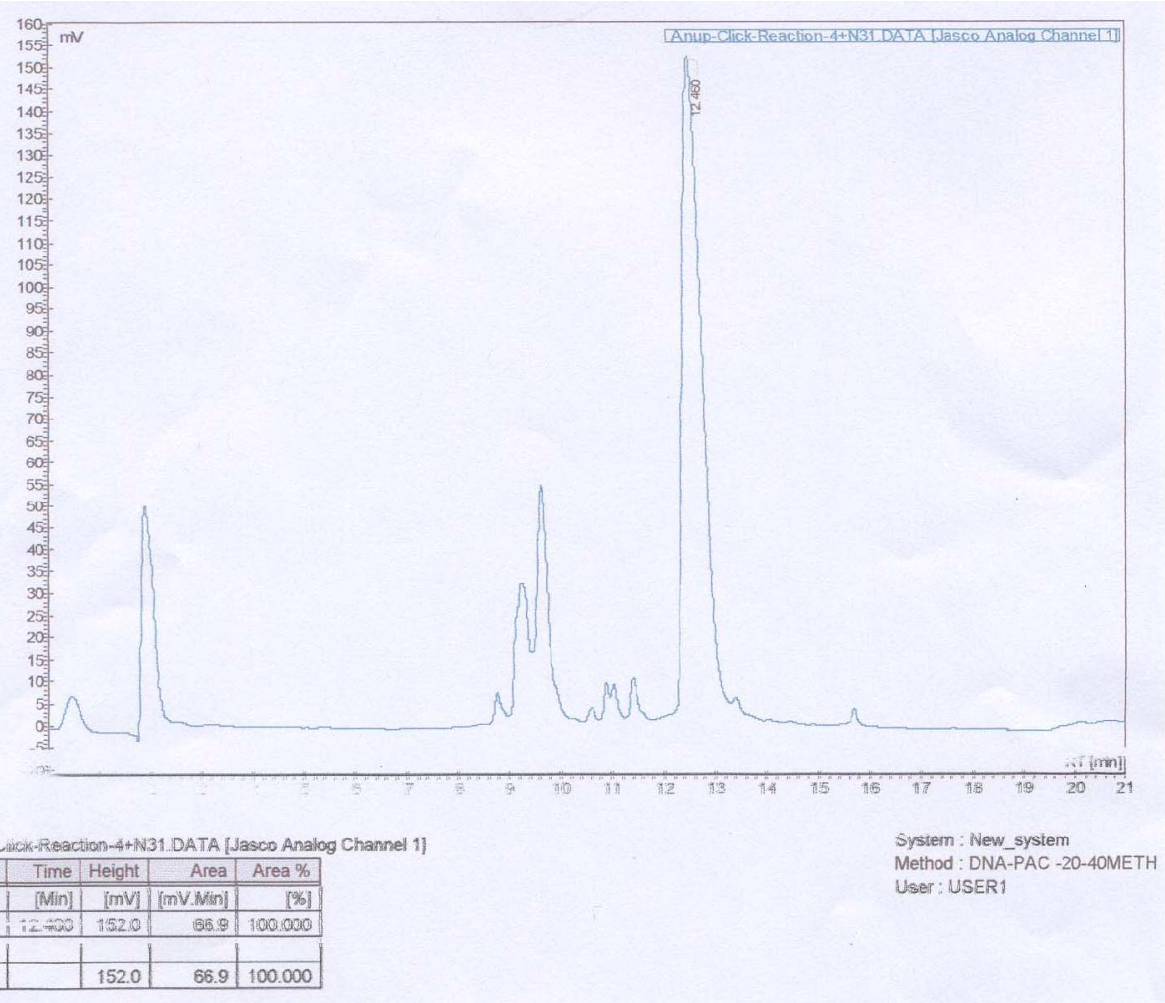

$\mathrm{T}=16$ hours

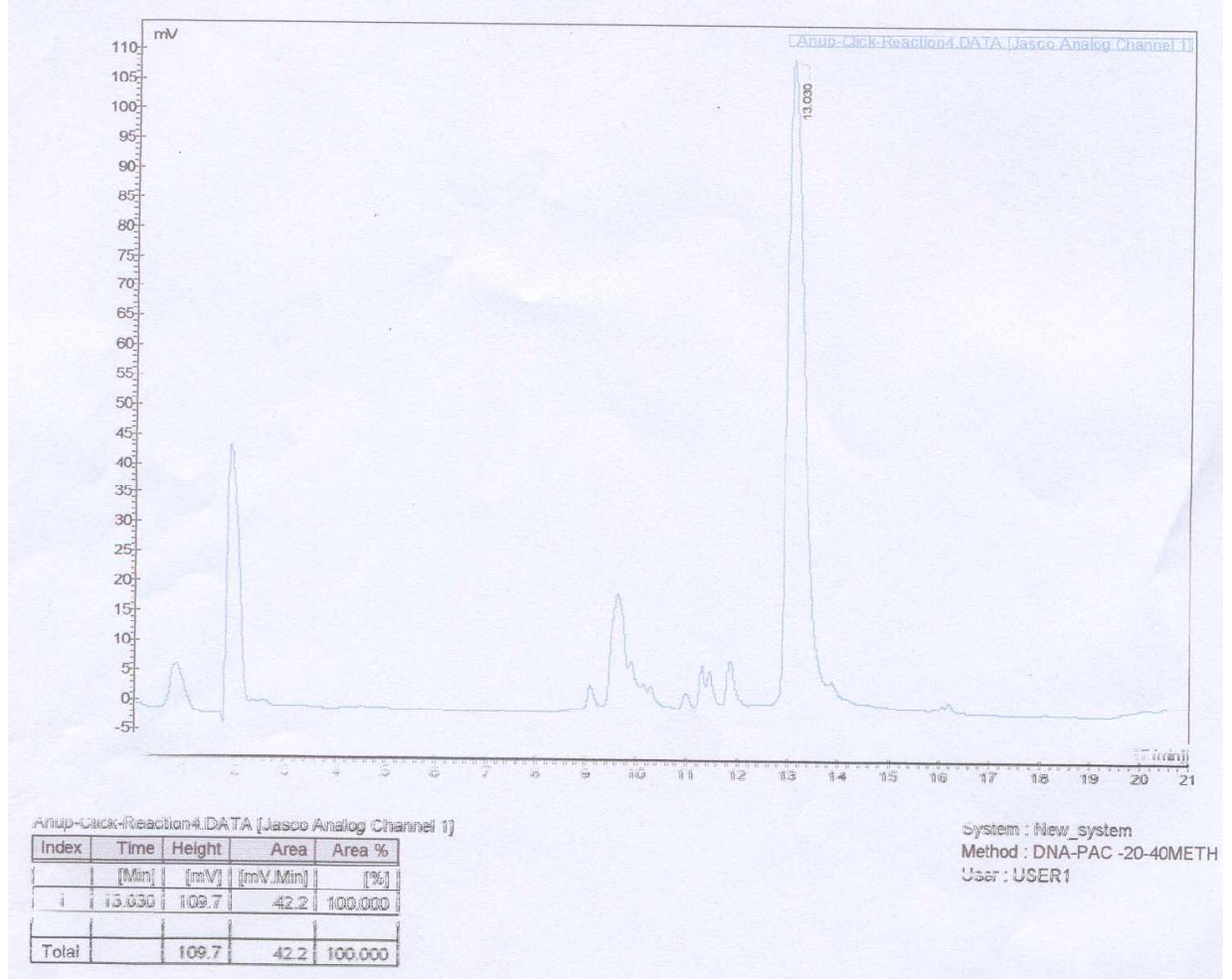

MALDI-TOF: calcd for $\mathrm{C}_{316} \mathrm{H}_{404} \mathrm{~N}_{121} \mathrm{O}_{186} \mathrm{P}_{30}$ : 9802.50 , found $9805.31(\mathrm{M}+\mathrm{H})$ 


\section{${ }^{1} \mathrm{H}$ and ${ }^{13} \mathrm{C}$ NMR spectra}

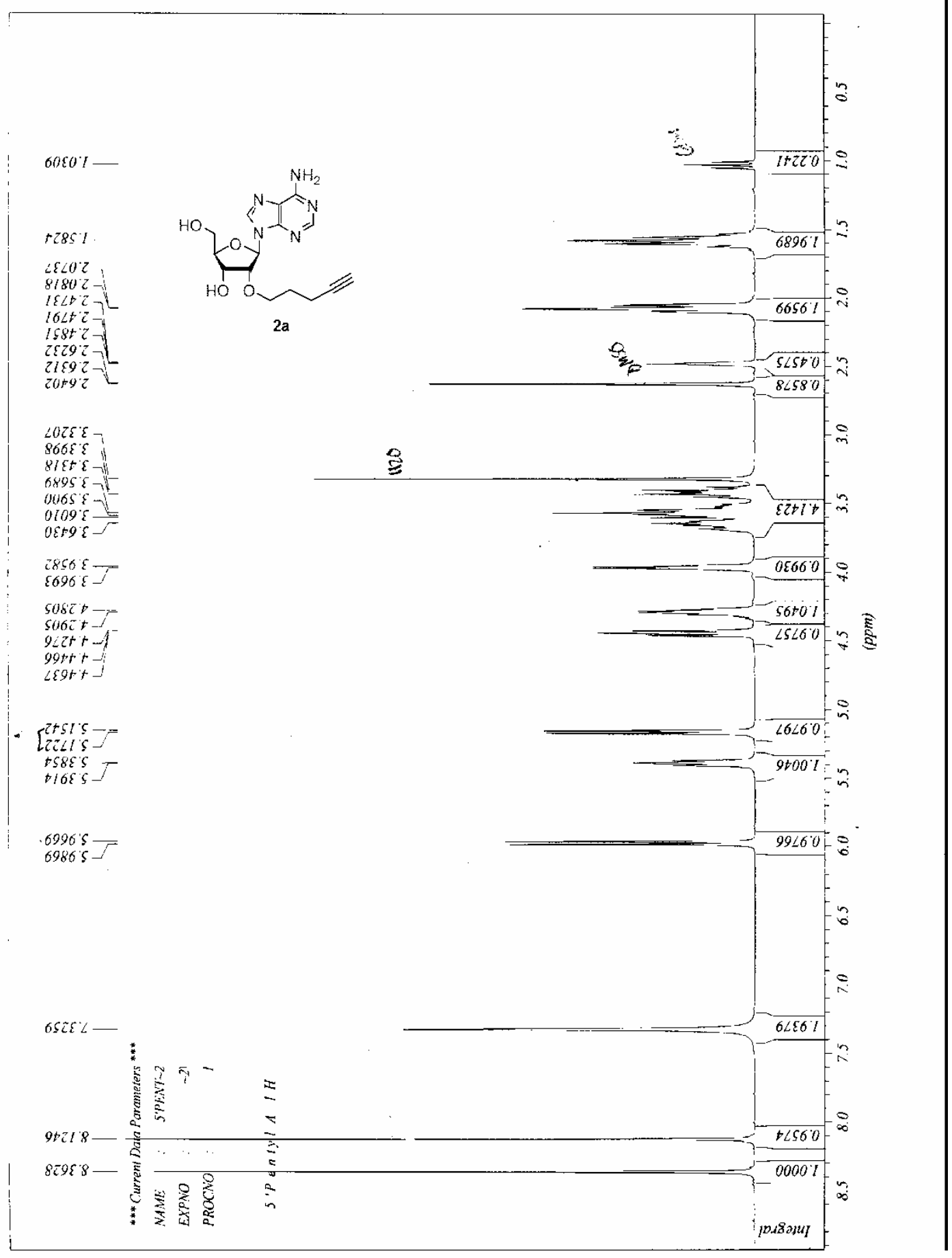




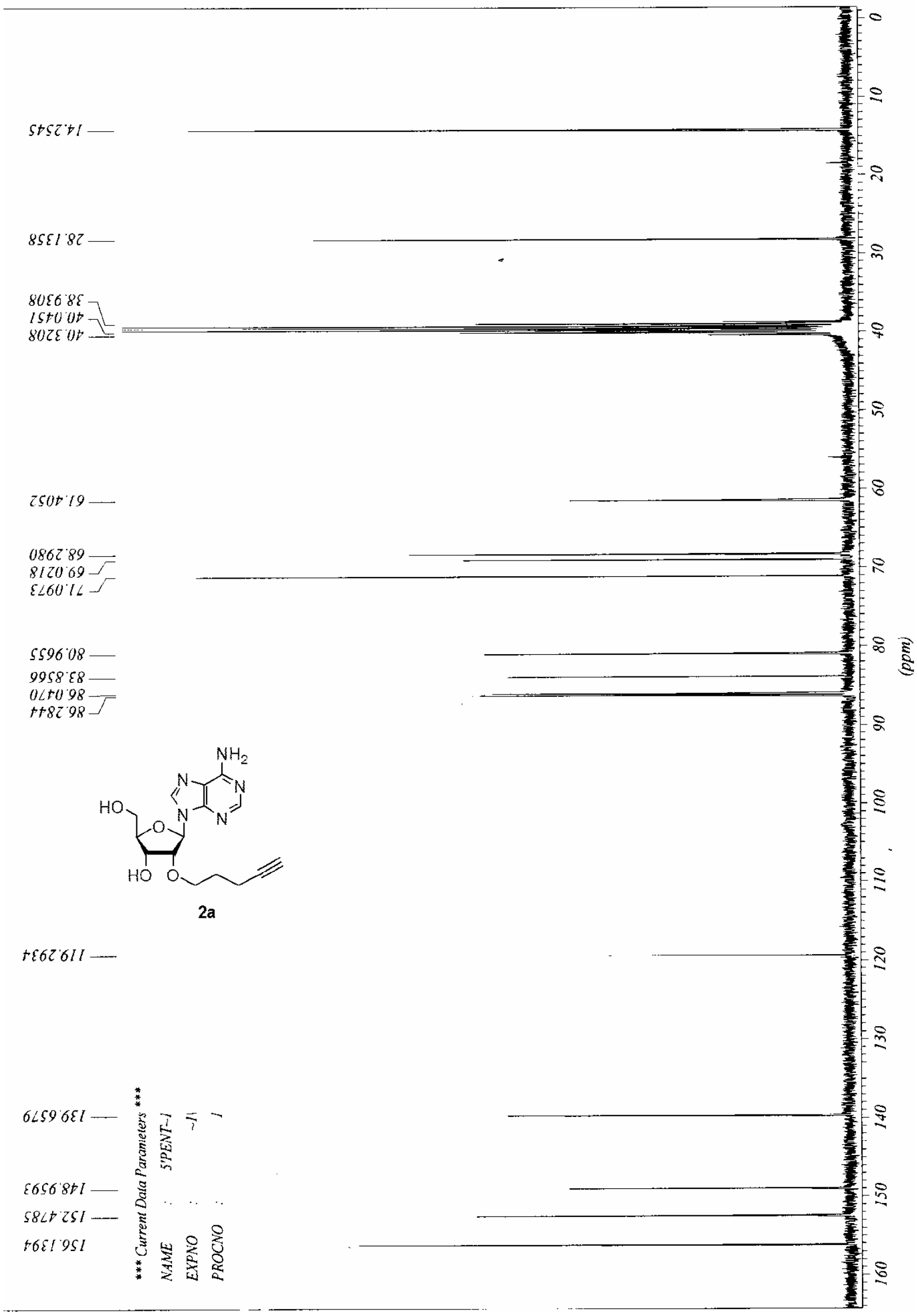


52091

58091

toE9 1

$+259 \%$

$\angle 190 \mathrm{Z}-\mathrm{H}$

$85 I 12-x$

$8+21 \mathrm{C}$

$8681 \mathrm{~T}-$

$88+1 / Z-L$

$1 \varepsilon \angle+Z-$

$16 \angle t C$

IS $8 t^{\circ} \mathrm{C}-$

$26+9.2$

$\angle \mathrm{tOE} \mathcal{E}$

$68901 \mathcal{E}$

$6 \operatorname{ICS} \varepsilon$

$00 \varepsilon 9 \varepsilon$

$0299^{\circ} \varepsilon$

$1 / 1 / 2$

$\mathcal{E E 8 6 : \mathcal { E } -}$

$\mathcal{E} \subseteq 66^{\circ} \mathcal{E}$

StZEt

$9 \angle \varepsilon \varepsilon+$

$\angle 28+5$

LOOS 5

$\angle L I S S^{\circ}$

त6E $\mathrm{S} S$

Eozz's-T

$\triangle E Z \subseteq]$

OZEI 9

$01519-$

$1805^{\circ} \mathrm{L}$

IIES $\angle-$ 辰

$\angle \angle S S \angle-$

$2509 \mathrm{~L}-\mathrm{A}$

$2629 \mathrm{~L}-$

$2+59 \mathrm{~L}$

$S 2108-$

$S \angle 100^{\circ}-$

$51508-F$

$182 \angle 8-$

$18+\angle 8$
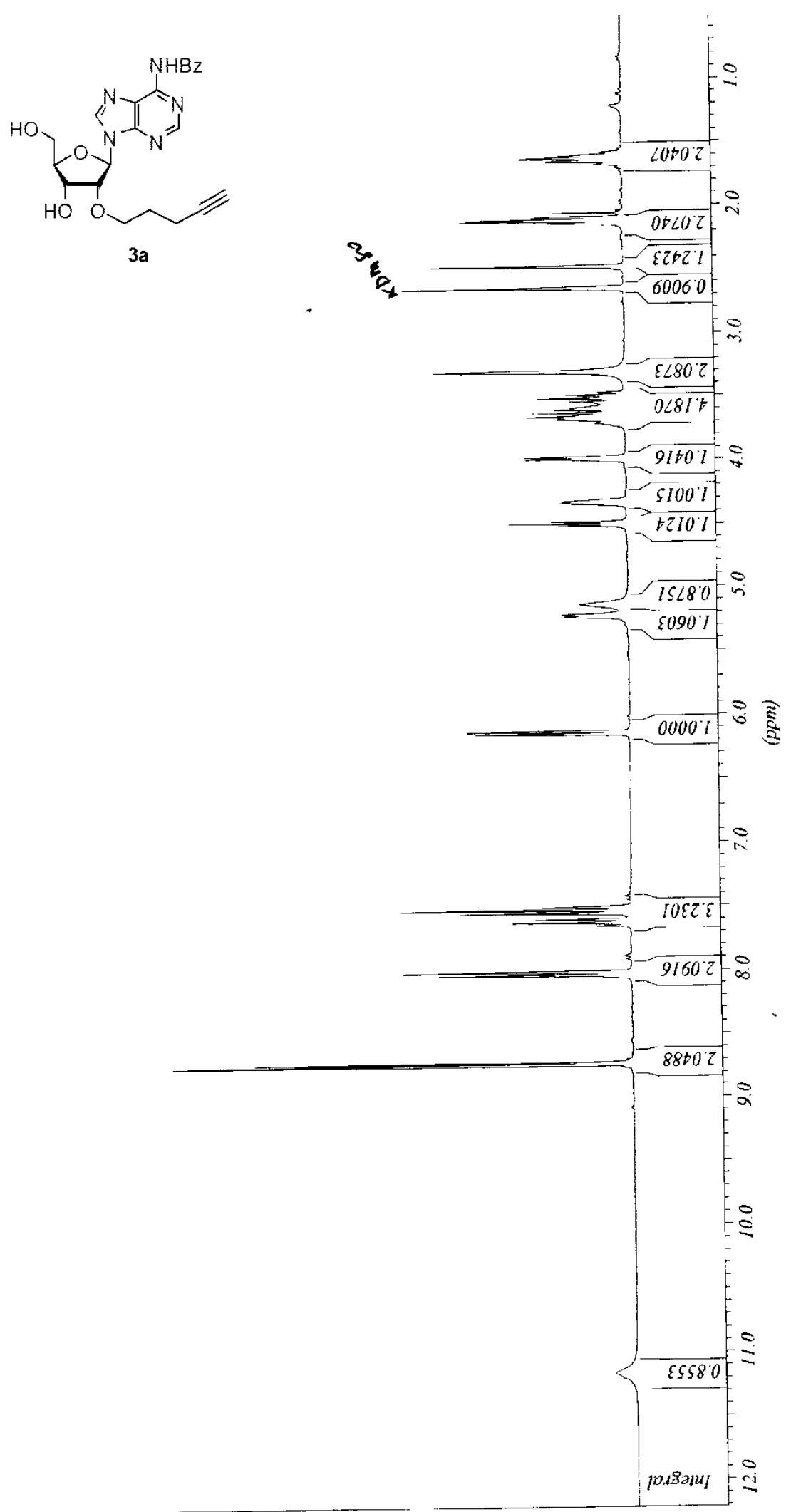

$0 \angle 9 I 11=$ 


$$
6 I I E+1-
$$

Et大) 82

$\$ 99988-$

I9EE $0{ }^{\circ}-$

948019

t10t 89

$6 \angle 2889 \div$

D29I'IL

ร6tI 18

$\angle E E 8.58-4$

t968.58

$0 \varepsilon 6098 \perp$

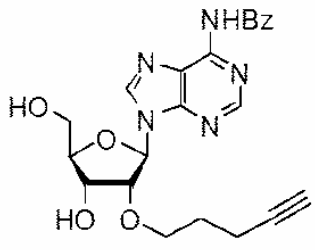

$3 a$

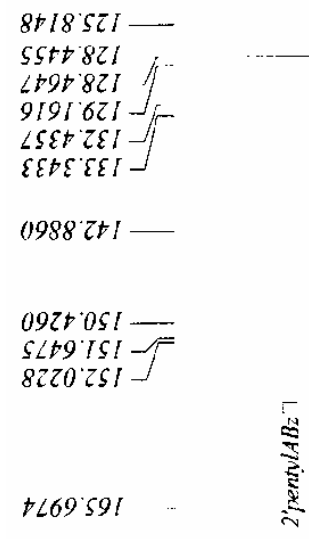




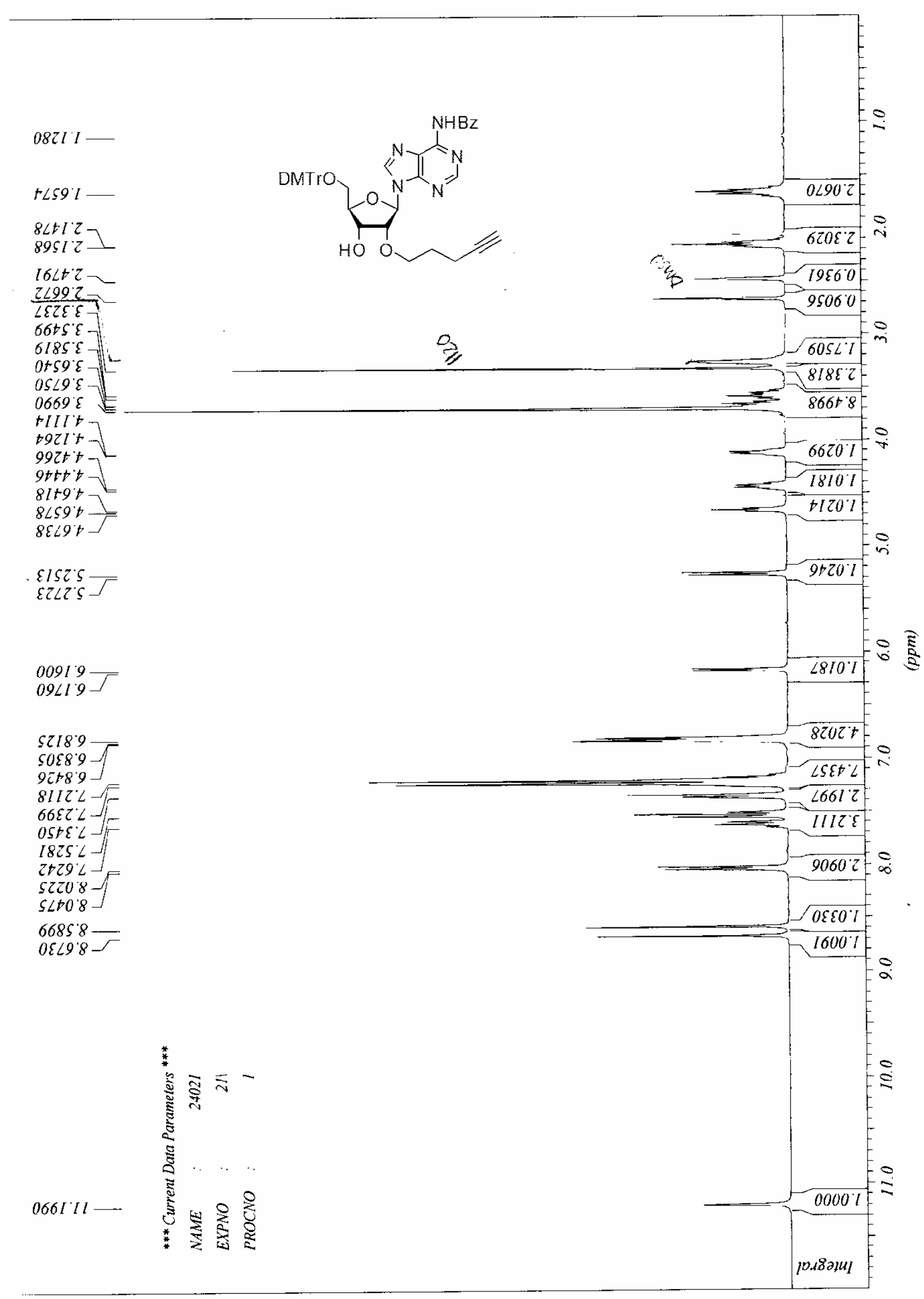




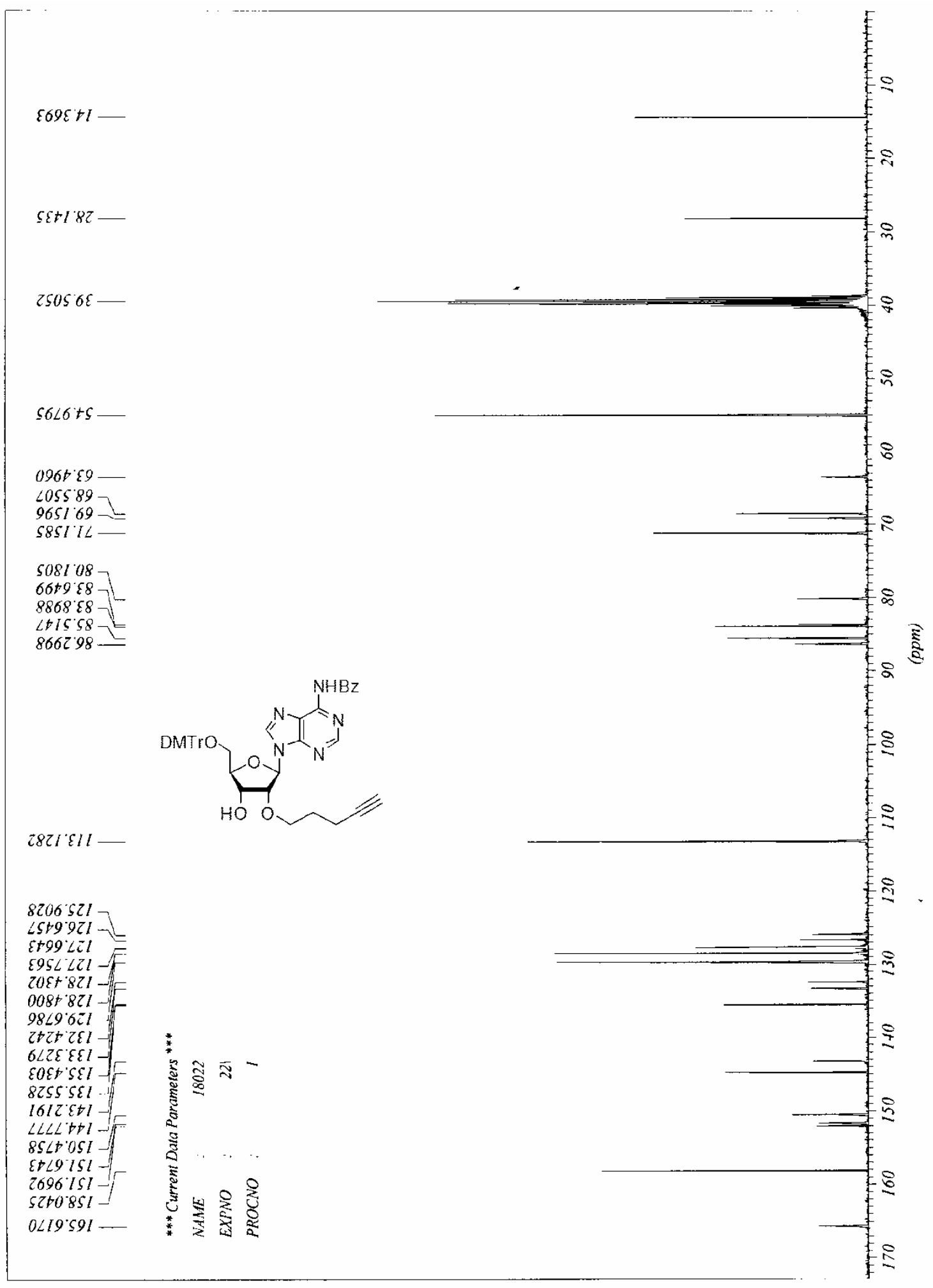




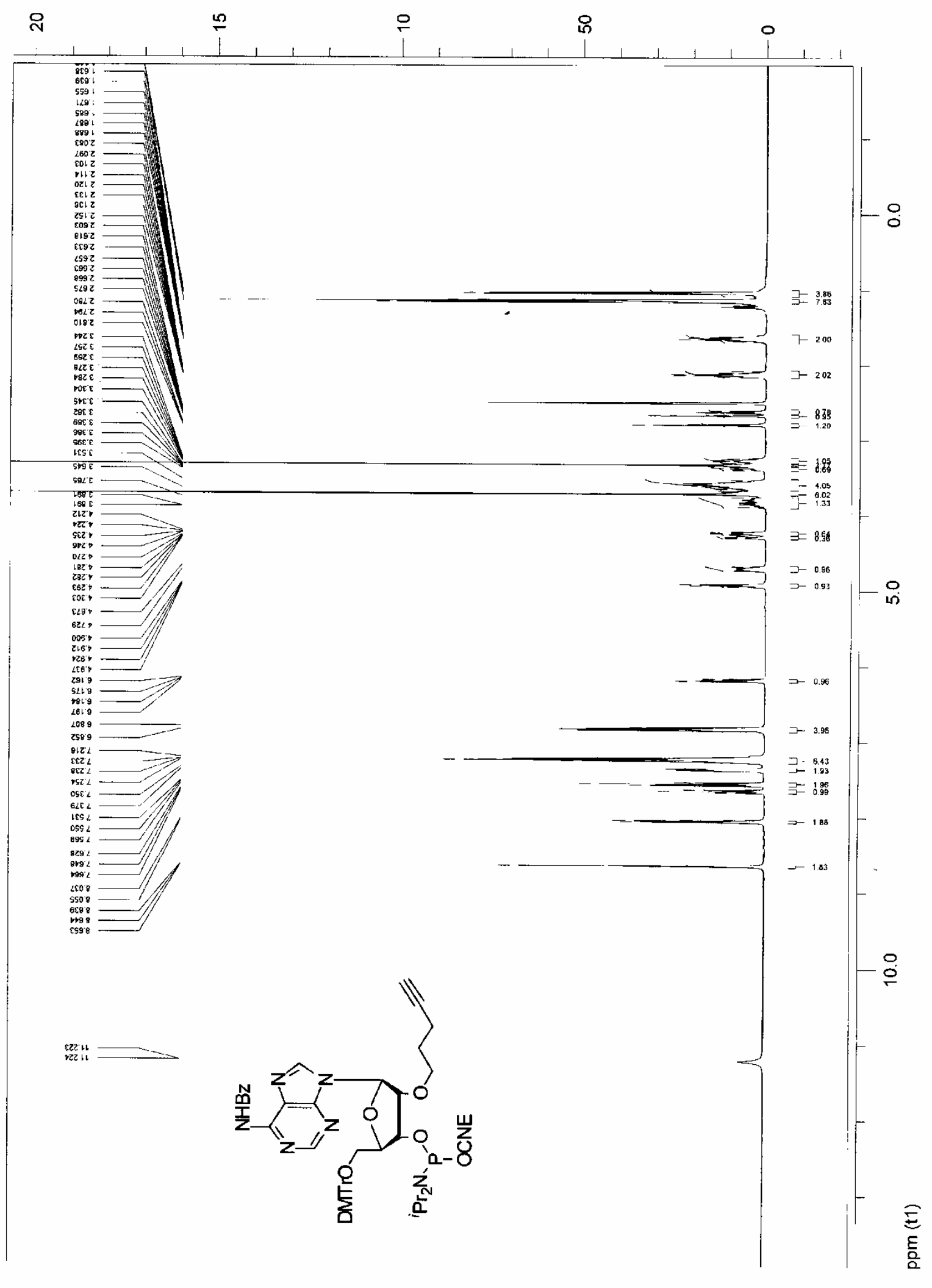




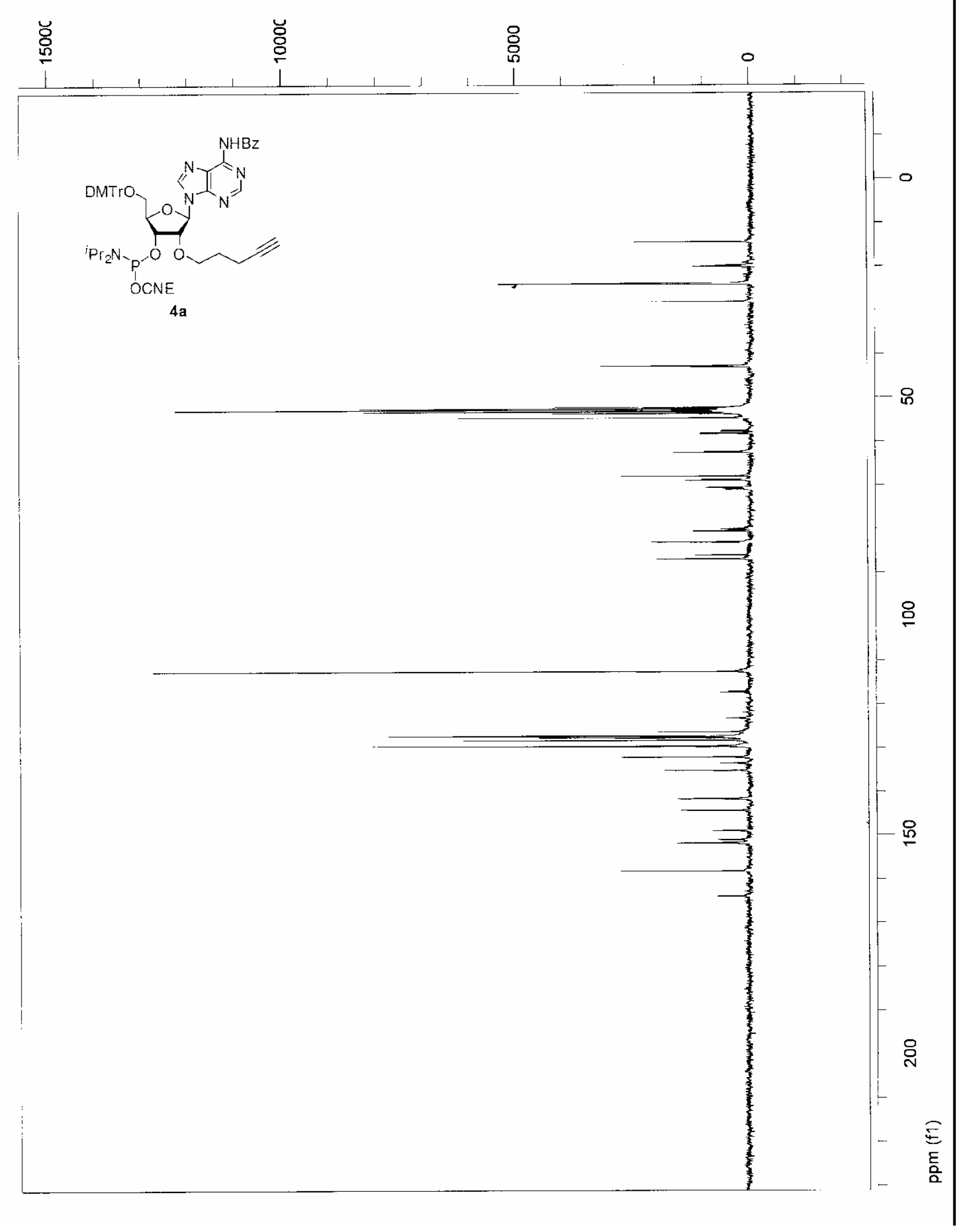




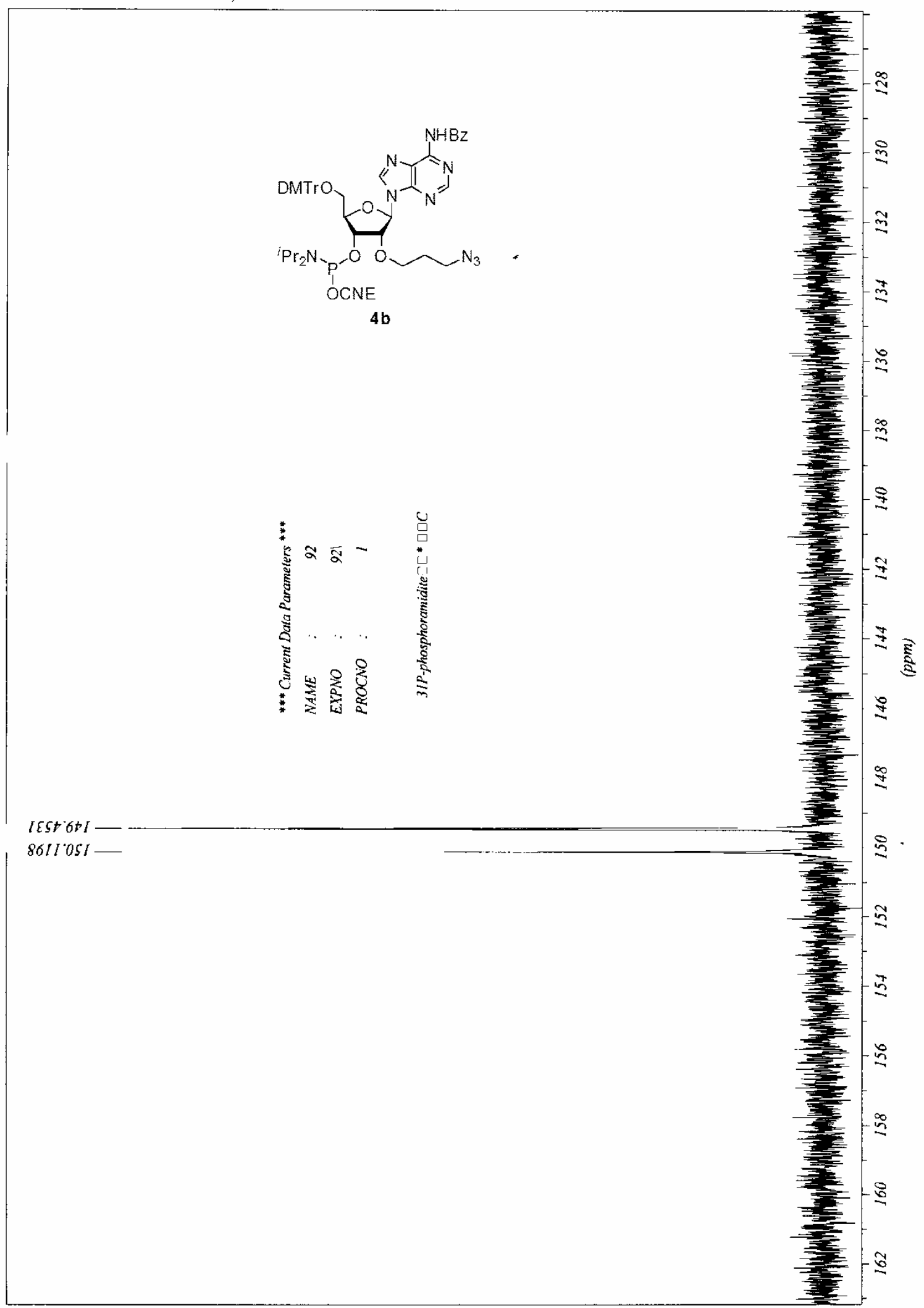




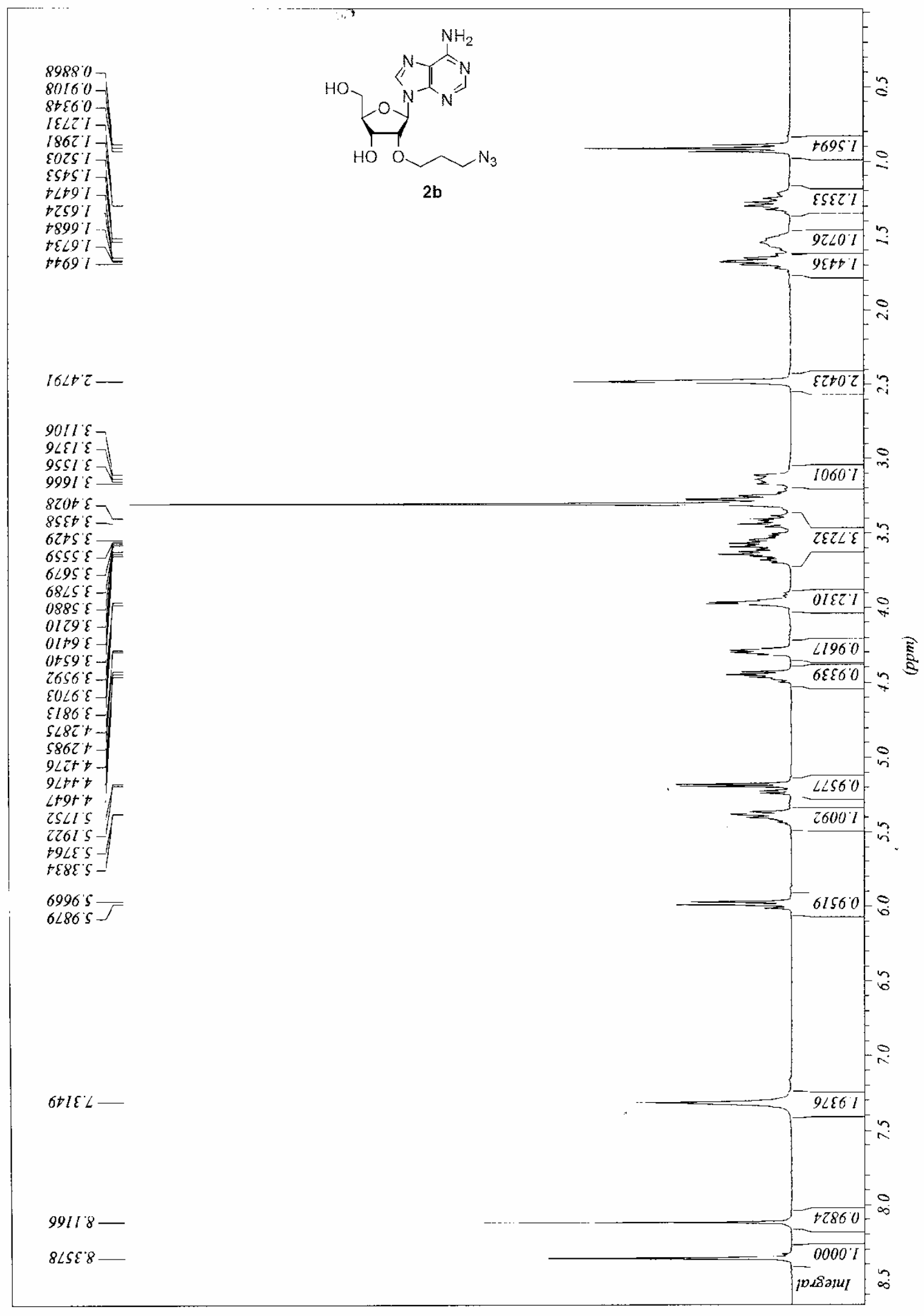




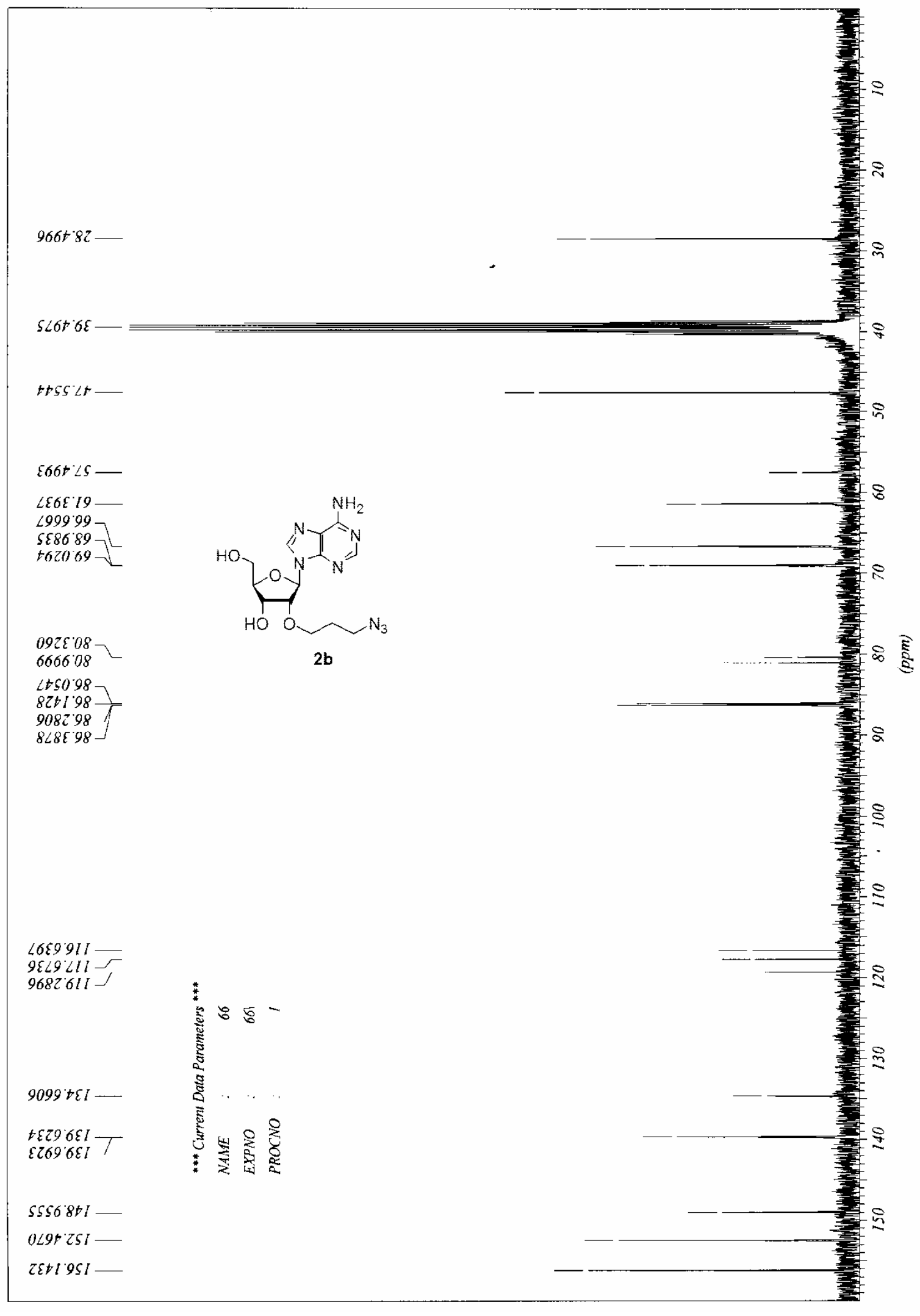




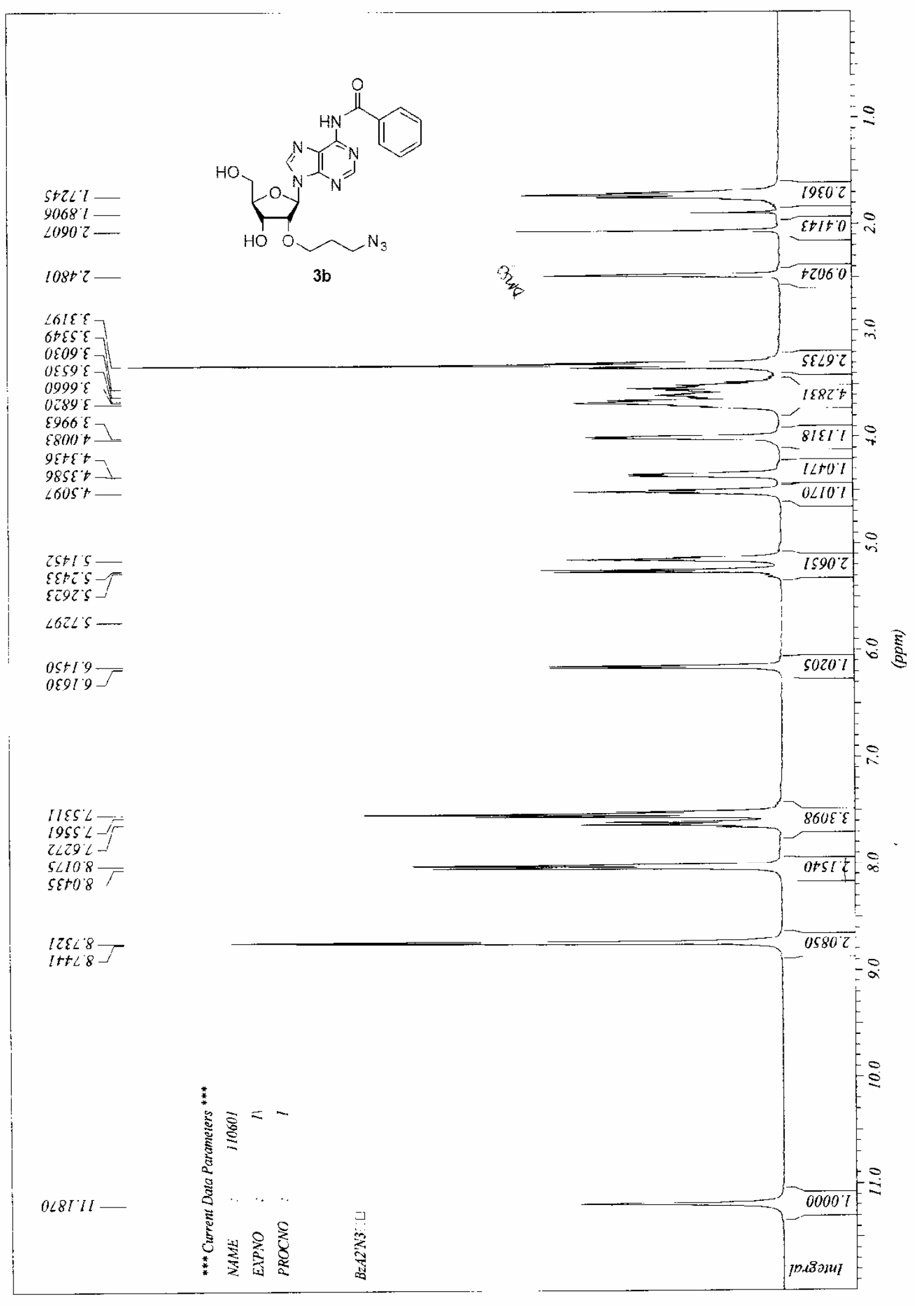




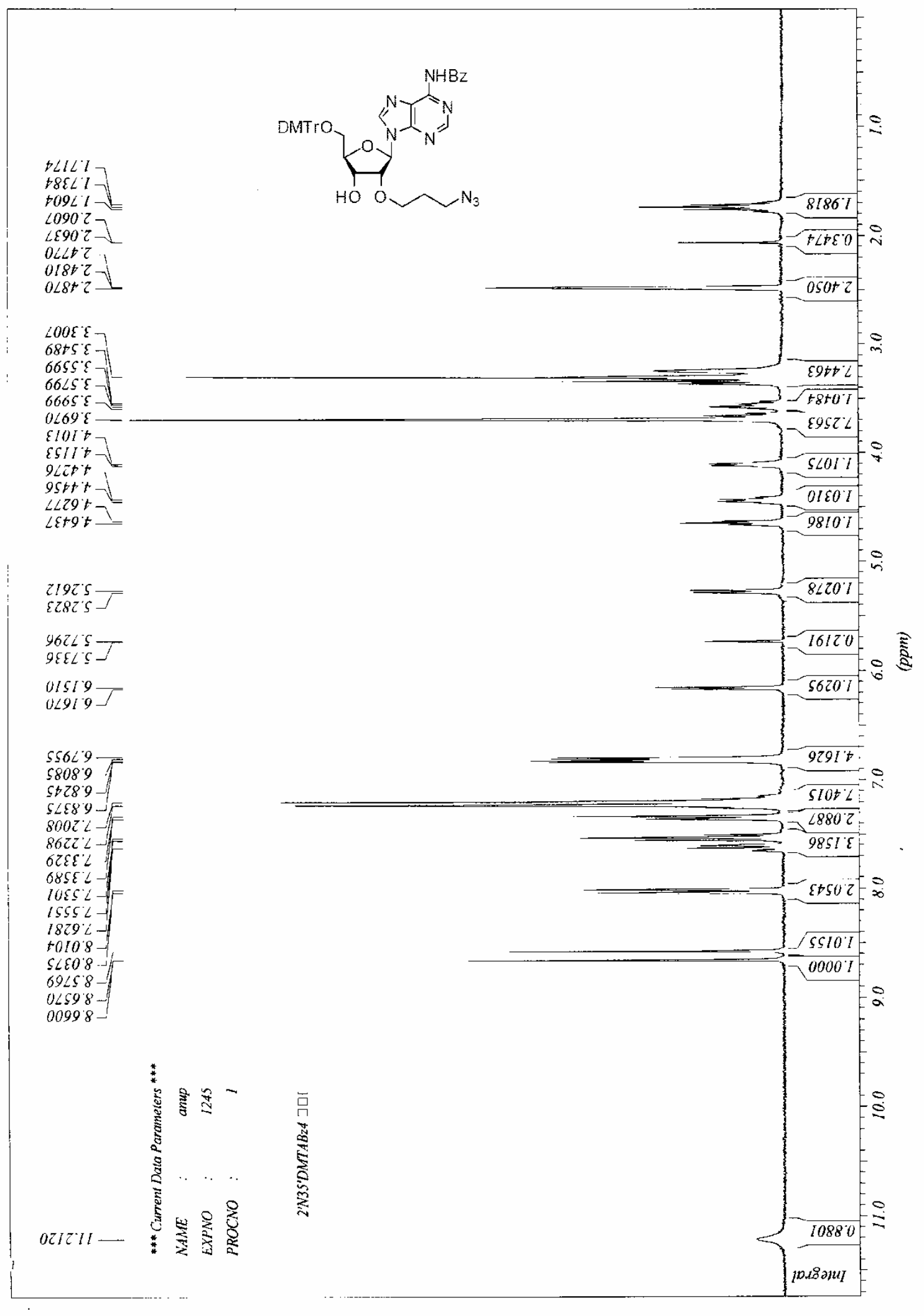




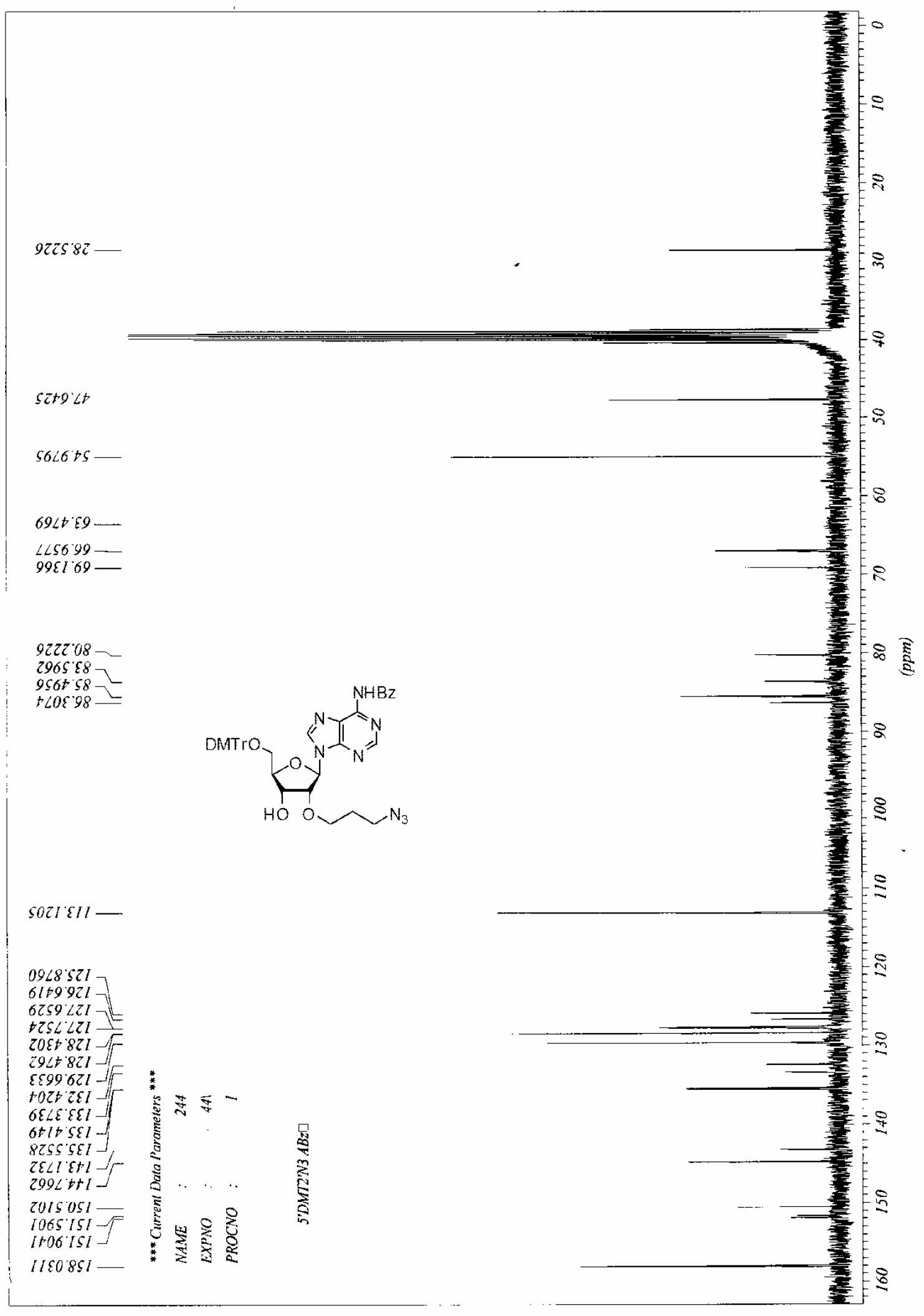




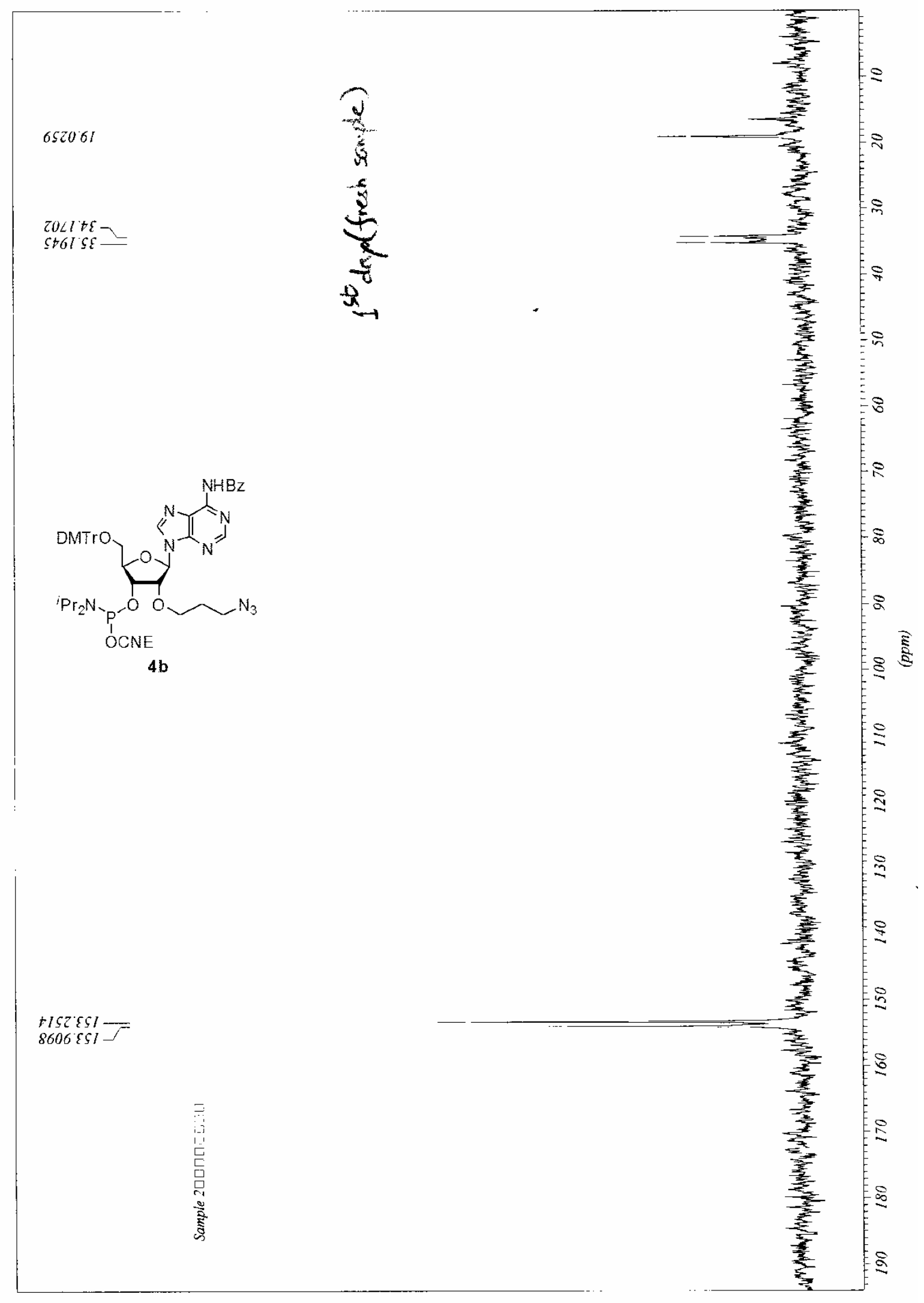




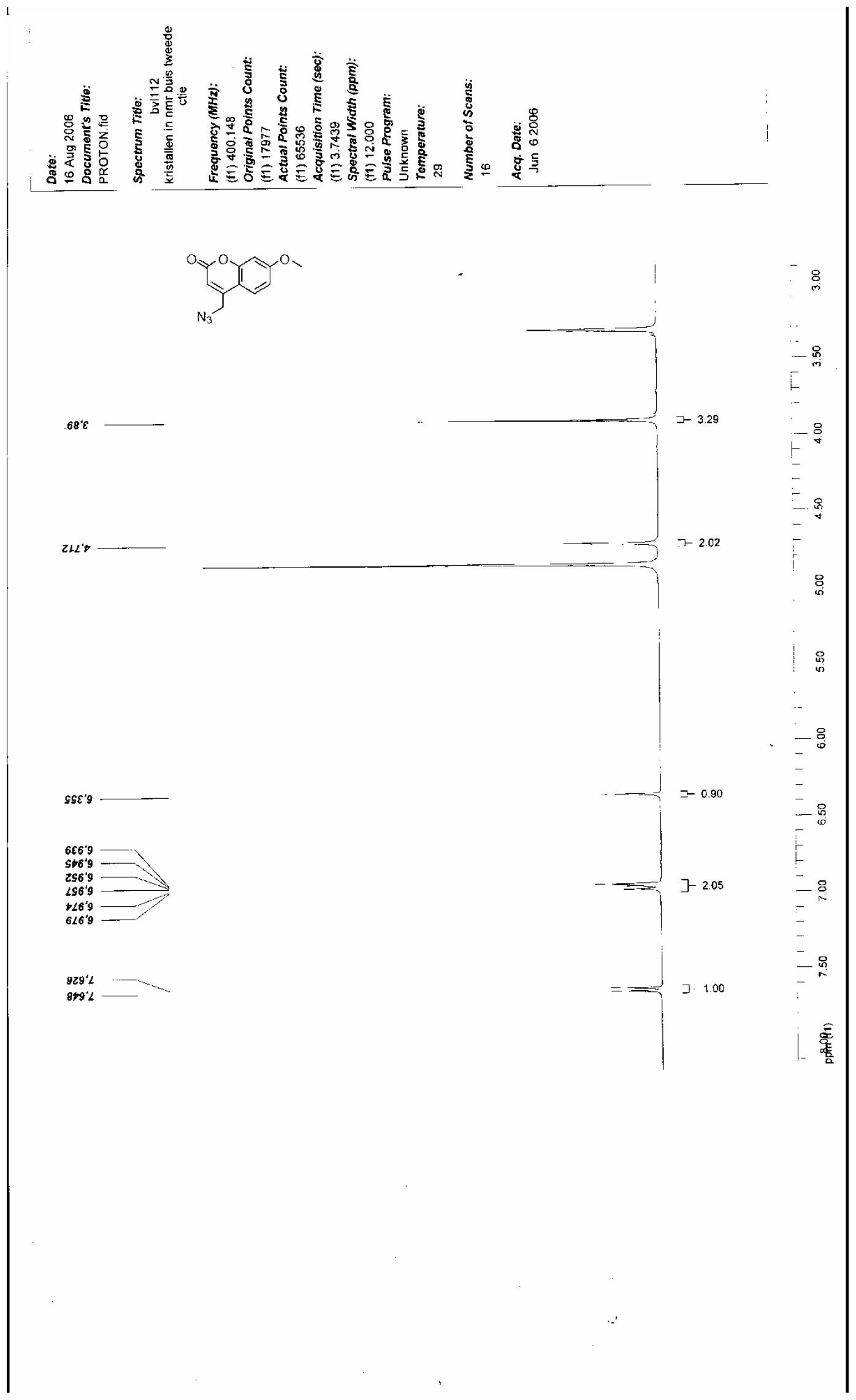




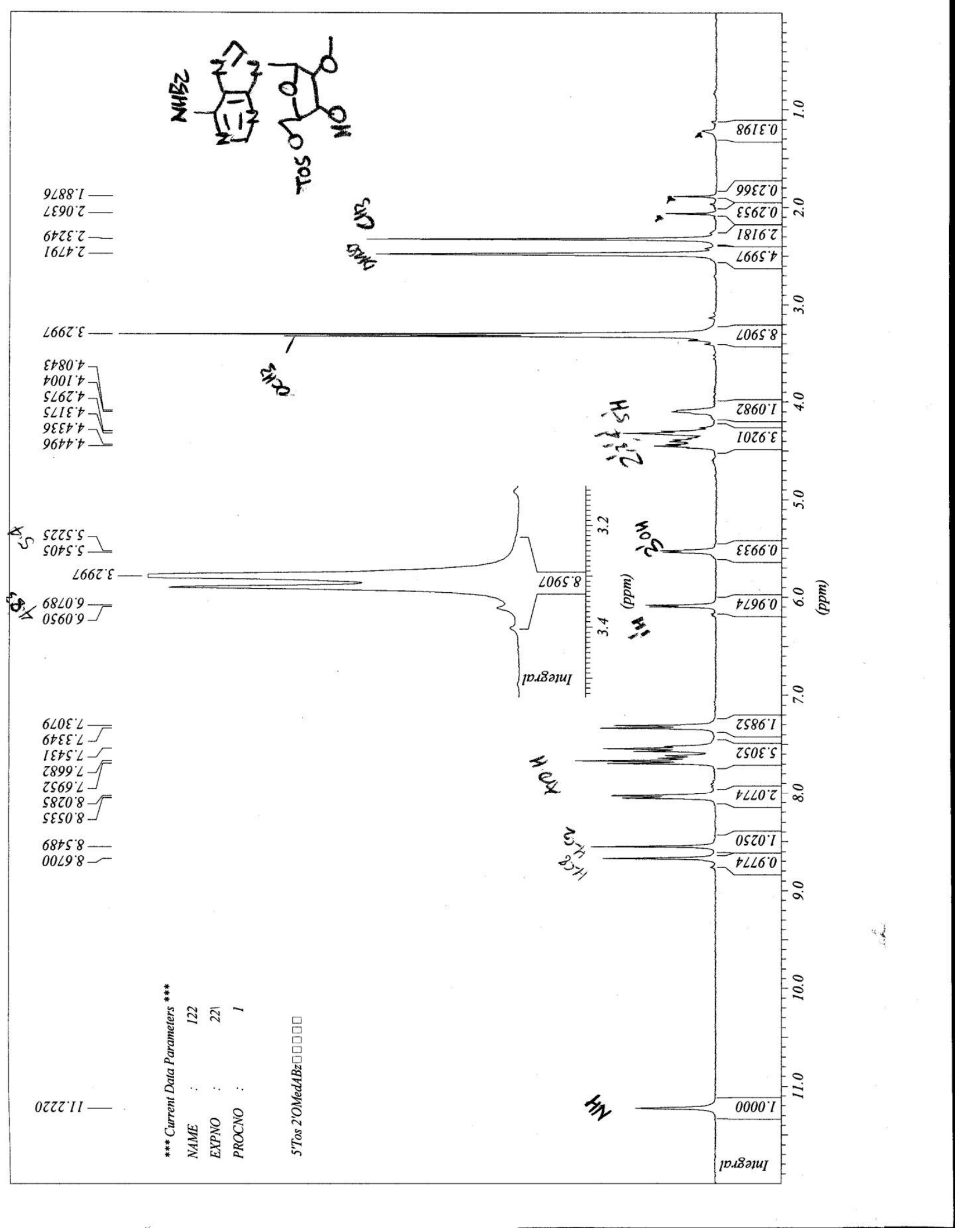




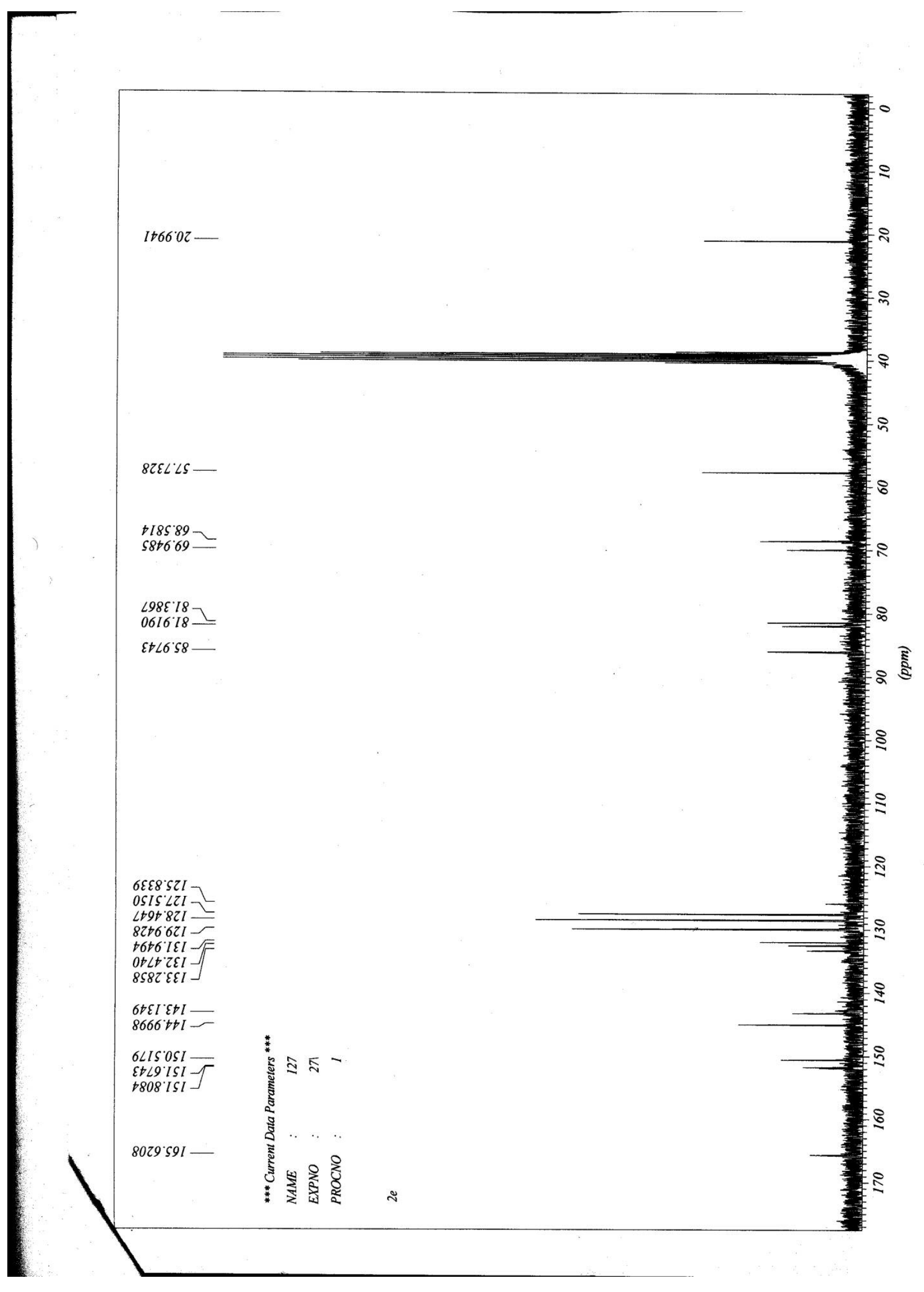




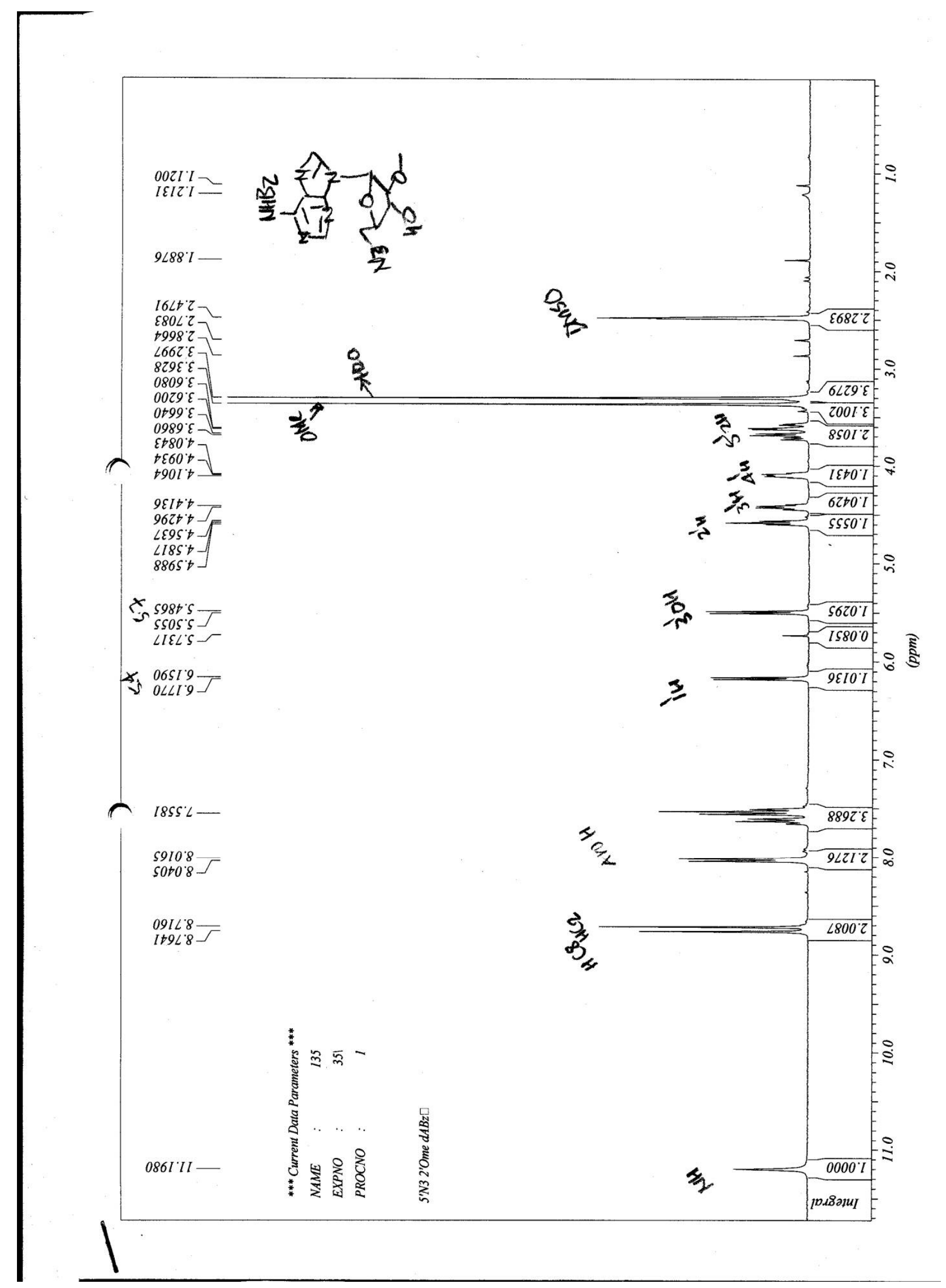




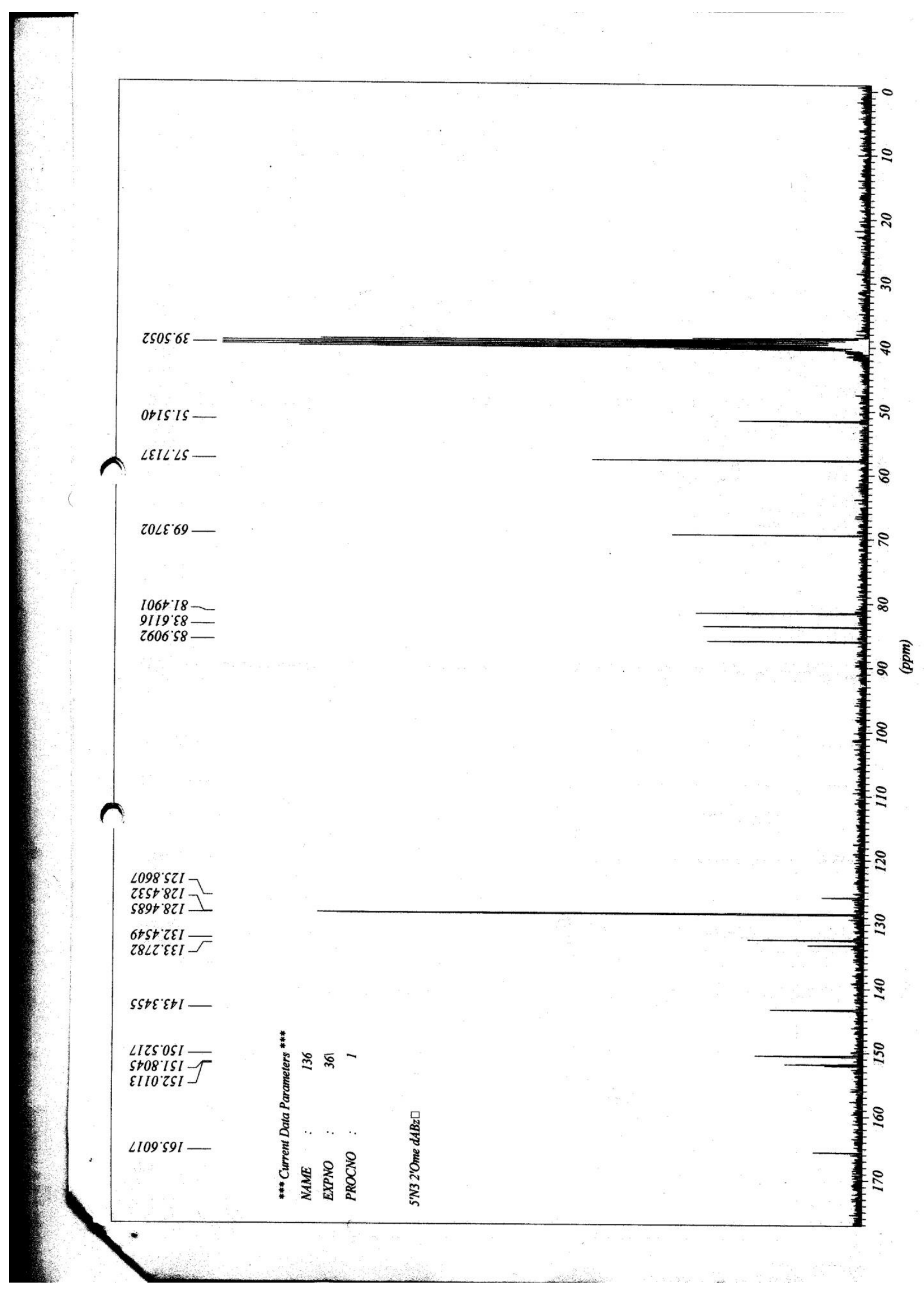




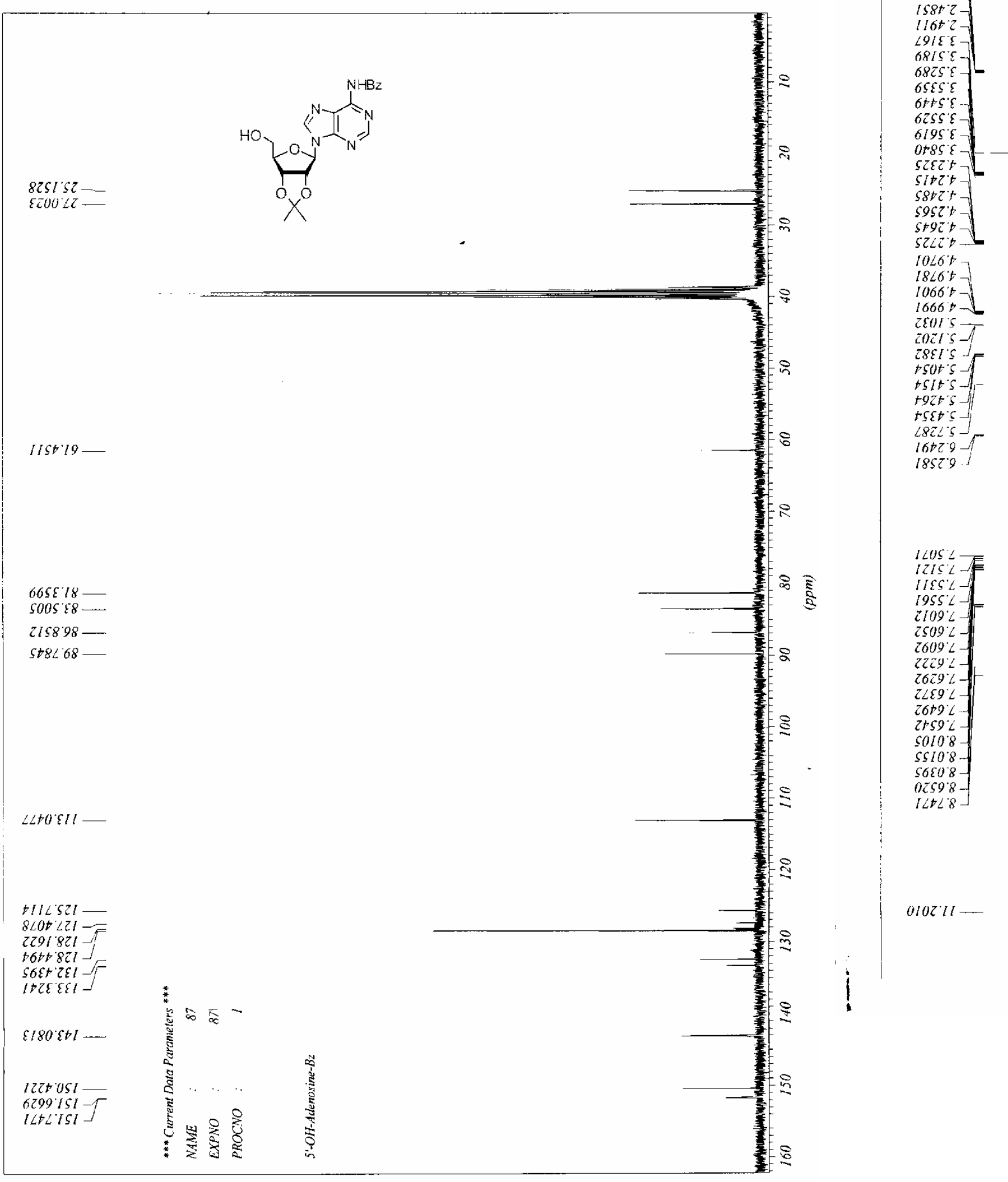




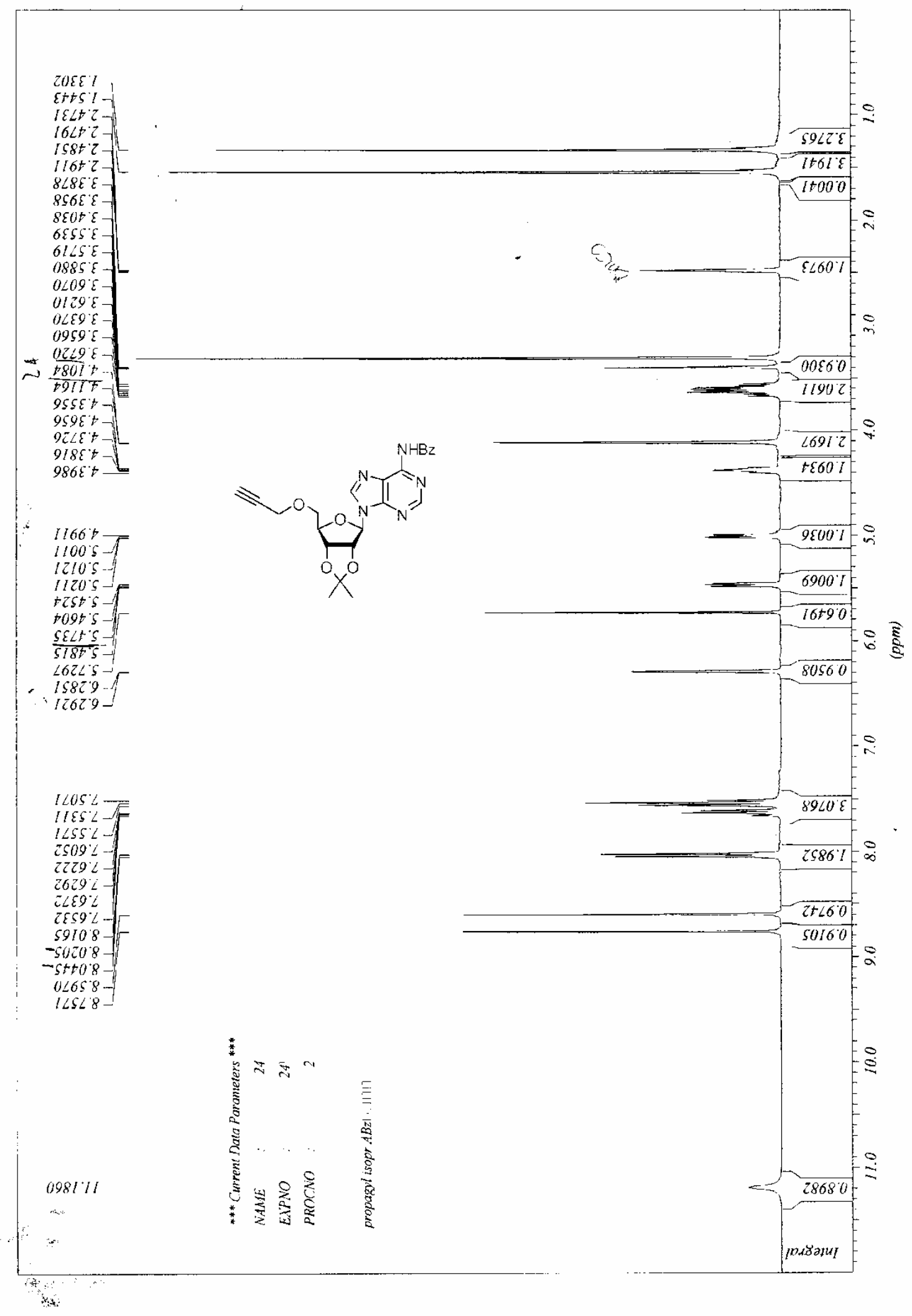




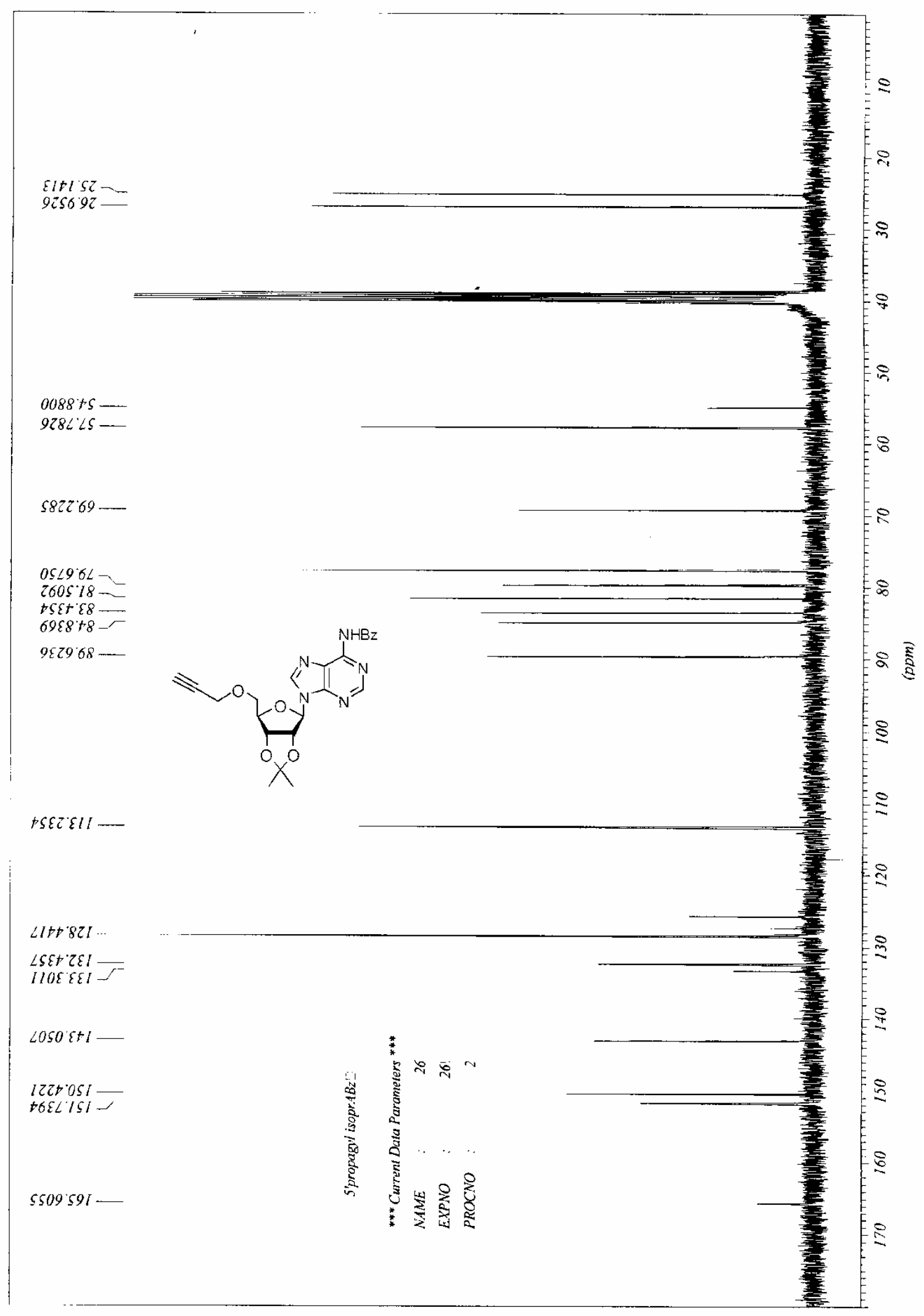




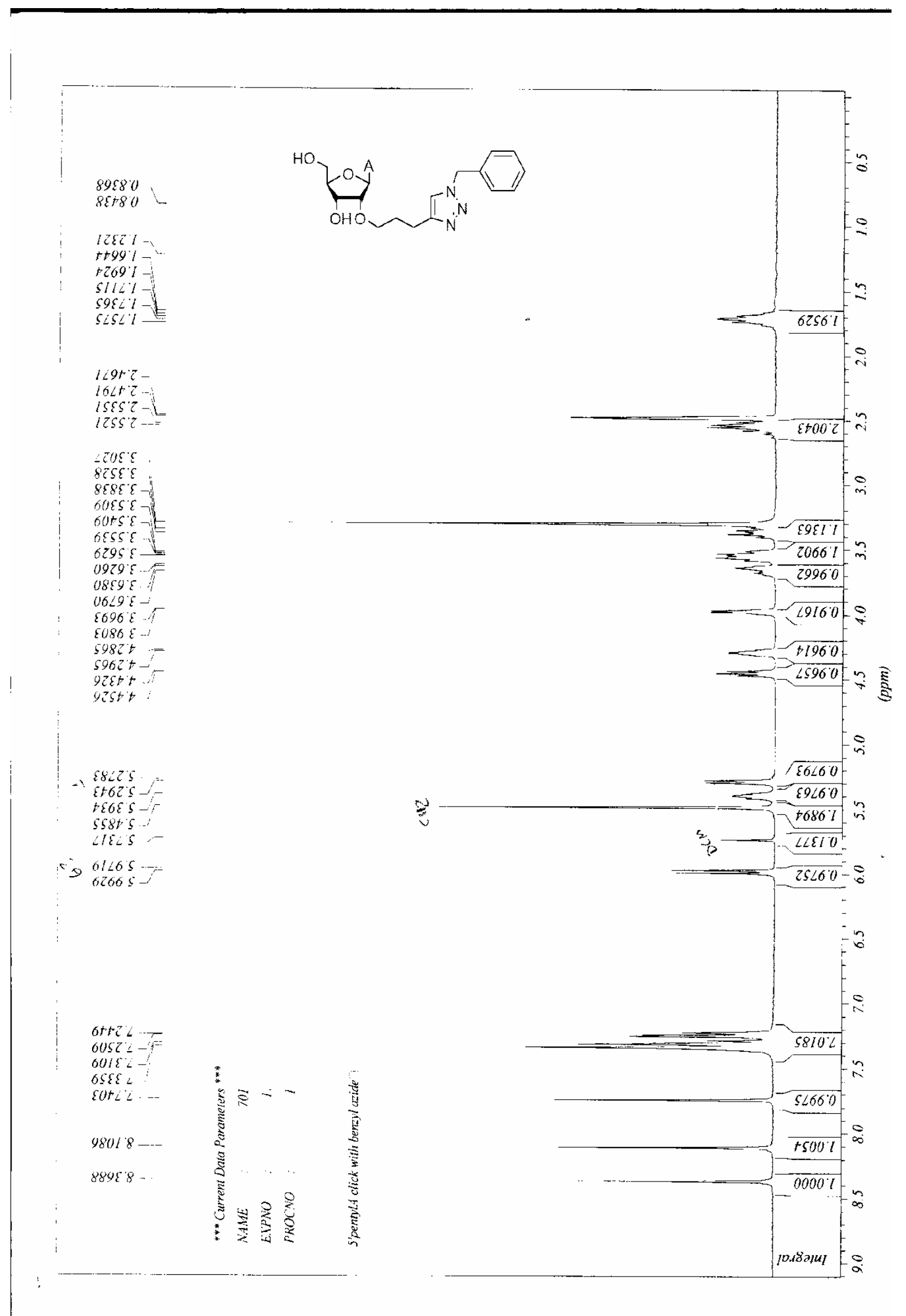




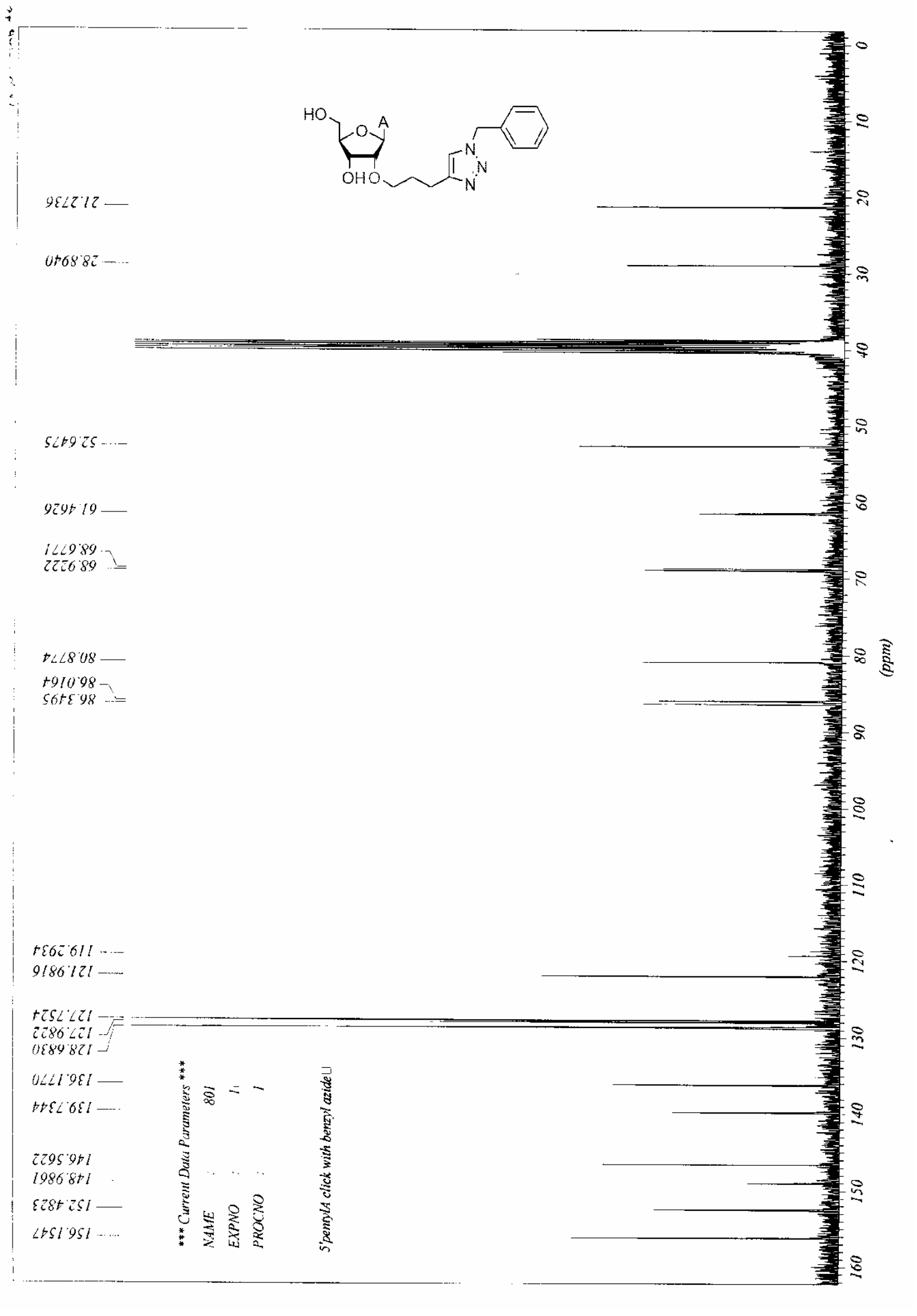




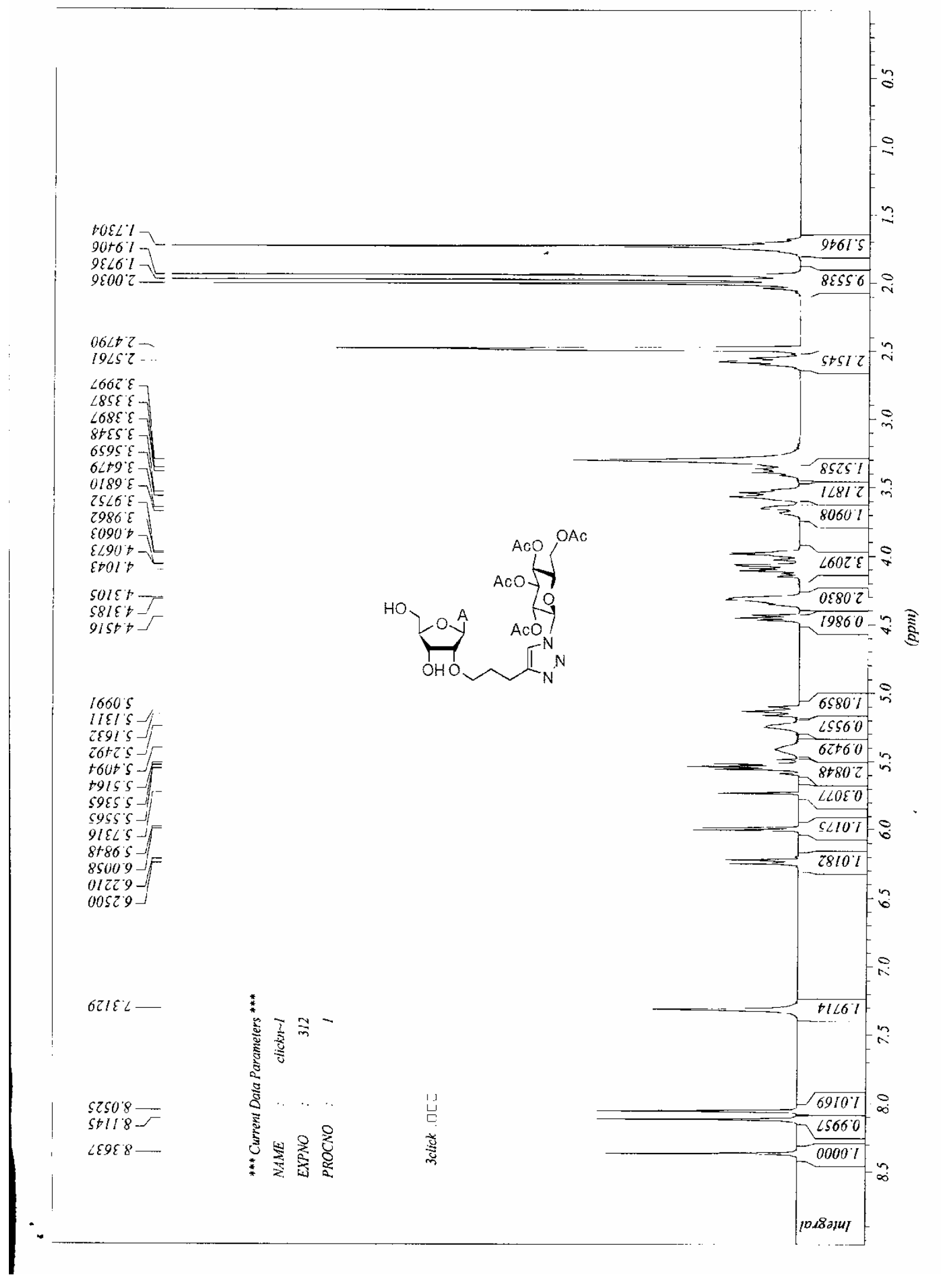


$866<61$

$9022^{\circ} 0 z$

9t5kOz

ILLFOZ

$826212=$

$5+6482$

$59998 \mathcal{8}$

$22+68 \varepsilon$

$81226 \varepsilon$

EIUS $6 \mathcal{E}$

$0 \angle L L 6 E$

I9EEO

968519

$159619 L$

829529

$\$ 69989 \ldots$

$509689-7$

$691102-F$

$6260 \mathrm{ZL} L$

$6891^{\circ} \mathrm{EL}$

$8+8608-$

$1889^{\circ} \mathrm{\varepsilon 8}-.-$

651198

2tEE.98
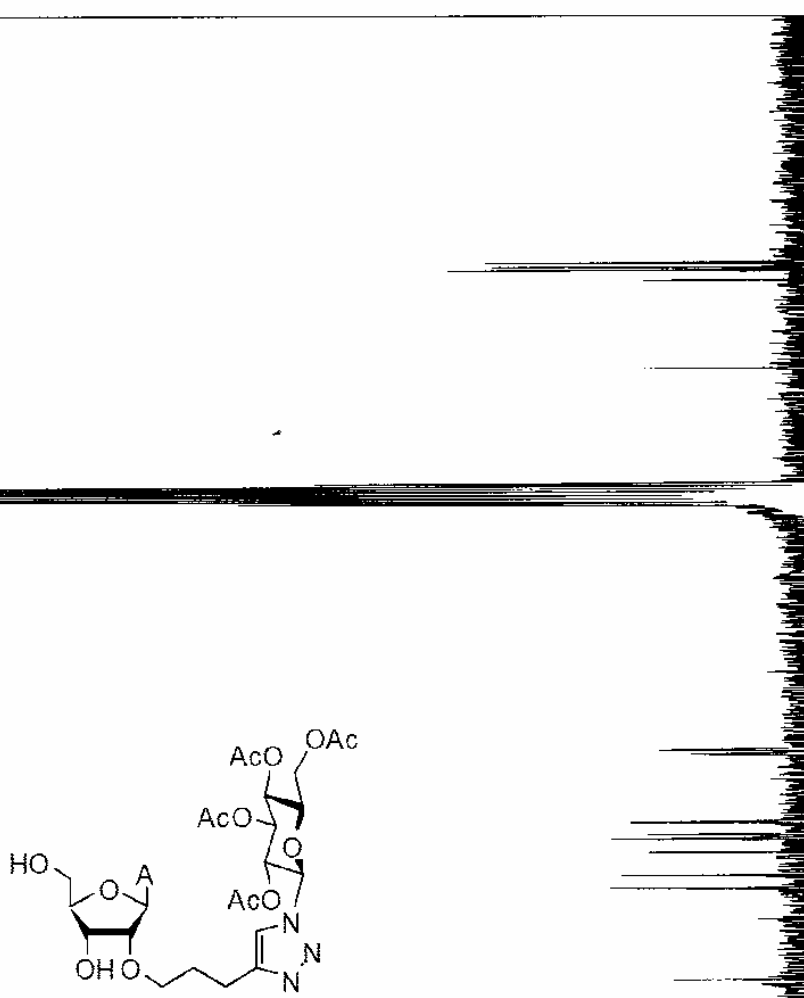

\%

in

$8558021=$

$\varepsilon \varsigma I \angle 6 \varepsilon l-$

$\angle 8669 \mathrm{Hl}$

$0 \angle 96801-$

$80 L t 2 S I$

$0 \angle H I 9 S I-$

$980+891-$

ESEE $691-$

$9 \angle 05691$

$+5000<1$

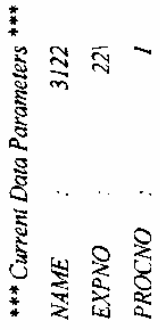

है 
SEtL' -

$\angle \angle S O Z$

$\angle S 90 Z$

$118 \mathrm{o}^{\prime} \mathrm{Z}$

$18 \angle S^{\circ} \mathrm{C}-2$

$z \varepsilon 6 \varsigma^{\circ} \tau-$

$\angle \mathfrak{E} 0 \mathcal{E}^{\circ} \mathcal{E}$

$860+6]$

$6955:$

$0 Z+9^{\circ} \mathcal{E}$

$0089^{\circ} \mathcal{E}$

$2888^{\circ} \mathcal{E}-$

$\varepsilon \angle \angle 6 \mathcal{E}$

$5862^{\circ}+$

95st' -

EOSZ'S-

$+\mathcal{E} 6 \varepsilon \mathcal{S}$...

$9<09 \mathrm{~S}$

$\angle 92 \angle S$...

$\angle E E S S$ -

$67 \angle 6,5$

62665

96569

$\angle 8866^{\circ}-F$

$\angle 2102-$

$\angle 020 \mathrm{~L}$

$681 \varepsilon^{\circ} \mathrm{L}$

$20024=$

EIEL $L$

$16 \angle 8 \mathrm{~L}-$

95018

8598: 8

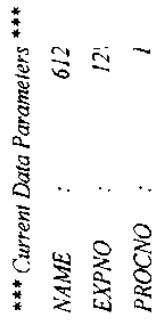<smiles>COc1ccc2c(CN3C=C(CCCOC4C5CCC(O5)C4O)NN3)cc(=O)oc2c1</smiles>
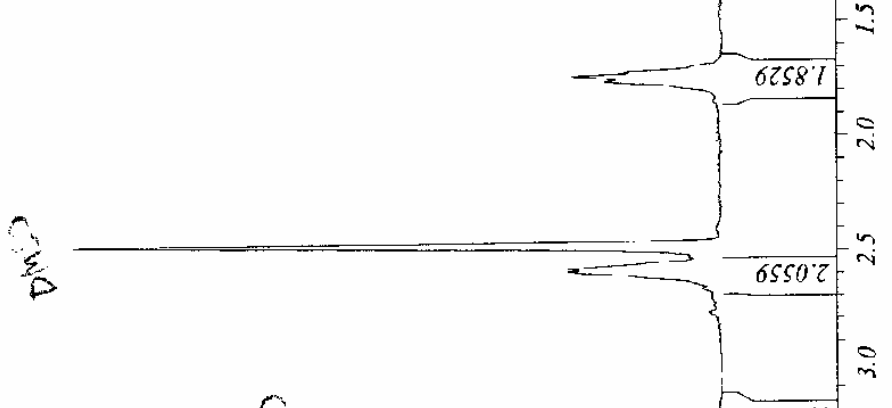

3

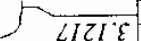
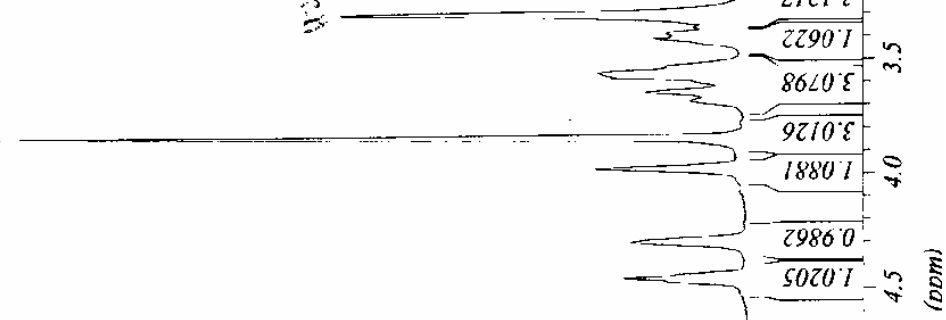

년 웡

, 


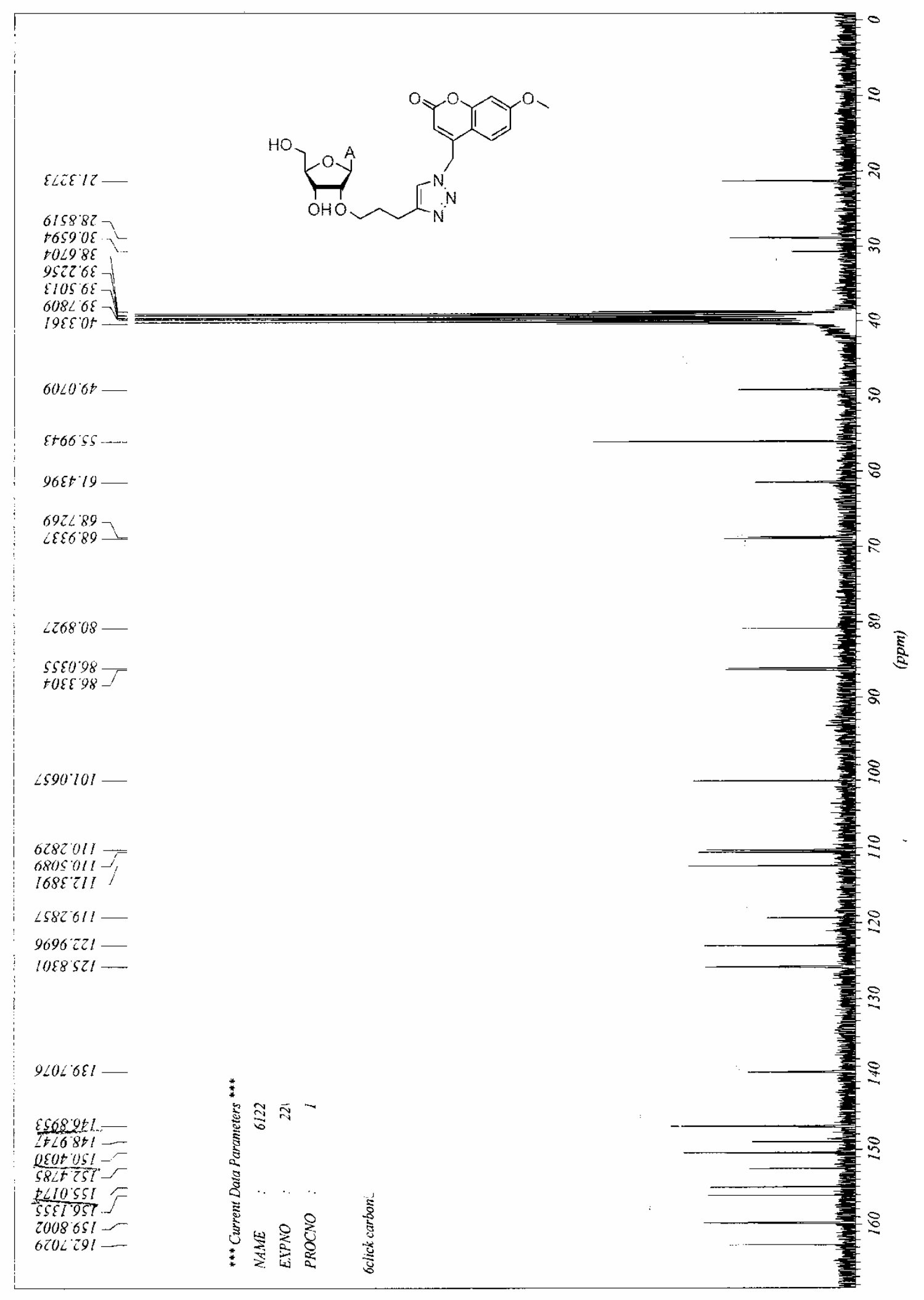




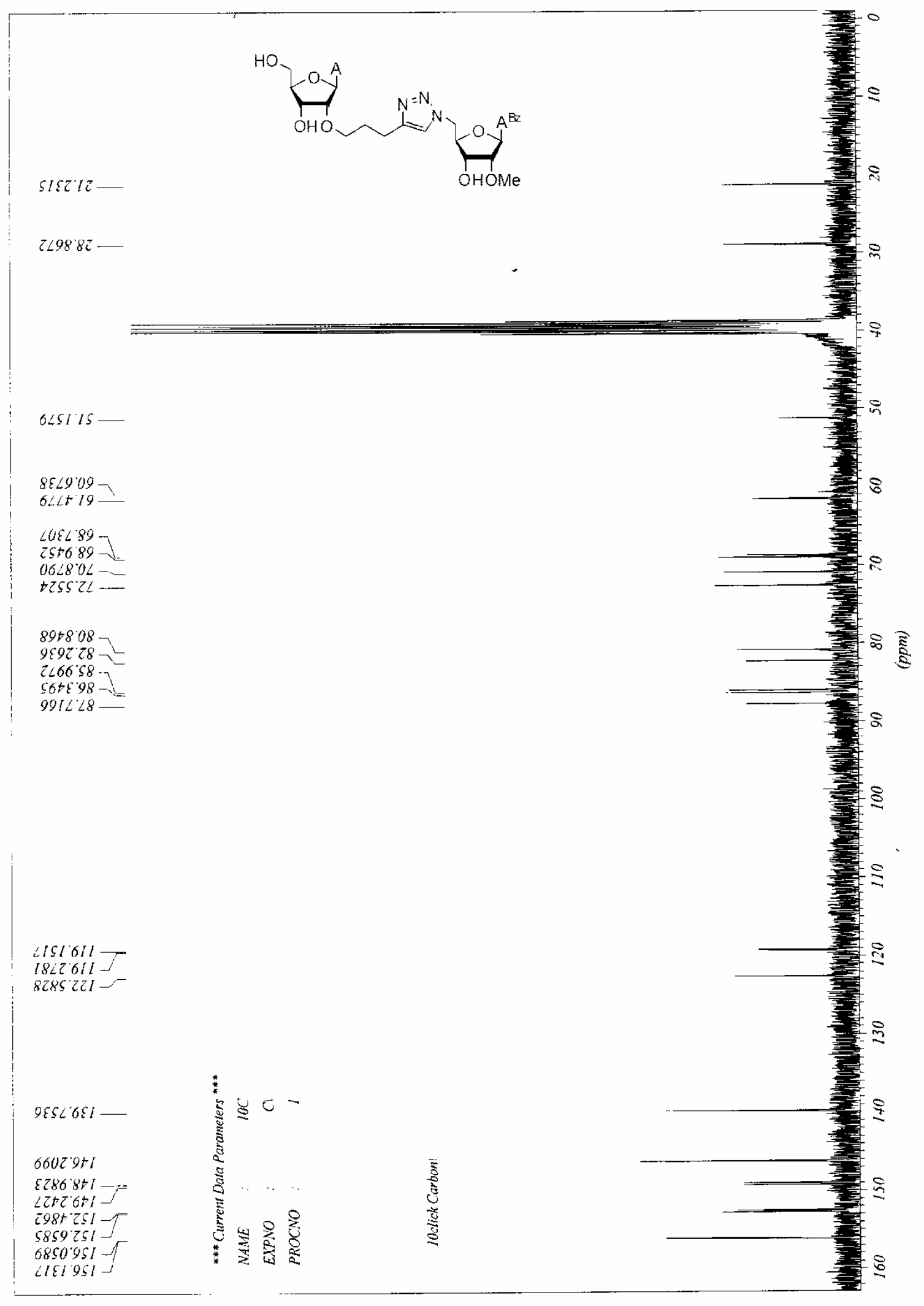




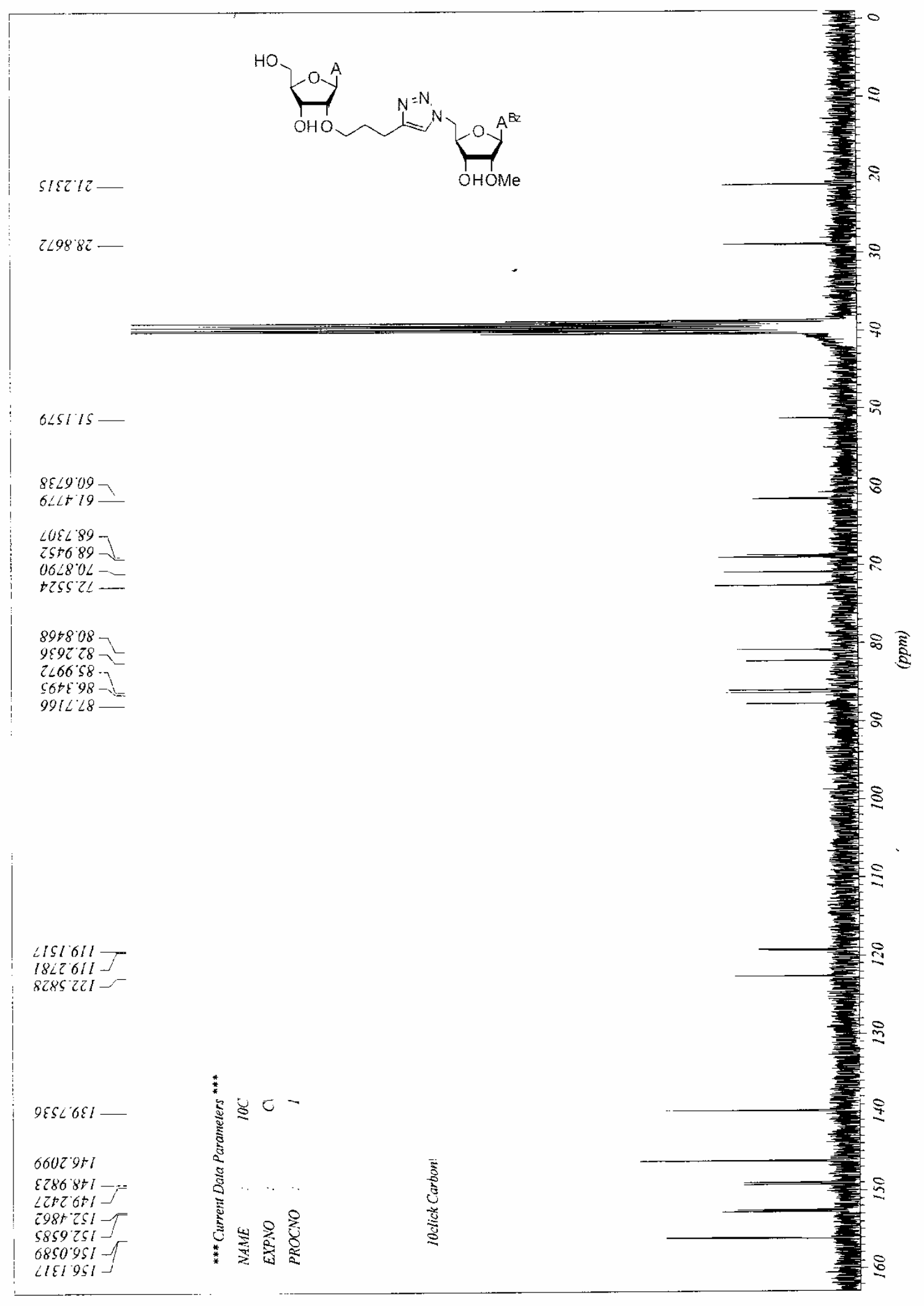




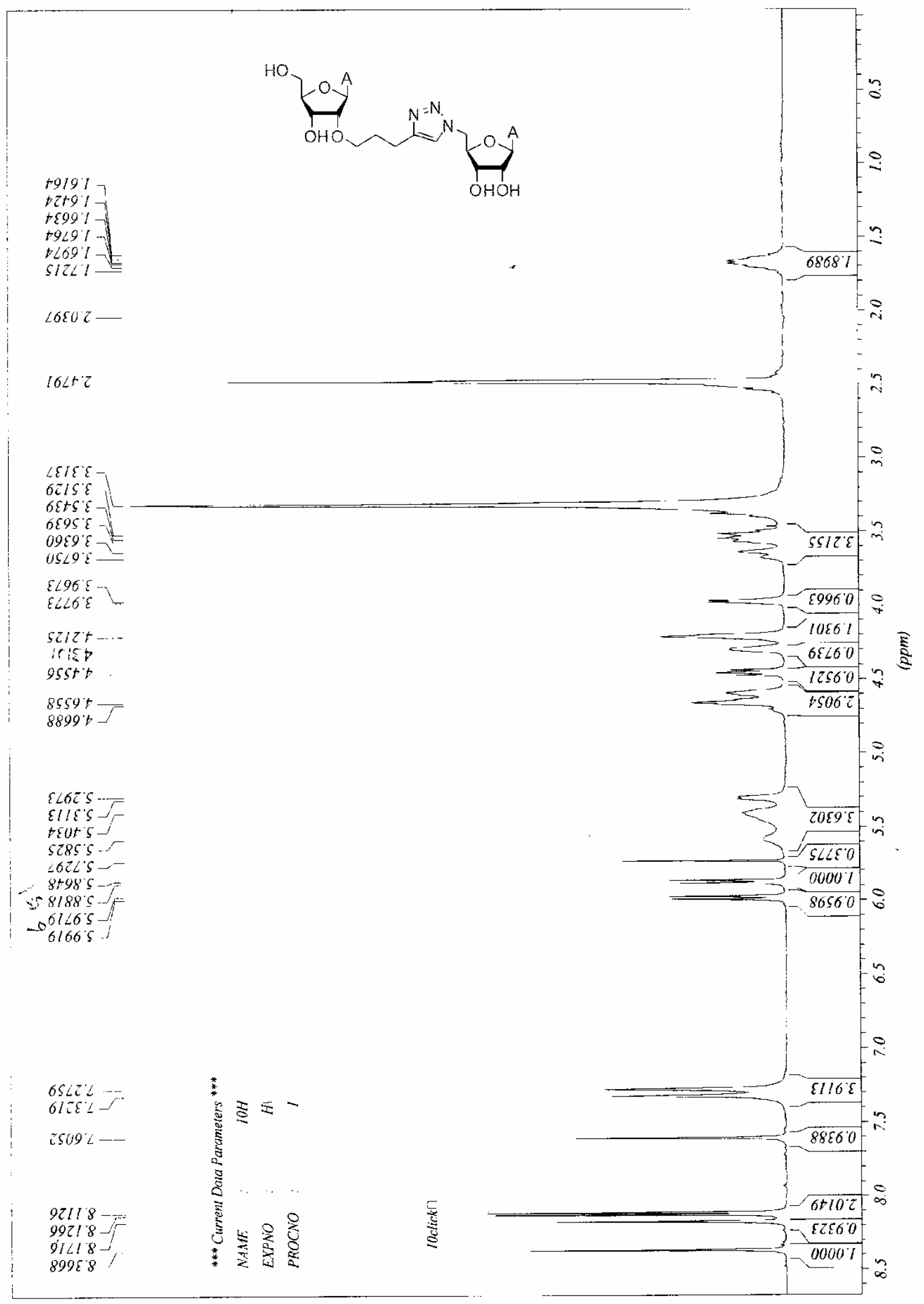




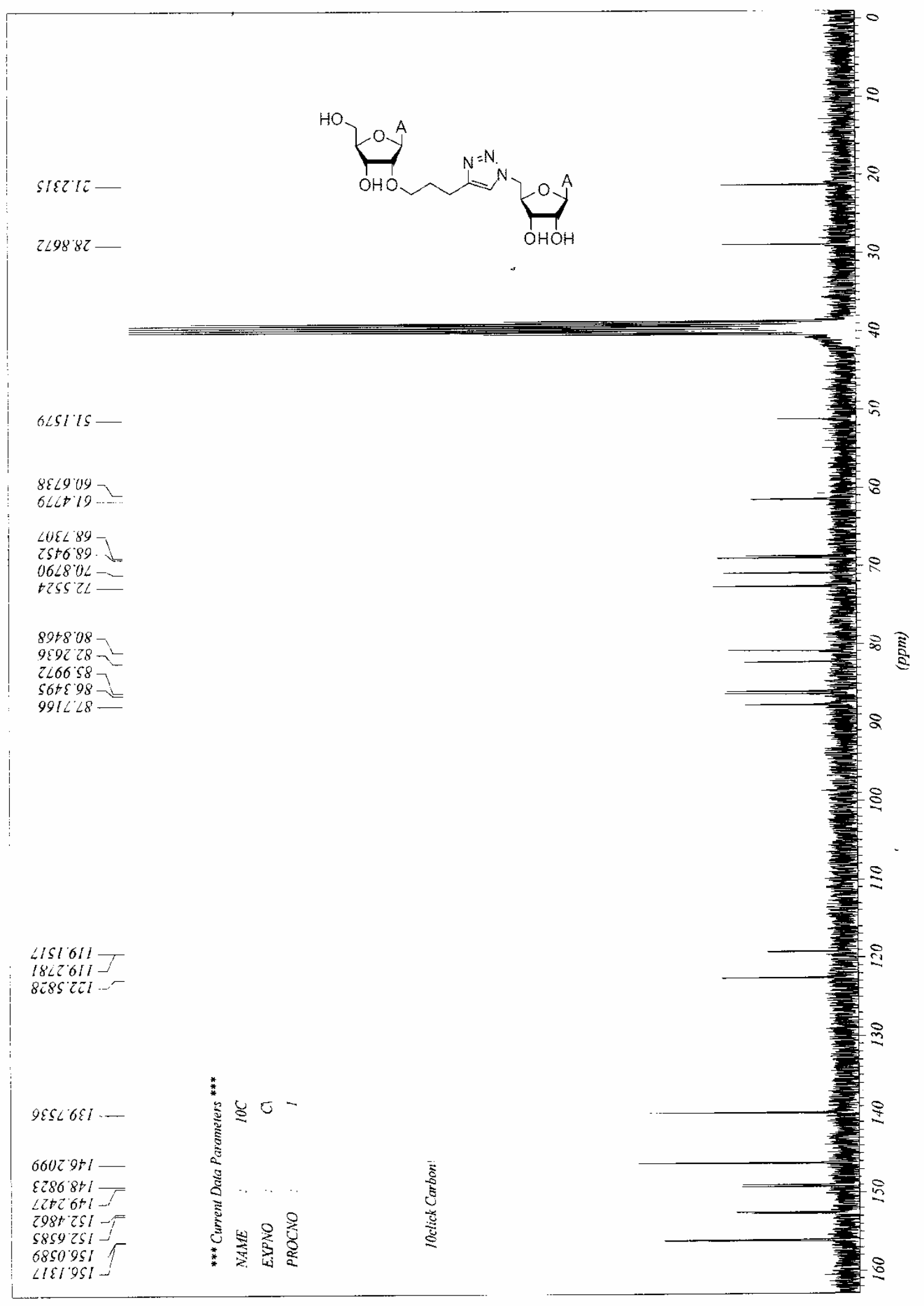




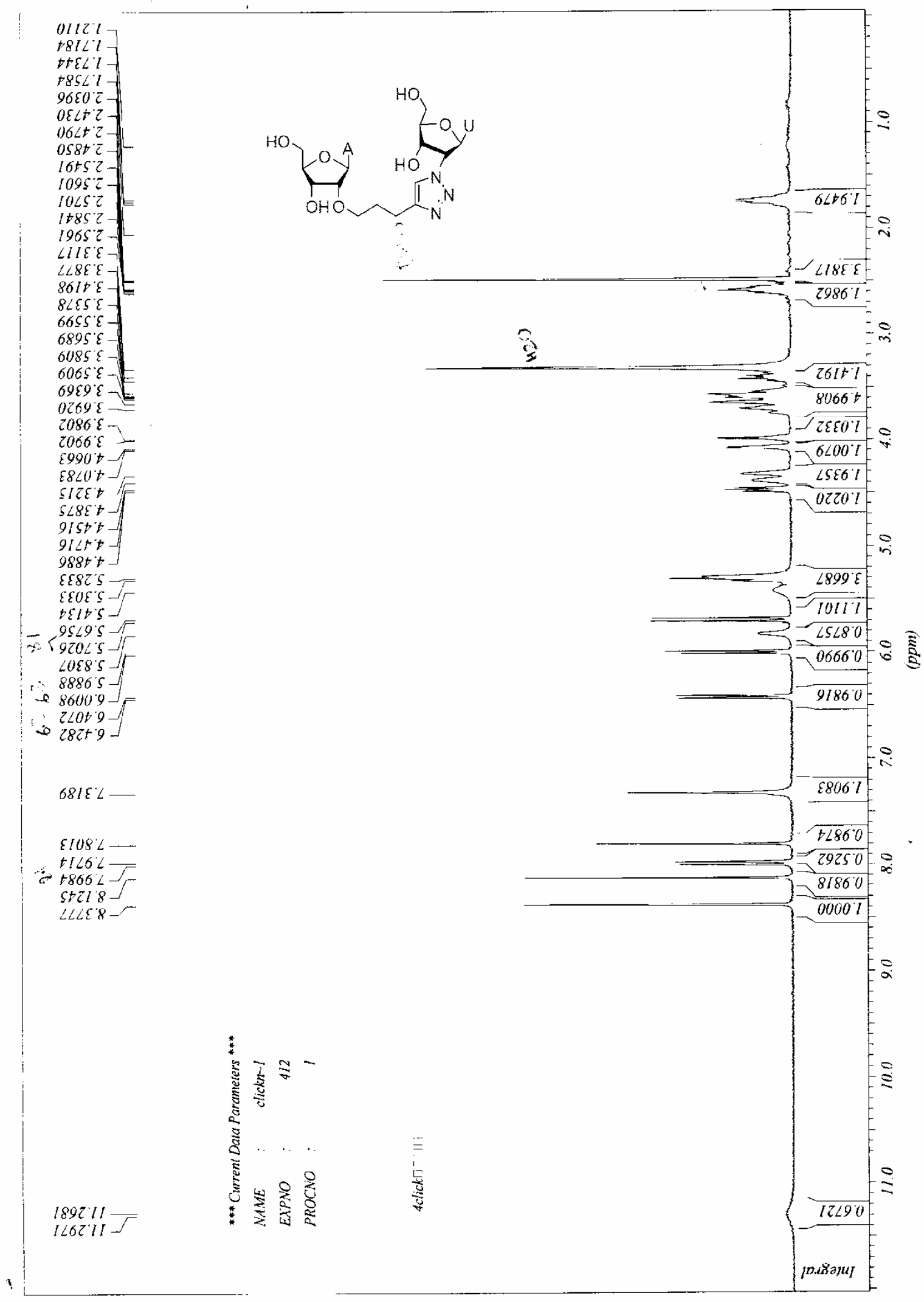




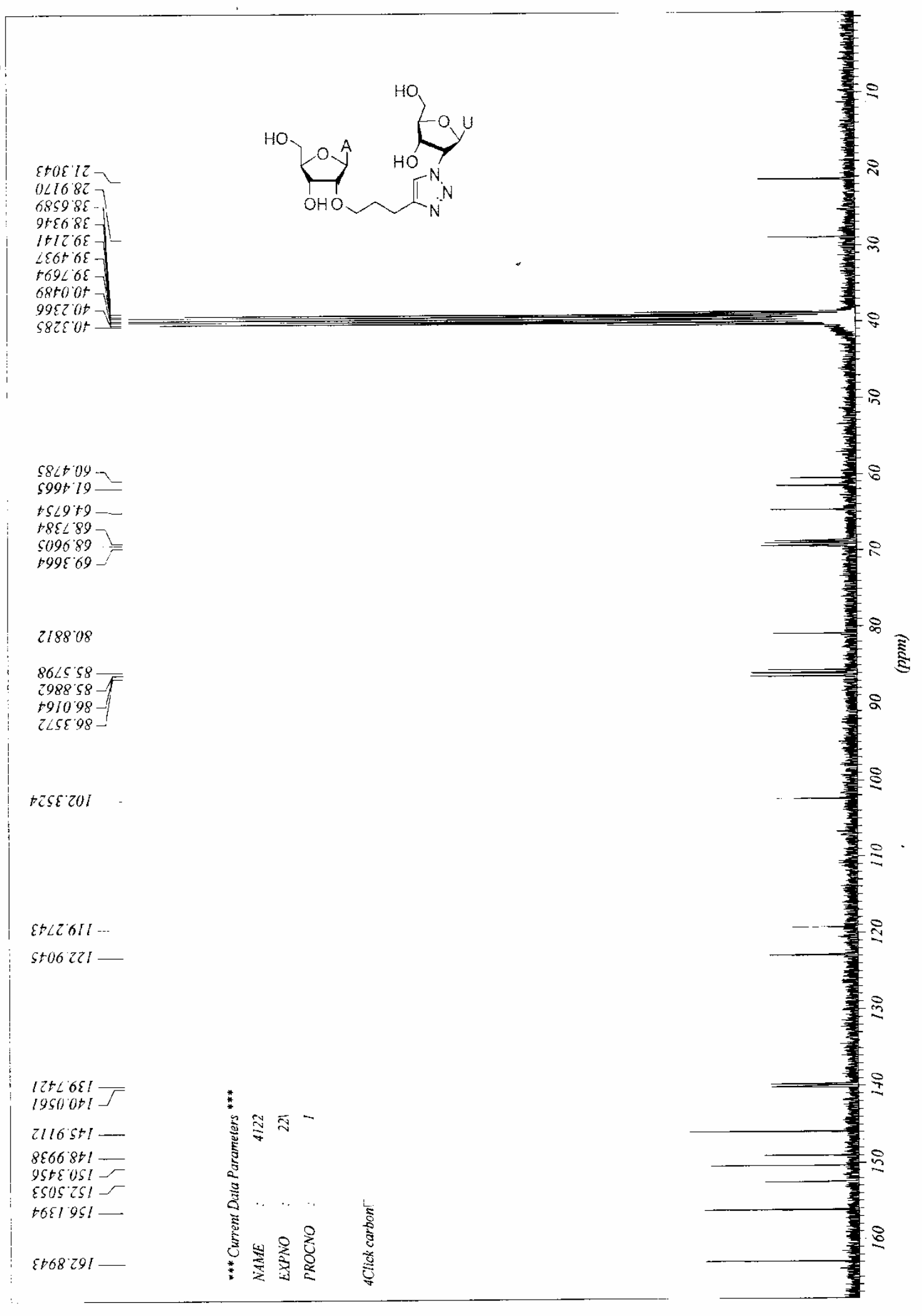




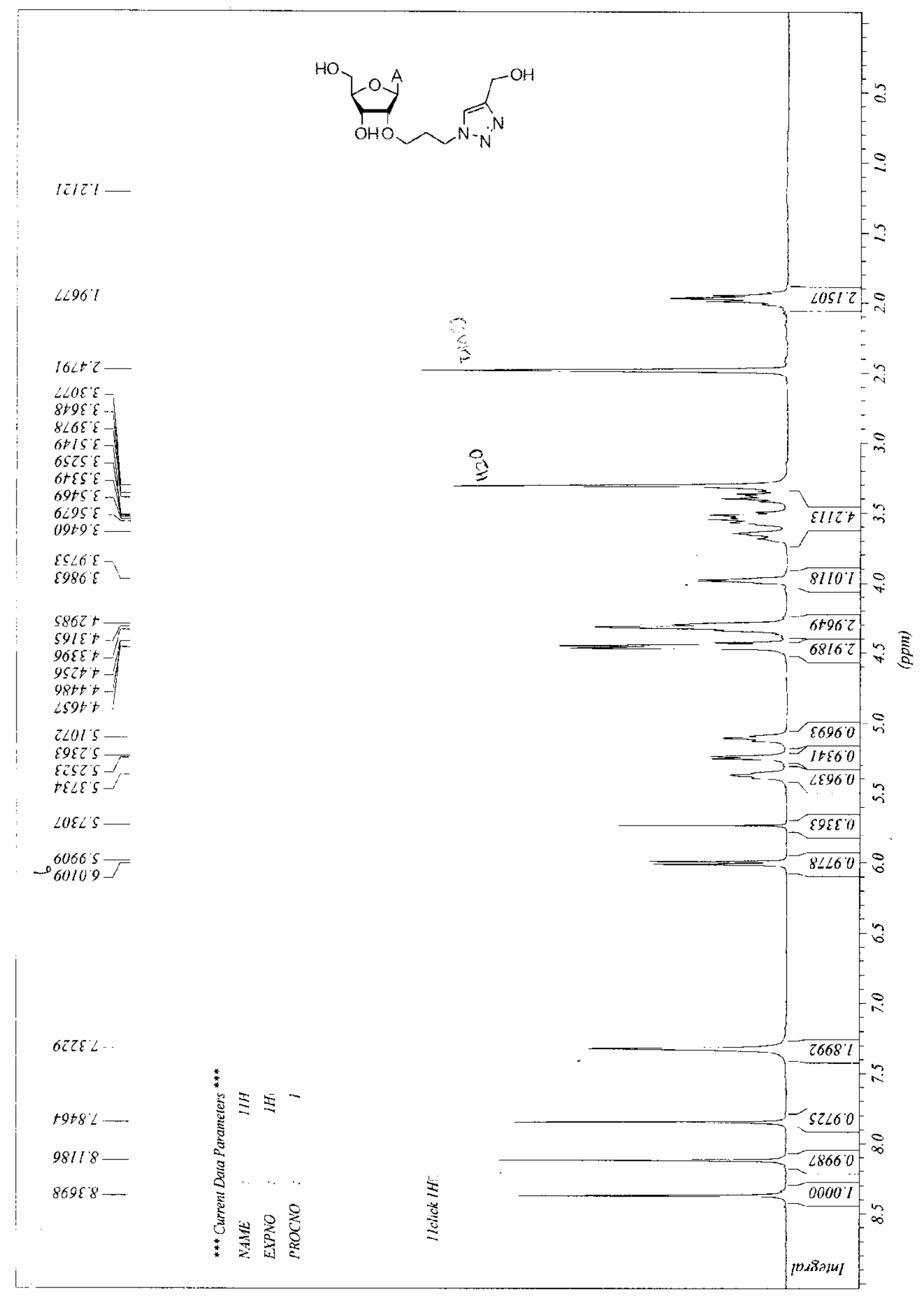




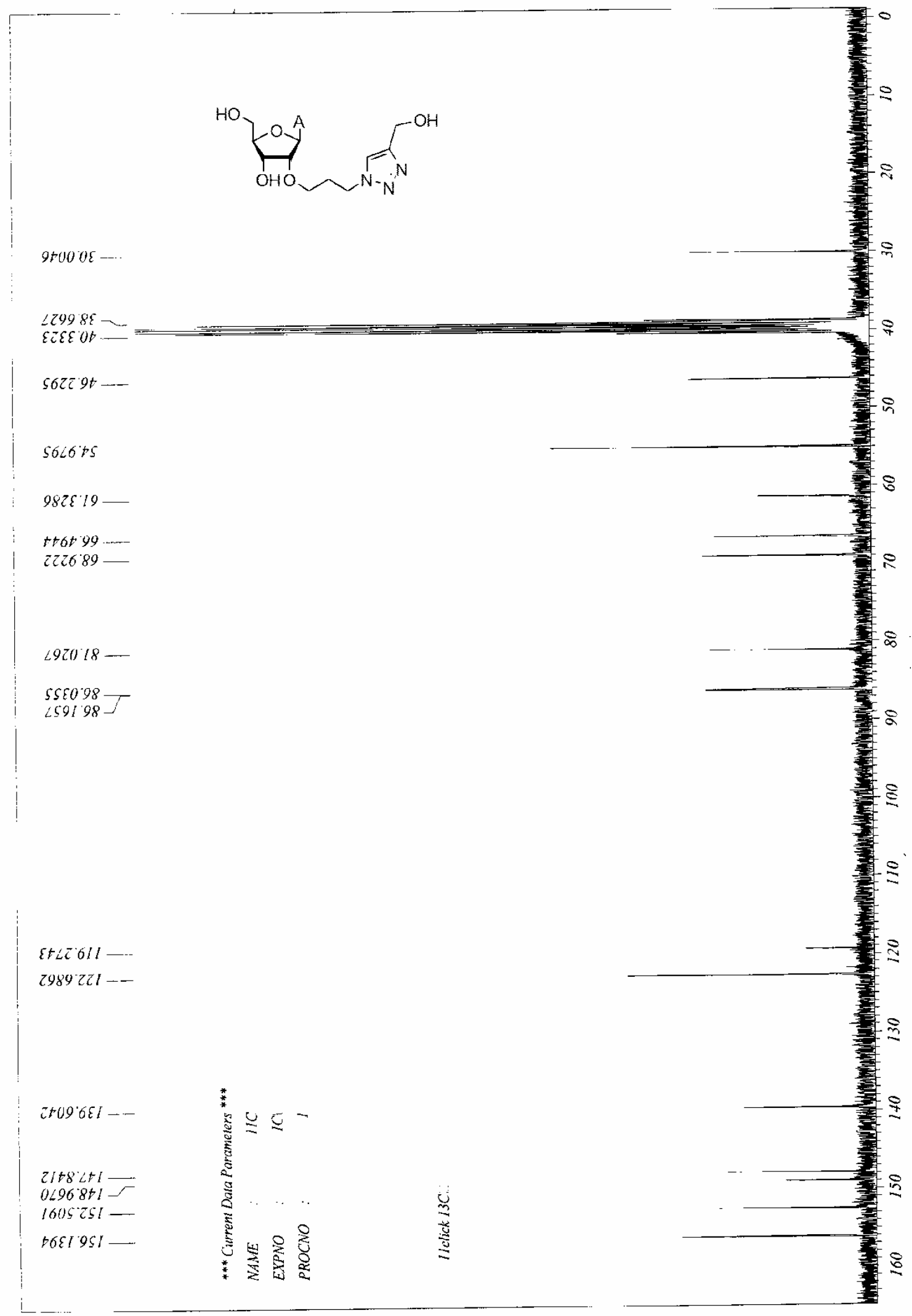


$0861 \%$

zट⿱中⿰㇀丶㇀

cltt $1=$

59657

$58+6 \%$

$4627 \mathrm{Z}$

19812

$199+2$

$9605 \mathrm{z}$

$987 S^{\circ} \mathrm{C}$

$2 \angle+S \cdot 2$

$9869 \% \ldots$

9toc $\varepsilon$

$58+52-$

$8+\pi 5 \varepsilon=$

IE\&S:

SSSS $\mathcal{S}$

$\operatorname{sit} \theta \dot{\varepsilon}$

II $\angle 6 \mathcal{E}-T$

$66 \angle 6 \varepsilon$

s992'

$89 \angle 2 \%-$

$616 c^{\circ}+$

$\angle 660^{\circ}+$

$\angle E 8)^{\circ}{ }^{\circ}$

$8+\angle Z S \cdots=$

$5 \angle 8 Z 5{ }^{\prime}$

61009.

19109
$\mathrm{HO}$

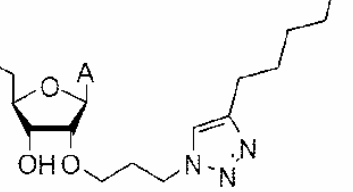

$\mathrm{OHO} \sim \mathrm{N}-\mathrm{N}$

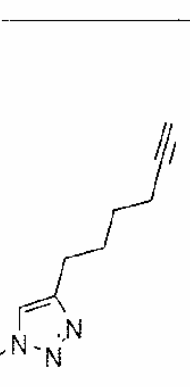

2

$+2$

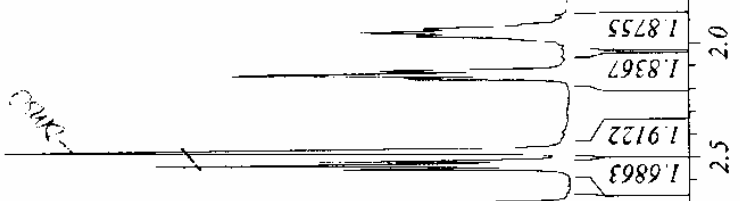

96080
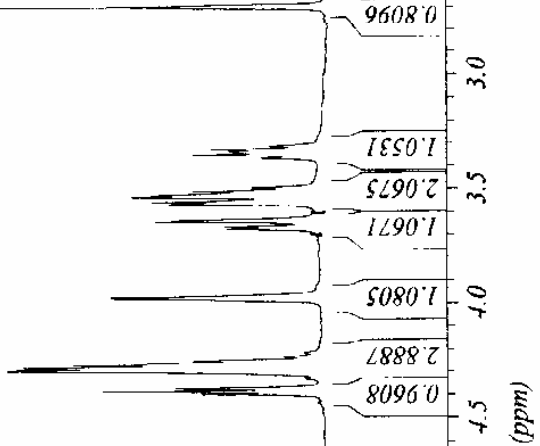

\section{*}

$2166 \mathrm{~L}$

16258

$6+8+8$

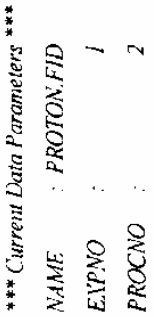

ฟั้

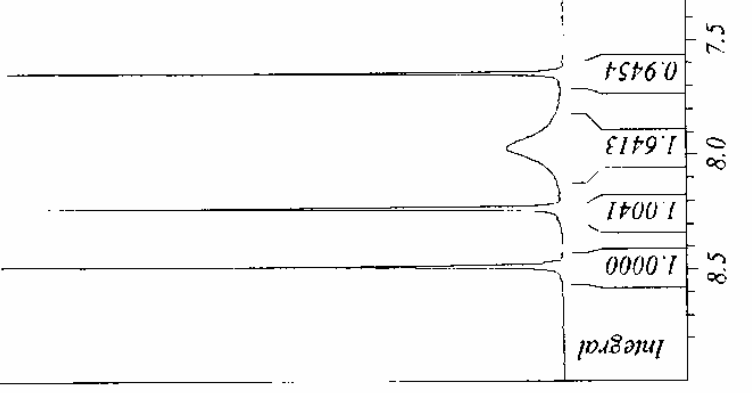




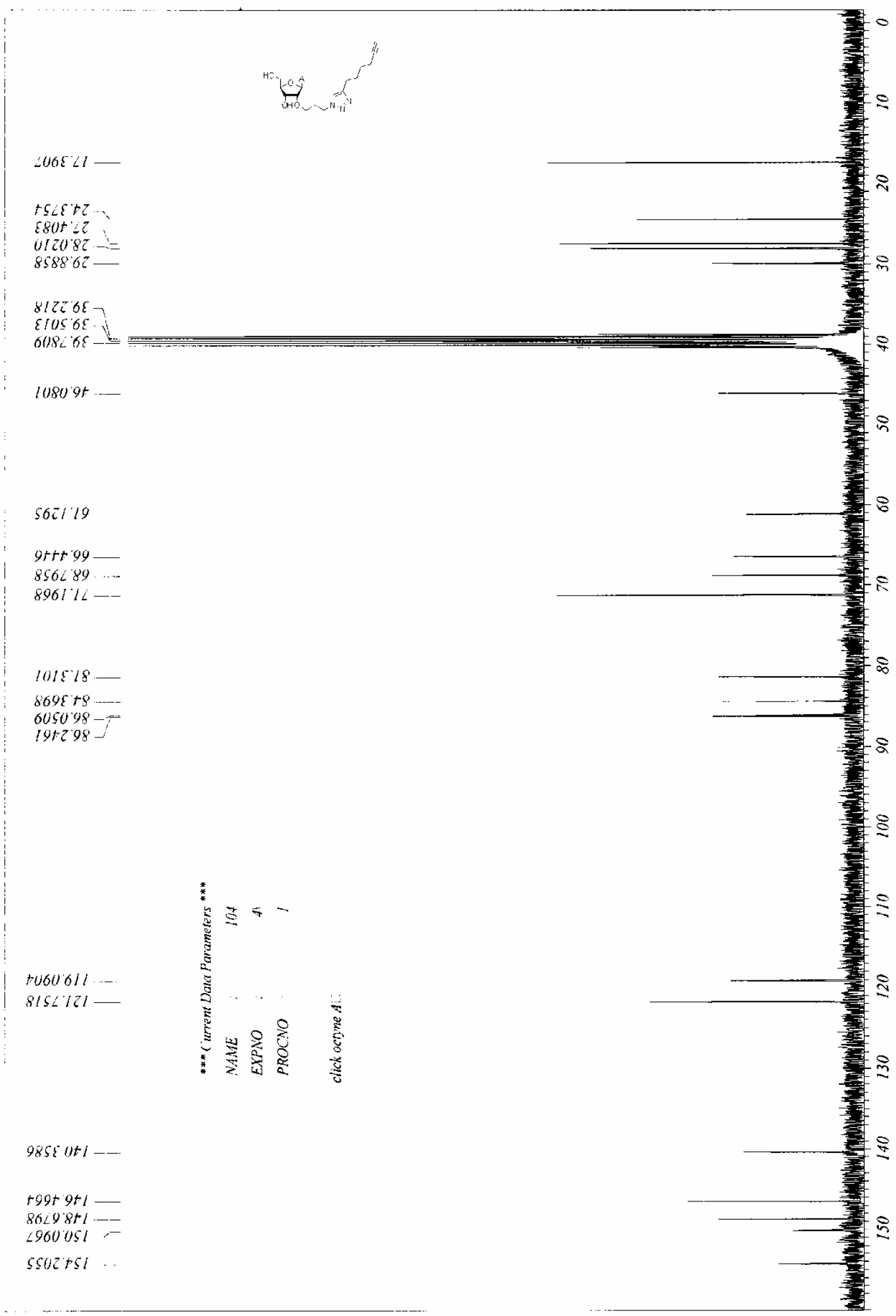




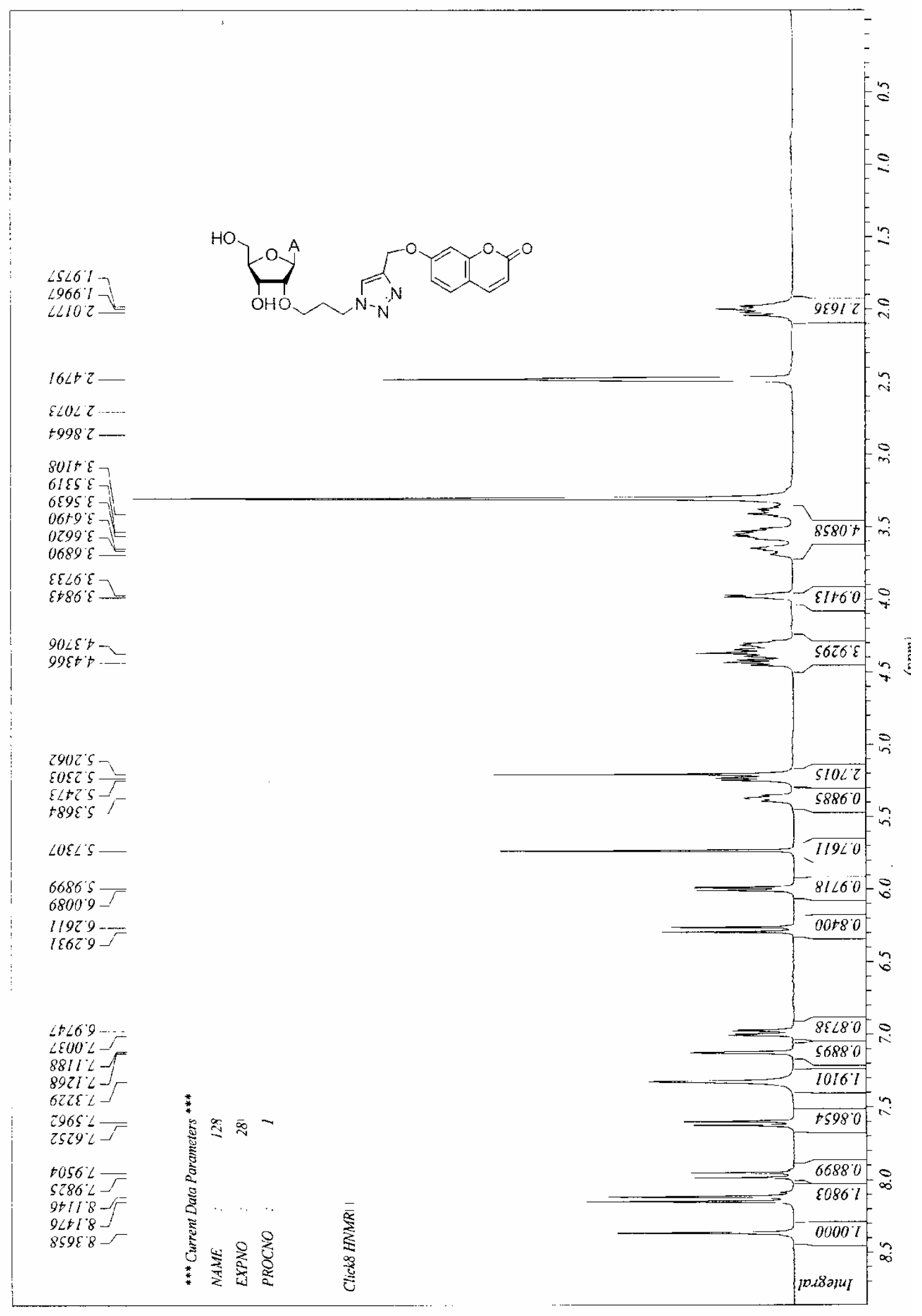




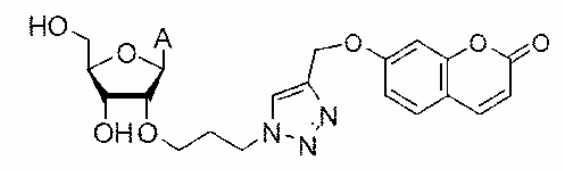

$5 \varepsilon 686 \overline{6}-$

6zIS.9t--

$0088^{\circ}+5-$

9508197

959919\llcorner

$+56+99$

$092689 \ldots$

219078

605098

$99+198 \longrightarrow$

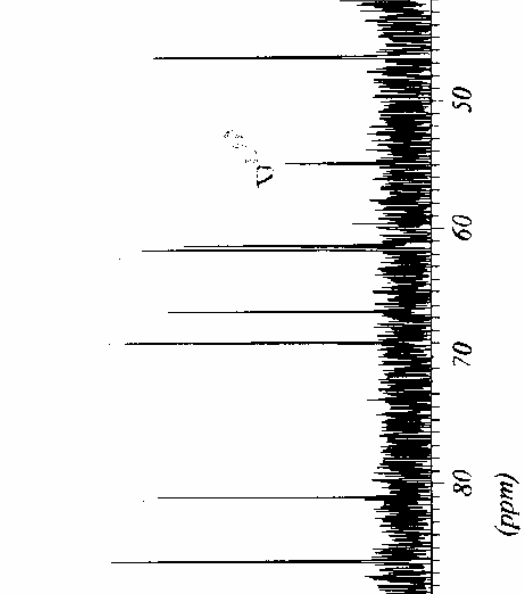

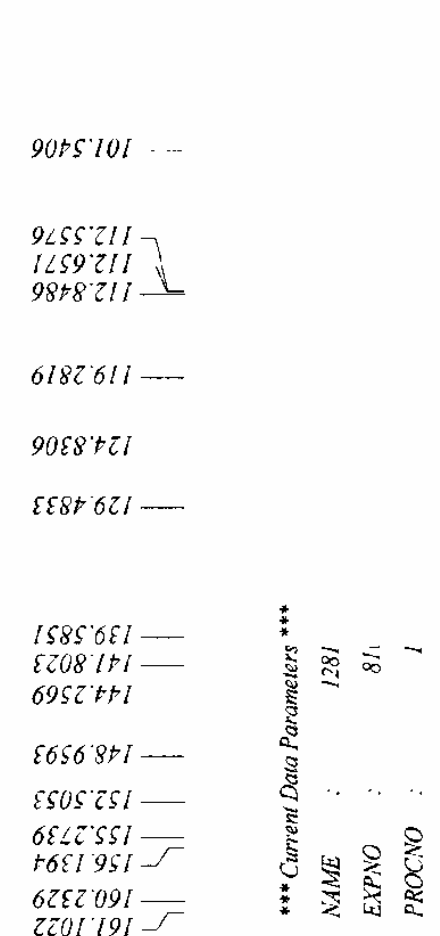

足

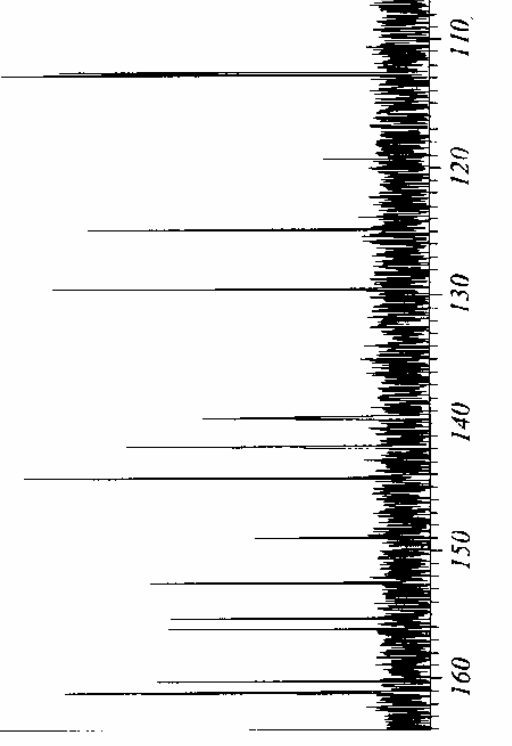




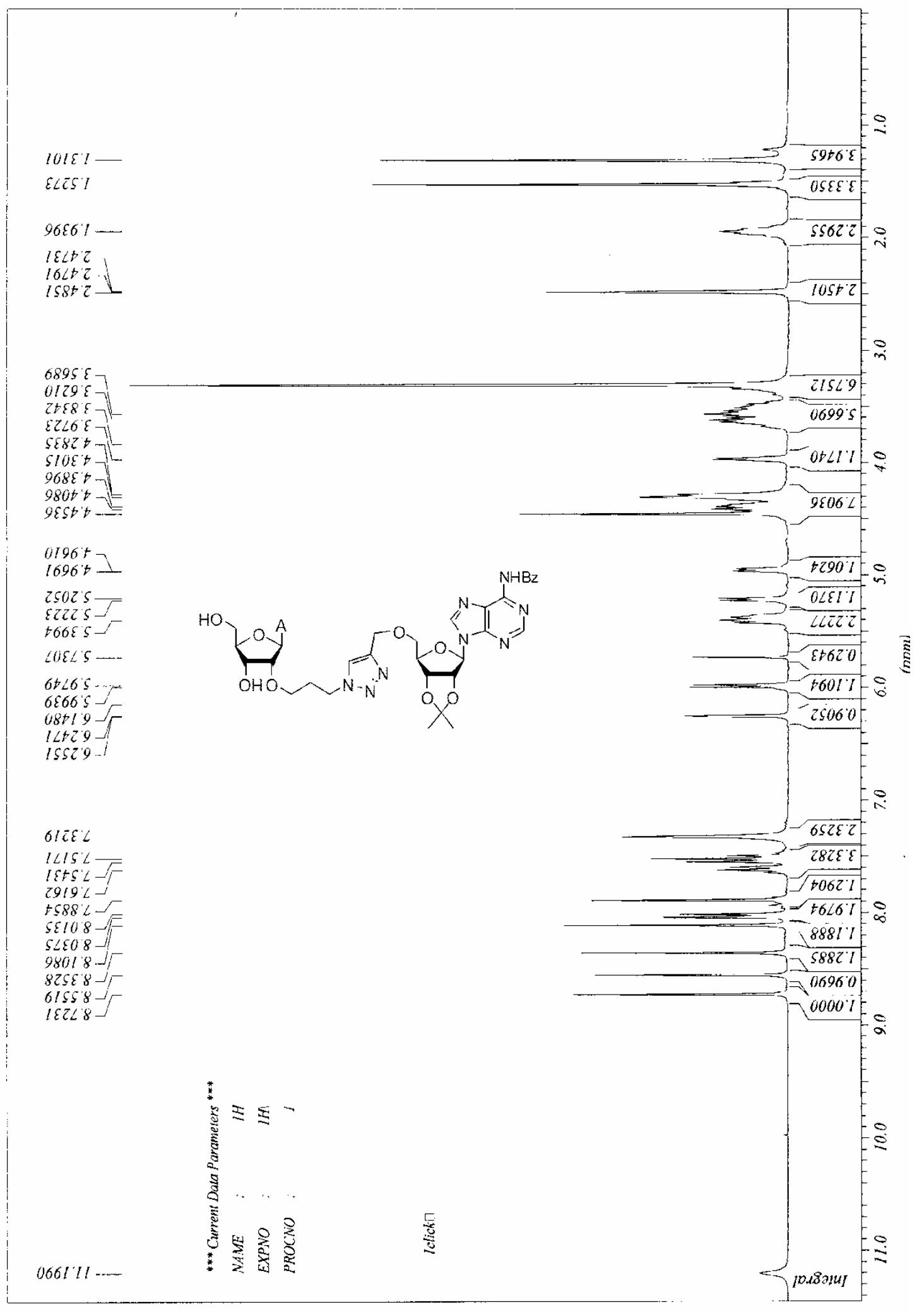




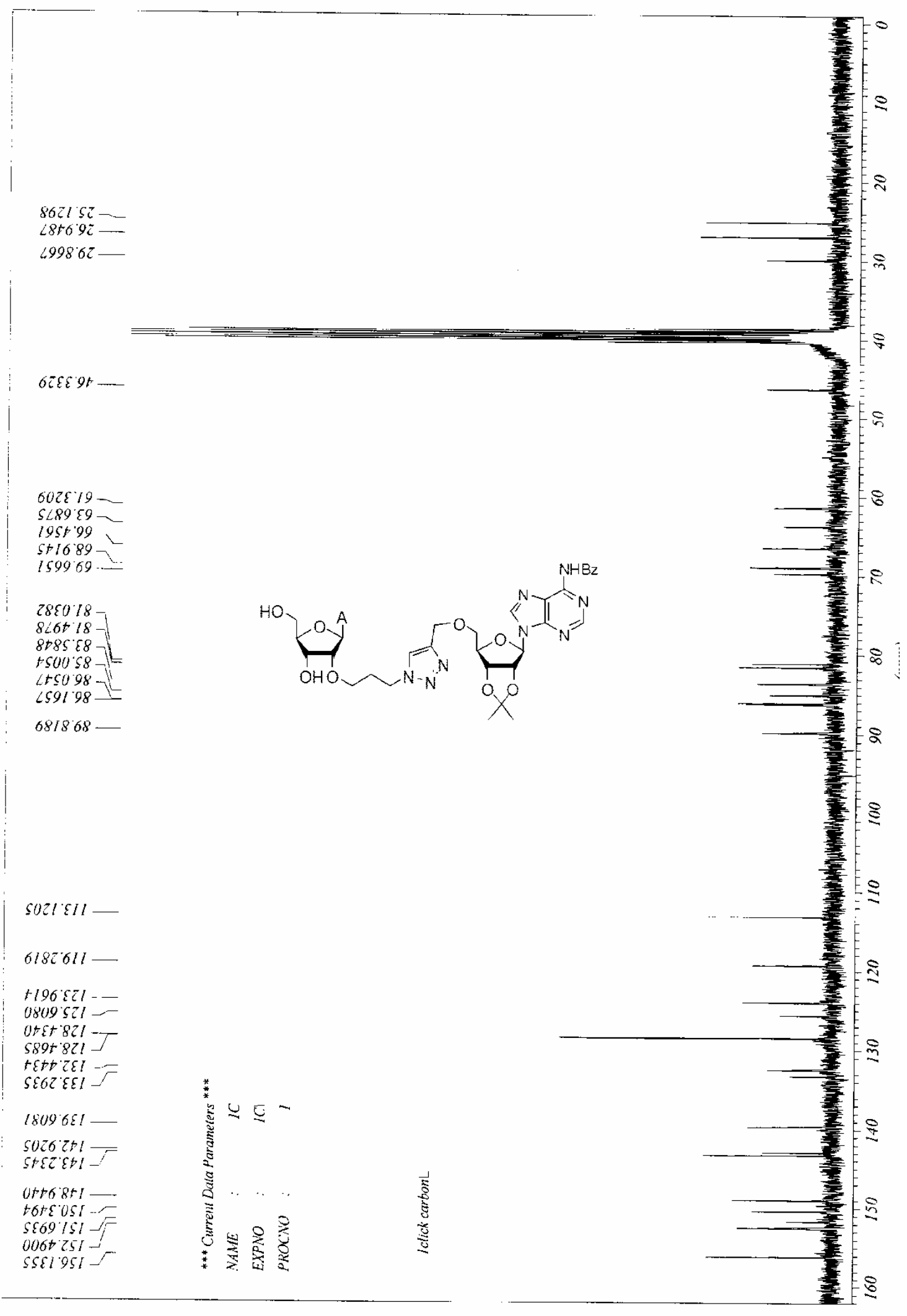




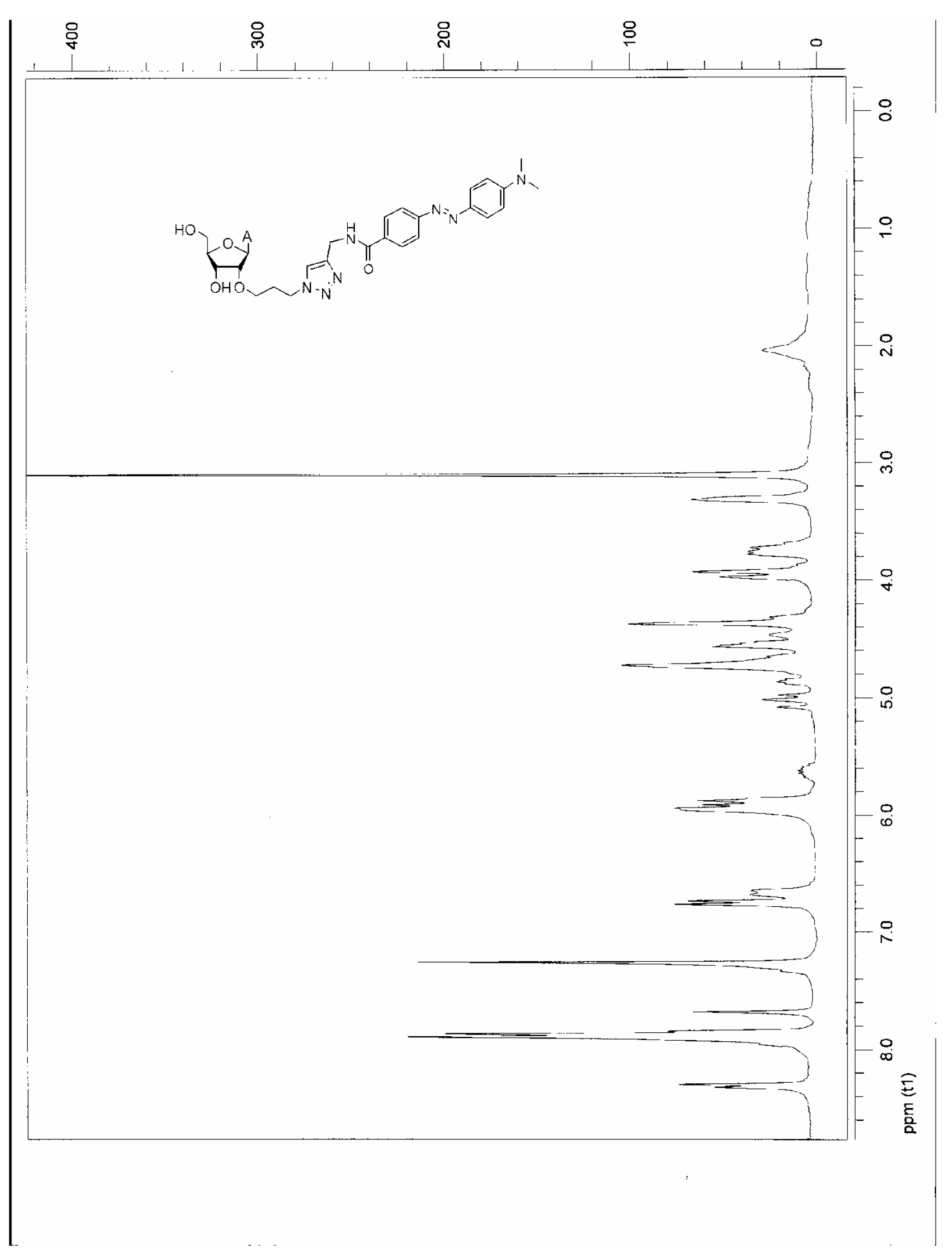




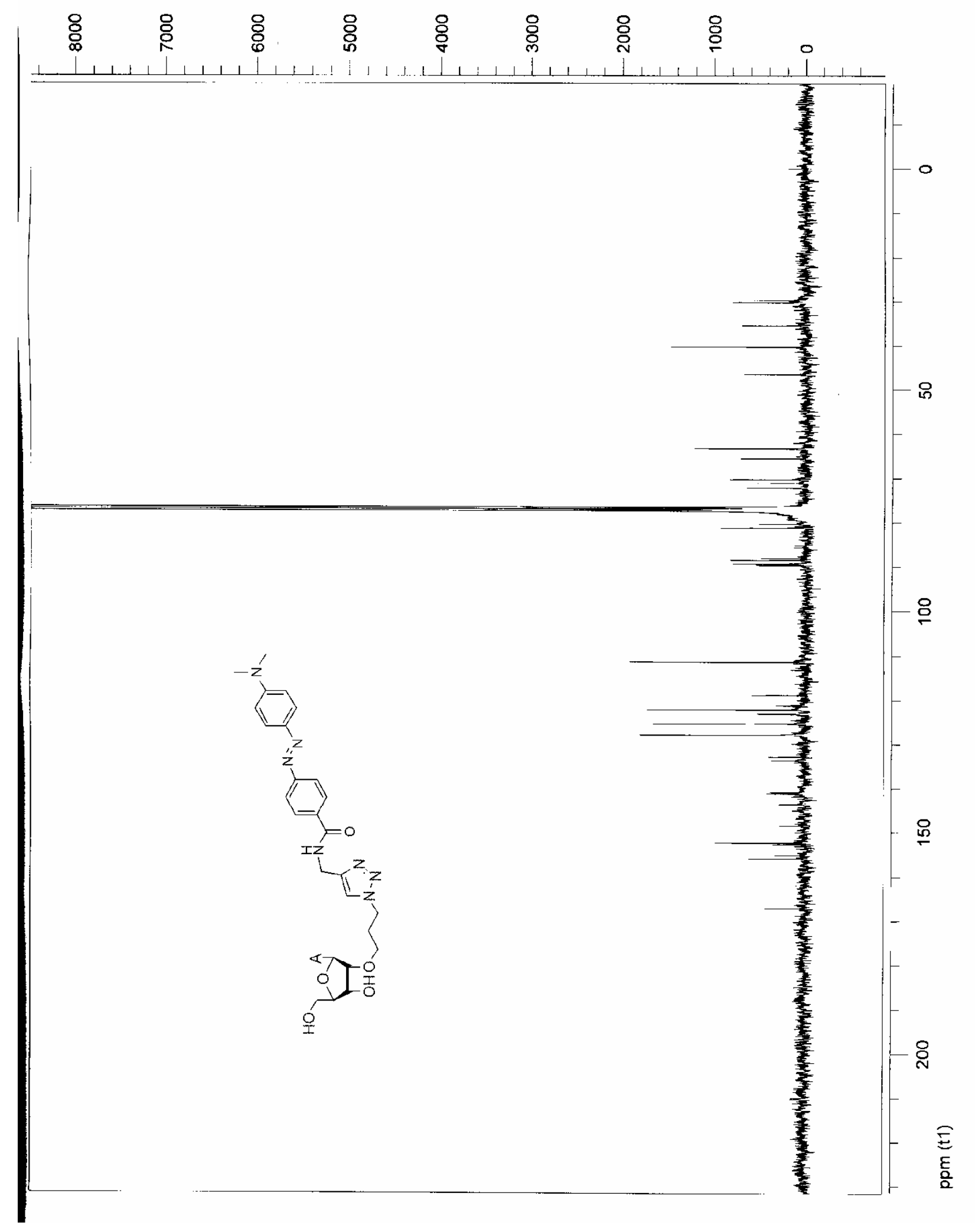




\section{References}

1 Yao, L.; Smith, B.T.; Aubé, J. J. Org. Chem. 2004, 69, 1720.

${ }^{2}$ Shiozaki, M.; Mochizuki, T.; Hanzawa, H.; Haruyama, H. Carbohydr. Res. 1996, $288,99$.

3 Jahn, W. Chem. Ber. 1965, 98, 1705.

${ }^{4}$ Wnuk, S. F.; Chowdhury, S. M.; Garcia, P. I.; Robins, M. J. J. Org. Chem. 2002, 67, 1816.

${ }^{5}$ Bushmakina, N. G.; Misharin, A. Yu Synthesis 1986, 966.

6 a) Ishikawa, T.; Mizutani, A.; Miwa, C.; Oku, Y.; Komano, N.; Takami, A.; Watanabe, T. Heterocycles 1997, 45, 2261.b) Kosiova, I.; Kovackova, S.; Kois, P. Tetrahedron 2006, 63, 312.

${ }^{7}$ Maggiora, L. L.; Smith, C. W.; Zhang, Z. Y. J. Med. Chem. 1992, 35, 3727

8 a) Yano, J.; Kan, L. S.; Ts'o, P. O. P. Biochim. Biophys. Acta 1980, 629, 178. b) Hyde, R. M.; Broom, A. D.; Buckheit, R. W., Jr. J. Med. Chem. 2003, 46, 1878.

${ }^{9}$ Campos-Toimil, M.; Orallo, F.; Santana, L.; Uriarte, E. Bioorg. Med. Chem. Lett. 2002, 12, 783.

${ }^{10}$ Mitra, A. K.; De, A.; Karchaudhuri, N. Ind. J. Chem., Sect B. 2000, 39, 311.

${ }^{11}$ Heikkilae, J.; Bjoerkman, S.; Oeberg, B.; Chattopadhyaya, J. Acta Chem. Scand. B. 1982, 36, 715. 